University of Louisville

ThinkIR: The University of Louisville's Institutional Repository

Electronic Theses and Dissertations

$8-2020$

\title{
Costs and benefits of HIPV-mediated eavesdropping under field conditions.
}

Grace Ellin Freundlich

University of Louisville

Follow this and additional works at: https://ir.library.louisville.edu/etd

Part of the Ecology and Evolutionary Biology Commons

\section{Recommended Citation}

Freundlich, Grace Ellin, "Costs and benefits of HIPV-mediated eavesdropping under field conditions." (2020). Electronic Theses and Dissertations. Paper 3483.

https://doi.org/10.18297/etd/3483

This Doctoral Dissertation is brought to you for free and open access by ThinkIR: The University of Louisville's Institutional Repository. It has been accepted for inclusion in Electronic Theses and Dissertations by an authorized administrator of ThinkIR: The University of Louisville's Institutional Repository. This title appears here courtesy of the author, who has retained all other copyrights. For more information, please contact thinkir@louisville.edu. 
COSTS AND BENEFITS OF HIPV-MEDIATED EAVESDROPPING UNDER FIELD CONDITIONS

\author{
By \\ Grace Ellin Freundlich \\ B.S. Dickinson College, 2014 \\ A Dissertation \\ Submitted to the Faculty of the \\ College of Arts and Sciences of the University of Louisville \\ in Partial Fulfillment of the Requirements \\ for the Degree of \\ Doctor of Philosophy in Biology \\ Department of Biology \\ University of Louisville \\ Louisville, Kentucky
}

August 2020 



\title{
COSTS AND BENEFITS OF HIPV-MEDIATED EAVESDROPPING UNDER FIELD CONDITIONS
}

\author{
By
}

Grace E. Freundlich

B.S. Dickinson College, 2014

A Dissertation Approved on

June 24,2020

By the Following Dissertation Committee

Dissertation Director

Dr. Sarah Emery

Dr. Steve Yanoviak

Dr. Mark Running

Dr. David Schultz

Dr. Robert Schaeffer 


\section{ACKNOWLEDGEMENTS}

I would've never completed this dissertation without the unwavering support of my friends, family, and my partner Chris Bierman. This has been the hardest thing I've ever done, and they tirelessly motivated me to finish with their unwavering support. Second, I would like to thank my dissertation committee for their guidance and support throughout this process. Additionally, I would like to thank Dr. Chris Frost for initially recruiting me into the Biology department and for supporting my early efforts on an NSF grant. Next, I would like to thank the University of Louisville College of Arts and Sciences for small research grants and the Blackacre Conservancy for allowing me to conduct my research in the community garden. Finally, I would like to thank my advisor Dr. Sarah Emery for helping me finish my program and for helping me reach my full potential as a scientist. She has taught me what it means to be a great mentor and scientist and I will always be grateful to her. 


\section{ABSTRACT \\ INVESTIGATING THE COSTS OF EAVESDROPPING UNDER FIELD CONDITIONS}

Grace Freundlich

June 24,2020

Eavesdropping is defined as the act of an organism taking advantage of a cue that was not originally intended for them for their own gain. Plant-mediated eavesdropping is a widely documented phenomenon and eavesdropping cues range from oviposition cues to airborne volatile compounds. Eavesdropping is predicted to be widespread because eavesdropping confers a fitness advantage. In plants, eavesdropping on herbivore-induced plant volatiles (HIPVs) often results in enhanced resistance profiles upon herbivory. As a result of this enhanced resistance, it is predicted that eavesdropping alone incurs minor costs due to resource shifts towards inducible defense responses. Although HIPV-mediated eavesdropping is widely documented, few studies have investigated ecological factors that impact costs of eavesdropping, especially under field conditions.

In this dissertation, I investigated factors that impact a plant's utilization of volatile cues, and how eavesdropping affects growth and defense profiles in eavesdropping plants. In the second chapter, I studied how exposure to a ubiquitous 
plant derived compound, the green-leaf volatile cis-3-hexenyl acetate (z3HAC), affected Phaseolus lunatus (Lima bean) and Capsicum annuum var. Cayenne (Cayenne pepper). Although they were exposed to identical treatments, exposed pepper plants experienced vegetative and reproductive costs while lima bean had increased growth from volatile exposure alone. In the subsequent field season, I found that duration of exposure to $z 3 \mathrm{HAC}$ significantly impacted overall growth in lima bean. To determine if volatile blends differentially impact growth and defense profiles, I manipulated neighboring herbivore and plant identity to assess costs of eavesdropping. While neighboring plant identity had some effects on overall biomass, herbivore identity impacted resource allocation towards indirect defenses. To determine why plant-plant eavesdropping is observed, I used seeds collected from the experiment in chapter 4 and grew them in the next field season. Counter to our predictions, maternal eavesdropping had no effect on plant performance such as growth and defense. These results indicate that the costs and benefits of eavesdropping under field conditions are extremely nuanced and impacted by environmental variation, such as herbivore pressure and abiotic stress. 


\section{TABLE OF CONTENTS}

ACKNOWLEDGMENTS

CHAPTER III: Growth-defense tradeoffs of eavesdropping are affected by duration of exposure to a green leaf volatile .......................................................................... 26

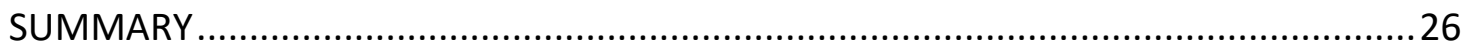

INTRODUCTION

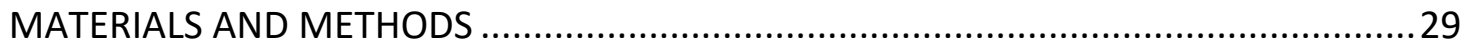

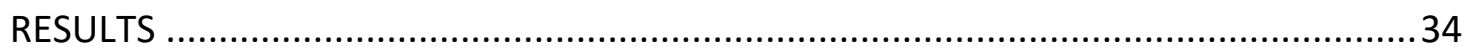

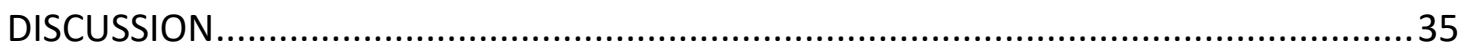

CHAPTER IV: Herbivore identity has indirect fitness effects via herbivore-induced plant volatiles in $P$. lunatus under field conditions

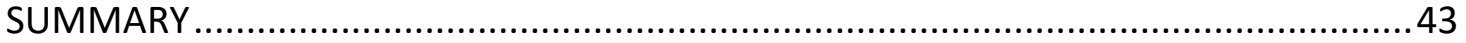

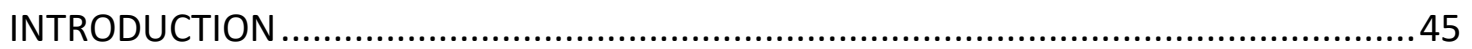

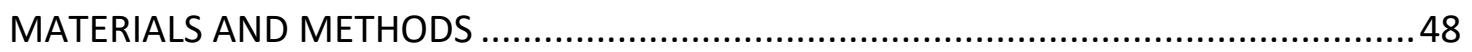

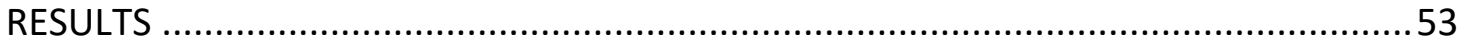

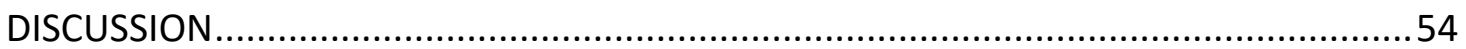


CHAPTER V: Maternal eavesdropping has no effects on progeny performance under field conditions

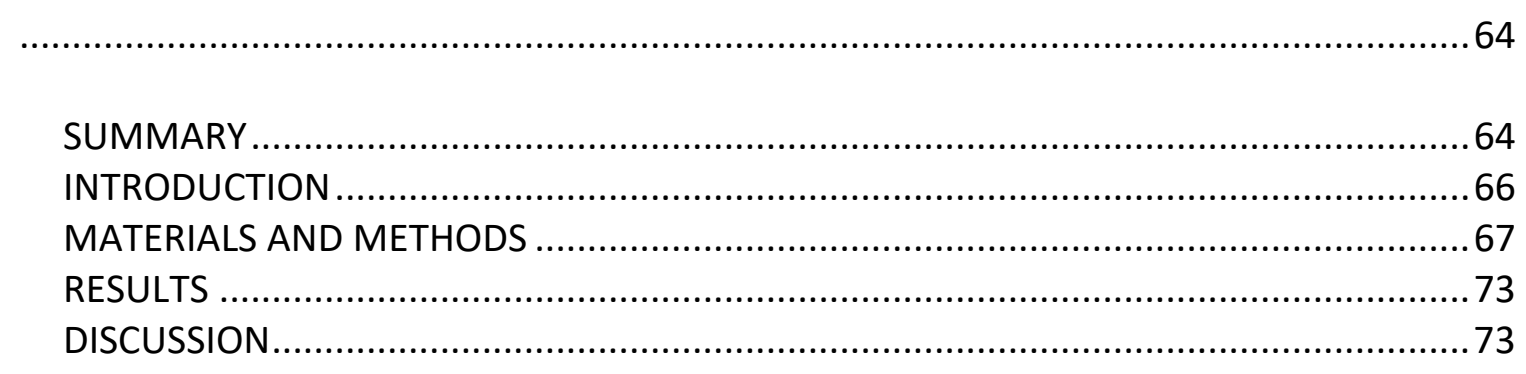

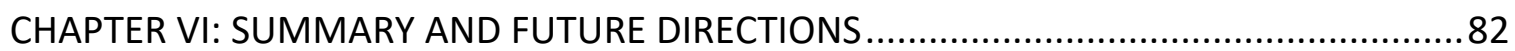

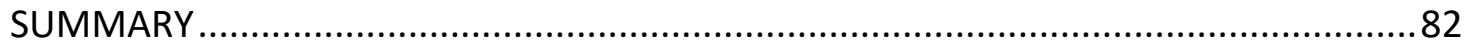

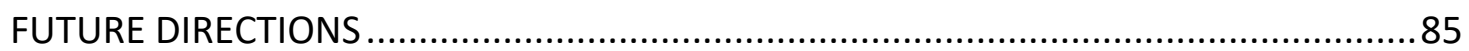

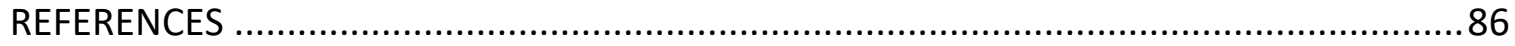

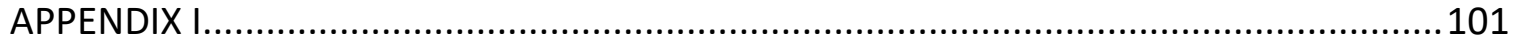

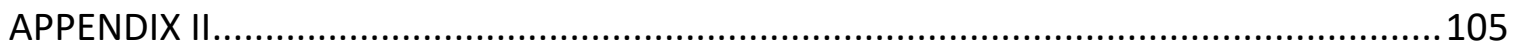

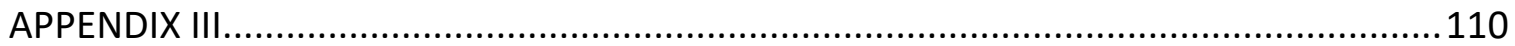

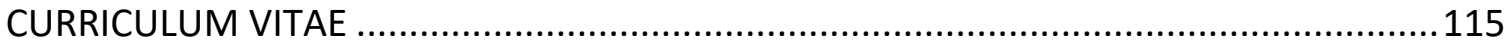




\section{LIST OF TABLES}

TABLE

1. Summary statistics for growth and defense metrics for $P$. lunatus ...........................38

2. Growth effects for eavesdropping on HIPVs overtime .......................................57

3. Growth effects for eavesdropping on HIPVs at each timepoint ..............................58

4. Two-way ANOVA results for F1 early development and performance.......................76

5. One-way MANOVA results for assessing parental effects on in-field reproductive output

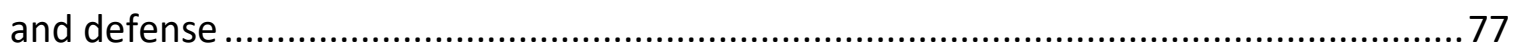




\section{LIST OF FIGURES}

FIGURE

PAGE

1. Height measurements and leaf counts for Phaseolus lunatus (lima bean) and Capsicum annuum (pepper) grown in a common garden field experiment. 21

2. Biomass measurements of field-grown Capsicum annuum (pepper) plants.

3. Total flower production in (a) Phaseolus lunatus (lima bean) and (b) Capsicum annuum (pepper)

4. Fruit and seed production in Capsicum annuum (pepper) plants grown in a common garden experiment treated with a persistent application of the green leaf volatile cis-3hexenyl acetate (z3HAC). 24

5. Herbivore damage on Capsicum annuum (pepper) and Phaseolus lunatus (lima bean) plants in a common garden field experiment .25

6. Volatile administration setup .39

7. Volatile duration differentially affects biomass in $P$. lunatus. .40

8. Volatile exposure type does not affect reproductive structures and output in P. lunatus.

9. $z 3 H A C$ administration does not impact cyanide induction or chewing herbivore resistance in the field

10. Experimental setup within the field site for chapter 4 .59

11. Herbivore effects on reproductive output in eavesdropping plants 60

12. Eavesdropping on neighboring HIPVs has no effect on overall biomass........ 61 
13. Eavesdropping effects on direct and indirect defense ......................................62

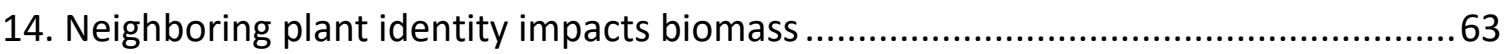

15. F1 germination effects of parental eavesdropping ............................................. 78

16. F1 phenology effects of parental eavesdropping......................................................79

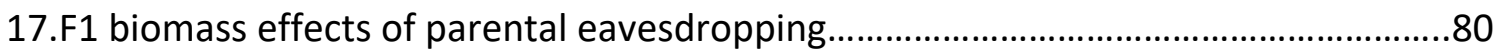

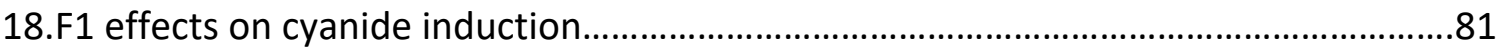




\section{CHAPTER I}

\section{INTRODUCTION}

Almost any defense mechanism, whether it is chemically [1] or physically $[2,3]$ mediated, incurs costs as a result of energetic tradeoffs between growth and defense [4]. As a result of their sessile nature, plants must account for environmental variation to optimize defense allocation. Induction studies with phytohormones, such as jasmonic acid and its conjugates, verify that costs occur with initiation of defense since induction often results in enhanced herbivore resistance at the cost of reduced growth [5-7]. Costs of defense are especially apparent under varying biotic and abiotic factors, such as population level differences across environmental gradients [8] and herbivore pressure. The ability for a plant to be plastic allows for optimized resource allocation to prioritize fitness and minimize costs of defense [9]. The defense horizon, or underlying constitutive levels of defense, is significantly impacted by herbivore presence and pressure. As a result, baseline levels of defense are only present when necessary and vary in inducibility $[7,10,11]$. Environmental variation and costs determine if defenses are constitutively maintained or induced $[12,13]$. For example, in the absence of herbivores, Palmer et al found that Acacia plants under long-term herbivore exclusion had reduced spine counts and increased reproductive output relative to controls [14]. Similarly, energy previously utilized for chemical defenses was diverted towards 
reproductive growth in grasses when herbivores were excluded over multiple generations [15]. These dramatic shifts in resource allocation away from defense indicate various underlying mechanisms for minimizing costs and adapting to localized stress in real time.

Plant-plant eavesdropping is a cost saving strategy that allows a plant to anticipate herbivory before it occurs. Plants eavesdrop on various types of environmental cues that are associated with herbivory $[16,17]$. These cues range in identity and include leaf vibrations caused by herbivores [18], compounds secreted from insect oviposition $[19,20]$, mucus from snail trails [21], and herbivore-induced plant volatiles $[17,22]$ released from herbivore-damaged leaves, stems, and roots. The ability to determine an accurate signal within the environment is pivotal for optimizing defense and reducing the risk of responding to an unreliable cue [9]. Within plants, most studies have focused primarily on the impact of herbivore-induced plant volatiles (HIPVs) [23]. HIPVs vary in size and structural complexity, ranging from green-leaf volatiles (GLVs) [24] to sesquiterpenoids [25-27].

In plant-herbivore interactions, herbivore-induced plant volatile (HIPV)-mediated eavesdropping is widely observed because HIPVs serve as reliable indicators of herbivory [26]. HIPVs are ubiquitously released simultaneously upon mechanical [28] and herbivore-induced damage [29], accurately depict herbivore feeding guild [30], and identity of the emitting plant [31, 32]. HIPV-mediated eavesdropping has been documented across the plant kingdom. While most papers have documented eavesdropping in agricultural systems [33-39], trees shrubs [40-42], and non-vascular 
plants [43], we know very little about the conditions in which eavesdropping plants use HIPVs. Within this dissertation, I wanted to elucidate ecological factors that may impact why plants utilize volatile cues and begin to understand why eavesdropping is observed within the plant kingdom.

\section{Organization of Dissertation}

In this dissertation, I separately tested individual ecological factors that influence when a plant may utilize a volatile cue before experiencing herbivory. Within this work, I utilized Phaseolus lunatus and Capsicum annuum to assess the ecological costs and benefits of eavesdropping within common garden experiments at Blackacre Conservancy in Louisville, KY. I assessed proximate measures of fitness, such as overall growth and reproductive output, as well as in-field herbivore resistance measures and direct defense quantification to elucidate factors that allow eavesdropping itself to become a selective pressure.

In my second chapter, I compared Phaseolus lunatus and Capsicum annuum growth and defense profiles to determine if different plant species respond similarly to a ubiquitous GLV cis-3-hexenyl acetate (z3HAC). Both species were grown under field identical conditions and exposed to a continuous duration of 10 ng/hour for 140 days. While $C$. annuum experienced reduced growth, $P$. lunatus invested more in overall growth and was more resistant to chewing herbivores under identical field conditions.

The third chapter investigated how duration of $z 3 \mathrm{HAC}$ affected the costs of eavesdropping in $P$. lunatus. Since volatile duration can act as a contextual cue for herbivore pressure, I predicted that increased duration would result in increased costs 
relative to controls. For this study, I exposed $P$. lunatus to three separate duration periods of $z 3 \mathrm{HAC}$ and assessed changes in growth and in-field defense. These treatments consisted of a continuous exposure, a repeated 24-hour weekly exposure (pulsed) and a one-week continuous exposure period (transient) during the growing season. While in-field defense metrics, such as in-field herbivory and cyanide induction did not differ across treatments, I found that pulsed and continuous exposure to z3HAC resulted in increased stem and total biomass relative to control and transient treatments.

Since I found alterations in biomass from $z 3 \mathrm{HAC}$ exposure alone, in the next chapter, I wanted to determine if volatile specificity would impact costs of eavesdropping via manipulating neighboring herbivore identity. In chapter 4, I experimentally manipulated neighboring herbivore identity as well as emitting cultivar identity to address this question. Overall, I found that eavesdropping plants respond differently to herbivore-specific volatiles for defense while herbivore and neighboring plant identity have relatively little effect on overall growth. In this case, I found interactive effects between eavesdropping plant identity and neighboring herbivore identity for indirect defense. While direct defense metrics were unaffected by eavesdropping, exposure to volatiles generated by Anticarsia gemmatalis resulted in decreased extra-floral nectary production.

To begin to explain why eavesdropping is a conserved trait, I collected and grew seeds from the experiment in chapter 4 to determine if maternal eavesdropping impacts next-generation performance, and if these effects are comparable to maternal effects 
from herbivory. In chapter 5, I found that maternal eavesdropping had no effects on chemical investment in the next generation and no impacts on growth. While transgenerational effects were not observed, it is important to note that environmental conditions may influence when transgenerational effects are observed. Therefore, these tradeoffs from eavesdropping may fall along a spectrum of inducibility that suggests that eavesdropping results in moderate inducible responses that incur costs.

The final chapter summarizes the main conclusions of the dissertation and provides avenues for future research questions to gain a greater understanding of plantmediated eavesdropping and how this knowledge can be applied within agricultural settings. 


\title{
CHAPTER II
}

\section{EXPOSURE TO A UBIQUITOUS GLV RESULTS IN SPECIES-SPECIFIC COSTS}

\begin{abstract}
Summary
Herbivore-induced plant volatiles (HIPVs) may cue eavesdropping neighboring plants to 'prime' defenses prior to experiencing herbivory. Defense priming is an inducible phenomenon that-like any inducible phenomenon-should incur fitness costs. Such costs have not been documented under field conditions. I predicted that exposure to an HIPV would reduce plant growth and reproduction. I treated lima bean (Phaseolus lunatus) and pepper (Capsicum annuum) plants in a common field experiment with a persistent, low-dose ( $\sim 10 \mathrm{ng} / \mathrm{hour}$ ) of cis-3-hexenyl acetate (z3HAC), an HIPV that alone can prime plants. Whereas $33 \mathrm{HAC}$-treated pepper plants had less biomass and produced fewer flowers and fruits than did controls, z3HAC-treated lima bean plants were larger, produced more leaves and flowers, and had less natural herbivore damage than did controls. Thus, a single volatile priming cue induces divergent, species-specific effects on growth, reproduction, and defense. These results suggest that plant life history may influence how plants respond to volatile indicators of herbivory.
\end{abstract}




\section{$\underline{\text { Introduction }}$}

Production and utilization of airborne chemical cues is prevalent within the plant kingdom. Plants depend on airborne chemical signaling for pollination [44], indirect defense [45], protection from pathogens [46], and herbivore resistance [47]. Volatile communication is also pivotal for plant-plant signaling, and selection for such signaling depends on honest cues that reliably confer ecologically relevant information. For example, herbivory is a fundamental ecological interaction that impacts plant fitness, and many plants induce the production and emission of volatile compounds in response to herbivore damage [48]. Such herbivore-induced plant volatiles (HIPVs) then become potentially reliable cues around which plant-plant eavesdropping could be evolutionarily adaptive. Undamaged plants (or parts of the same plant $[49,50]$ ) eavesdropping on HIPVs from a plant experiencing herbivory may directly trigger stress responses [51-53], or alternatively prime responses for future potential herbivory [17].

HIPV-mediated priming appears to be widespread across the plant kingdom. HIPVs have demonstrated priming ability in corn [54, 55], tomato [56], poplar [47, 49], blueberry [57] and lima bean [58, 59]. HIPVs can be diverse and taxon-specific [60, 61], but are often comprised of monoterpenes, sesquiterpenes, benzenoids and green leaf volatiles (GLVs) $[62,63]$. In contrast to volatile terpenes and benzenoids $[60,64]$, GLVs are immediately released into the airspace whenever leaves are mechanically damaged [65] serving as reliable indicators of wounding. GLV exposure alters gene expression profiles related to specialized metabolite production and accumulated secondary metabolite precursors in preparation for inducing resistance [66]. For example, the GLV 
cis-3-hexenyl acetate (z3HAC) induces transcriptional changes in poplar [47] and maize [63] that prime oxylipin signaling and induced resistance. Among the GLVs, z3HAC is a relatively honest signal because it is released from herbivore-damaged leaves in a variety of species [65], including tomato [56], maize [63], Arabidopsis [67], and poplar $[47,68]$.

Fitness costs incurred by plants exposed to HIPV cues are largely unknown. Generally speaking, induced resistance by plants against herbivores is a cost-savings strategy to restrict the deployment of specialized defensive metabolites until necessary $[5,13]$. Inducible resistance, however, generates a period of vulnerability from the time of attack to the upregulation of resistance [69]. Defense priming via sensory perception of reliable cues may overcome such a vulnerability. However, priming is also an inducible phenomenon and theory therefore predicts that activating defense priming should incur fitness costs that select against maintaining a "primed state" unless HIPVs are detected $[17,70]$. Such fitness costs are predicted to be less severe than would be costs of induced resistance to actual herbivory. Yet, to date, there is limited experimental evidence of such a cost with respect to anti-herbivore priming. For example, wild tobacco (Nicotiana attenuata) exposed to airspace of experimentally clipped sagebrush produce more seeds (i.e., higher presumptive fitness) than did control plants [32]. In contrast, 3-pentanol and 2-butanone field treatments enhanced disease resistance but decrease reproductive output in Cucumis sativa [71], and the non-volatile priming agent $\beta$-amino butyric acid (BABA) reduced seed production in Arabidopsis in a 
dose-dependent manner [72]. Thus, a cost/benefit tradeoff of volatile cue perception in plants is not yet clear for cues associated with insect herbivory.

Volatile application is a promising pesticide alternative may allow for enhanced plant performance in agriculture and for conservation [73]. HIPVs clearly influence interactions among trophic levels and enhance indirect defense $[45,74]$. Moreover, the use of in-field volatile dispensers shows promise as a strategy to enhance plant defenses indirectly by affecting arthropod communities [75]. However, potential fitness costs incurred directly by plants to exogenous volatile cues under field conditions will impact the outcomes of manipulating volatile cues. Current evidence suggests circumstantially that ecological outcomes of exposure to volatile cues may be dependent on environmental variation, but to date there has not been a direct test manipulating multiple plants to a common volatile cue.

Here, I report a common garden field experiment with lima bean (Phaseolus lunatus variety Fordhook 242) and chili pepper (Capsicum annum var. Cayenne) using a single volatile cue designed to test the hypothesis that field plants subject to a persistent dose of a presumably reliable volatile cue incur fitness costs reflected in reduced growth and reproduction. I treated individuals of both species to repeated lowdose applications of $z 3 \mathrm{HAC}$ and measured their growth, reproduction, and herbivore damage throughout the growing season. Based on the theory of defense priming [17], I predicted that exposure to $33 \mathrm{HAC}$-regardless of plant species identity-would reduce growth and reproductive output, while also reducing in-field herbivory. 


\section{Materials and Methods}

\section{$\underline{\text { Study Site and Plants }}$}

A common garden experiment was established on a $54 \mathrm{~m}^{2}$ plot within Blackacre Conservancy's community garden in Louisville, Kentucky (38¹1'33.8"N 85³1'28.3"W;

Appendix 1). The field site was enclosed in a mesh fence to exclude mammalian herbivores. Phaseolus lunatus, Fabaceae, variety Fordham Hook 242 ('lima bean') and the Capsicum annuum, Solanaceae, variety Cayenne pepper, Joe Red Long ('pepper') were chosen as phylogenetically distinct model plants with previously established defense profiles [76], both of which are also agriculturally important. Lima bean is an annual (semelparous) species; pepper is a perennial (iteroparous) species in its native range (USDA, NRCS, 2018). Seeds were purchased from the Louisville Seed Company (Louisville, KY, USA), and germinated in Metromix 510@ in May 2016 in the Biology Department's rooftop greenhouse. After reaching a $20 \mathrm{~cm}$ height, 132 lima bean plants were transplanted to the field May 30, 2016, and 98 pepper plants were transplanted to the field June 28,2016 . While I planned to have an equal sample size, some pepper plants were lost in transport to the field site. Within the field site, plants were planted in alternating rows of twos of lima bean and pepper. Plants were spaced one meter apart from one another in all directions to reduce the influence of neighboring volatile cues and perception of neighboring vials [77].

\section{Volatile exposure}


Plants were acclimated to the field for one week after planting before volatile treatments began. To simulate a naturally occurring low dose $[78,79]$, plants were exposed to lanolin infused paste equivalent to $10 \mathrm{ng} / \mathrm{hr}$ of $z 3 \mathrm{HAC}$. This concentration is $25 \%$ of that which primed poplar [47] and maize [63] previously. A treatment vial contained $50 \mathrm{mg}$ of a $30 \mathrm{ng} / \mu \mathrm{L} \mathrm{z3HAC/lanolin,} \mathrm{while} \mathrm{a} \mathrm{control} \mathrm{vial} \mathrm{contained} 50 \mathrm{mg}$ of lanolin. Each glass vial had a $9 \mathrm{~mm}$ aperture and was maintained at $-80^{\circ} \mathrm{C}$ until use. Each week, both the $33 \mathrm{HAC}$-infused lanolin vials and lanolin-only controls were placed at the bottom of their respective plants. Each vial was inverted and supported with a wire stand and each vial was wrapped in aluminum foil to reduce photo-degradation [75] (Appendix I). Plants were randomly assigned to either z3HAC treatment (lima bean $n=63$; pepper $n=35$ ) or lanolin control (lima bean $n=72$; pepper $n=43$ ) and resulted in uneven survival counts. The unit of replication was an individual plant and each plant received its own vial. Random assignment of treatments was made using blocks of 4 adjacent plants; block was included as a random factor in statistical models, and was not a significant factor in any of the models.

Growth, biomass, and reproduction measurements

I measured height and total leaf counts three to four times during the field season on the experimental plants. Leaves were only counted if they were wider than 2 $\mathrm{cm}$ across for both species while height measurements for both species were recorded from the base of the plant to the uppermost branching point. For lima bean, height was determined by measuring the longest runner within the bush, while pepper plants were measured from the base of the main stalk to the highest branching point. Along with 
height, the total number of leaves per plant was measured three times during the field season. A complete biomass harvest was conducted on pepper for leaves, fruits, roots, and stems at the end of the field season. All leaves and fruits were separated into paper bags before individual plants were extracted from of the ground. After removal, roots and stems were separated, roots were washed with water to remove dirt, and placed into separate paper bags. All materials were dried at $60^{\circ} \mathrm{C}$ for 24 hours and then weighed. A biomass harvest for lima bean was not performed because an Epliachna varivestis (Mexican Bean Beetle) outbreak late in the season removed much of the leaf tissue before I could determine reliable biomass measurements.

As proxies for reproductive fitness, I measured initial flower bud, flower and fruit onset as well as total flower and fruit production. Flowers were recorded if they were true flowers with fully mature pistils and stamen. If a flower was not fully mature, it was recorded as a flower bud. Fruits were recorded as soon as fruit development was observed with either initial pod or exocarp development. Fruit and flower counts per plant were recorded along with the number of mature and immature fruits three times throughout the field season with three to four weeks between measurements.

From the fruits harvested from the final biomass harvest, $\sim 10$ randomly selected, mature fruits from each pepper plant were chosen for seed count analysis from the 188 fruits from z3HAC-treated plants and 210 fruits from controls. Although I observed differences in fruit production between treatments, I randomly selected fruits for analysis to ensure the sample size was the same. Dried fruits were dissected with a scalpel and all seeds were isolated and counted. 


\section{Herbivory}

Since previous work has shown that $z 3 \mathrm{HAC}$ enhances induced defense against herbivores through defense priming $[47,80]$, I monitored herbivory throughout the season. Leaf chewing damage was assessed for both pepper and lima bean as percent leaf area removed (LAR) using a visual estimation technique $[81,82]$ with the following damage categories: $0 \%, 0-5 \%, 5-15 \%, 15-30 \%, 30-50 \%, 50-70 \%, 70-90 \%$, and $>90 \%$. For each damage assessment, every leaf on a plant was categorized into one of the damage categories, and an overall percent damage was determined by averaging the midpoints from each damage category by the total number of leaves within each damage category. Plants were also routinely monitored for the presence of naturally occurring chewing and piercing/sucking herbivores. In particular, I noted a natural occurrence of the black bean aphid (Aphis faba), and recorded its presence/absence on lima bean plants in the field.

\section{$\underline{\text { Statistical analyses }}$}

All statistical analyses were performed in R with the Ime4 and multcomp packages (version 3.4.2)[83]. Growth data, such as plant height, leaf area removed, and flower counts, were analyzed using repeated measures ANOVA with the aov function with a Gaussian distribution.

For repeated measures analyses, such as floral bud investment, I treated date as a within-subjects effect and treatment as a between-subjects effect for all analyses. If the repeated measures ANOVAs were significant, I followed up the analyses with oneway ANOVAs at each time point. For remaining responses, such as biomass data, were 
analyzed using one-way ANOVA (gImer in R). If ANOVA results were significant, I followed these analyses with Tukey's post hoc comparisons.

Results

Treatment with z3HAC differentially affected the growth of lima bean and pepper plants. On average, $z 3 \mathrm{HAC}$-treated lima bean grew $11 \%$ taller compared to control plants throughout the field season (Fig.1a) and produced $17 \%$ more leaves overall than did controls (Fig.1b). In contrast, z3HAC-treated pepper plants were $12 \%$ shorter relative to controls (Fig.1c) and produced 23\% fewer leaves over the field season (Fig.1d). Consistent with height and leaf counts, z3HAC treatment reduced overall biomass of pepper plants by $24 \%$ on average. I destructively harvested all pepper plant biomass at the end of the season. z3HAC-treated pepper plants had lower leaf, stem, and root dry biomass by $21 \%, 31 \%$, and $29 \%$, respectively (Fig.2a-c). Despite these z3HAC-mediated effects on biomass exposure, the aboveground-to-belowground biomass ratio was similar regardless of treatment (Fig. $2 d$ ). That is, pepper plants treated with $z 3 \mathrm{HAC}$ were smaller relative to control plants.

z3HAC treatment also differentially affected reproductive output between the two species, and lowered fruit output in pepper. Flower production was $30 \%$ higher in lima bean plants exposed to z3HAC (Fig.3a), while z3HAC-treated peppers produced 37\% fewer flowers relative to control plants (Fig.3b). z3HAC-treated pepper plants also produced $23 \%$ fewer fruits overall relative to controls (Fig.4a), and the fruits that were 
produced by z3HAC-treated plants had lower wet and dry masses (Fig.4b-c), and 10\% lower total seed counts (Fig.4d) and total seed masses (Fig.4e), relative to controls. Even though total fruit and seed production was reduced by z3HAC treatment, the ratio of seed mass to fruit mass was similar between z3HAC-treated and control plants (Fig.4f). Moreover, the estimated mass of an individual seed was similar between z3HAC-treated plants and controls in pepper (Appendix I). There was no apparent difference in lima bean pod production (Appendix I). However, an unexpected field-wide premature pod drop (independent of treatment) prevented us from determining lima bean seed production with confidence.

z3HAC exposure reduced natural herbivory in lima bean but not pepper plants. Chewing herbivory on pepper plants was low throughout the season and statistically higher in z3HAC-treated plants; however, this effect was driven by only the first assessment date (Fig.5a). In contrast, chewing damage to lima bean leaves increased as the field season progressed, with $z 3 \mathrm{HAC}$-treated plants having overall $26 \%$ less chewing damage than did control plants (Fig.5b). In addition to chewing herbivory, black bean aphids (Aphis faba) colonized 87\% of the z3HAC-treated lima bean plants, compared with only $21 \%$ of control plants (Fig.5c). A. faba colonized early in the season and was only observed June 15-31 (Julian dates 166-181) because a heavy rainfall event reduced their population to undetectable levels. Piercing/sucking herbivores were rare for the remainder of the experiment. 


\section{Discussion}

I show that a persistent, low-dose application of z3HAC differentially affects growth and reproduction in two important agricultural plants in field conditions. Based on previous work on plant defense priming and sensory perception of volatiles $[49,63], \mathrm{I}$ hypothesized z3HAC application would decrease growth and reproductive fitness in both plant species. The rationale for this hypothesis was a central assumption of induced resistance theory that ecological costs modulate the deployment of particular defensive phenotypes until necessary $[15,84]$. Volatile-mediated priming, even if regulated by a different mechanism from resistance [85], is an inducible phenomenon that should incur such fitness costs. Yet, our results clearly indicate that pepper and lima bean had divergent fitness outcomes when subjected to a single GLV under identical field conditions.

Differences in life history traits among plant species may influence the outcome of defense priming. Under significant herbivore stress, short-lived semelparous (annual) species may invest more into reproductive output when exposed to herbivory [86], whereas iteroparous (perennial) species may reserve resources for growth and reproduction for times when herbivores are absent $[87,88]$. Additionally, annuals may optimize reproductive output over seed quality to increase progeny success, where perennials may do the opposite $[89,90]$. It is plausible that herbivore-associated cues such as $z 3 \mathrm{HAC}$ may induce similar divergent fitness effects between annual and perennial species as those induced by herbivory. Previous work on the role of HIPVs in plant anti-herbivore resistance focused on priming-mediated defense with consistent 
results between annual and perennial species: wheat $[80,91]$, corn $[54,78]$, lima bean $[50,53,92]$, tomato [93], blueberry [57], sagebrush [31], and poplar [47] all show evidence of defense priming and enhanced resistance. In contrast, I specifically focused on indicators of plant fitness in a semelparous species (lima bean) and an iteroparous species (pepper) in a common garden array. Consistent with effects observed with direct herbivory, z3HAC treatment alone increased growth and flowering in lima bean, while reducing growth and reproductive output in pepper. Divergent fitness effects from exposure to a single ubiquitous herbivore-associated cue indicate functional similarity in the mechanisms by which annual and perennial plants modulate responses to herbivory as well as volatile indicators of herbivory.

Resource allocation between different tissues is pivotal for growth, reproduction, and defense, and can be influenced by environmental stress. For example, direct herbivory alters resource allocation between aboveground tissue and belowground tissue $[82,94,95]$, as does application of the anti-herbivore phytohormone jasmonic acid [96, 97]. Volatile cues can also affect biomass allocation. For example, barley exposed to volatiles from unwounded neighboring plants of different cultivars increases root and leaf biomass [98], while exposure to volatiles decreases aboveground biomass in other systems $[7,99]$. In our case, volatile treatment reduced overall aboveground and belowground biomass in pepper, but did not appear to alter overall biomass allocation patterns. In other words, z3HAC-treated pepper plants were smaller overall, and therefore produced fewer seeds. 
Flower and fruit production is a key component of plant fitness potential. I showed that $z 3 \mathrm{HAC}$ treatment alone differentially affected flower production in lima bean and pepper. Insect herbivory can increase or decrease floral production depending on the system and environmental conditions [100-102]. Whereas increased flower production is a strategy assumed to ameliorate fitness losses in the presence of an environmental stress $[103,104]$, decreased flower production may be related to costs of chemically mediated defense [105]. Additionally, herbivory affects floral attractiveness $[106,107]$, which may ultimately influence fitness [108]. Our data indicate that a volatile cue alone is sufficient to trigger changes in floral biology, but the magnitude and direction of those changes are plant species-specific. Additionally, z3HAC-treated pepper produced fewer fruits but lima bean did not. The mechanisms underlying $z 3 \mathrm{HAC}-$ mediated effects on flower and fruit production are unknown, but may be similar to those induced by herbivory [100].

Application of an exogenous volatile may impact ecological communities in both expected and pleiotropic ways. HIPVs are well-established mediators of multitrophic antagonistic and mutualistic interactions [109-111], and manipulations of chemical signals and volatile blends have been used for biological control in a wide range of systems [73, 112]. For example, HIPV-infused sticky traps in a grape (Vitis vinifera) orchard differentially attracted lacewings, hoverflies, and parasitoids [113]. Exogenous GLV manipulation using "dispensers" under field conditions altered the arthropod community composition in maize [75]. In our study, A. faba were clearly and unexpectedly attracted to z3HAC-exposed plants (Fig. 5). Under glasshouse conditions, 
A.faba were repelled by $z 3 \mathrm{HAC}$ alone [114], which suggests that the cue that mediated attraction was not our treatment alone. It is tempting to speculate that aphid attraction combined with reduced chewing herbivory in lima bean may be reflective of $z 3 \mathrm{HAC}$ effects on Jasmonic Acid (JA) and Salicylic Acid (SA) signaling, which would be consistent with a JA-SA tradeoff $[4,115]$. Ultimately, however, the utility of GLVs in field applications will depend on understanding community-level effects of the application.

Volatile identity, concentration, and duration may affect the reliability of a cue. Plants experiencing insect herbivory frequently generate species-specific blends of volatile compounds $[29,116]$, which can influence fitness in neighboring plants $[40,117$, 118]. Plant-derived compounds associated with herbivory include GLVs [47, 54, 99], phenylpropanoid derivatives [55], and terpenes [119]. However, individual compounds within a blend can affect plant defense and priming as much as the blend. GLVs are particularly reliable herbivore-associated cues because they are shared among a variety of species [29] and are released by wounded plant material [65]. z3HAC was used in this study because it is released primarily from herbivore-damaged leaves [63] and has a demonstrated priming ability [47]. Additionally, concentration of a cue influences resistance [99]. For example, a repeated, low-dose exposure to a GLV enhances plant resistance compared to a single application [79]. For these reasons, I chose to use a relatively persistent, low-dose exposure to $z 3 \mathrm{HAC}$ of $10 \mathrm{ng} / \mathrm{hr}$ ( $25 \%$ of the concentration that primed poplar [47] and maize [63]), that resulted in differential fitness effects between lima and pepper. 
In summary, our key finding is that persistent application of a low dose of a single volatile compound $z 3 \mathrm{HAC}$, a common HIPV and GLV, in field conditions leads to divergent growth and reproductive fitness effects between two plant species with different life histories. HIPVs have the potential to impact agricultural and conservation practices. However, the utility of plant-derived HIPVs in enhancing plant defense will depend on understanding how plant life history, physiology, and other ecological factors influence whether a target plant will benefit from the HIPVs or not. 

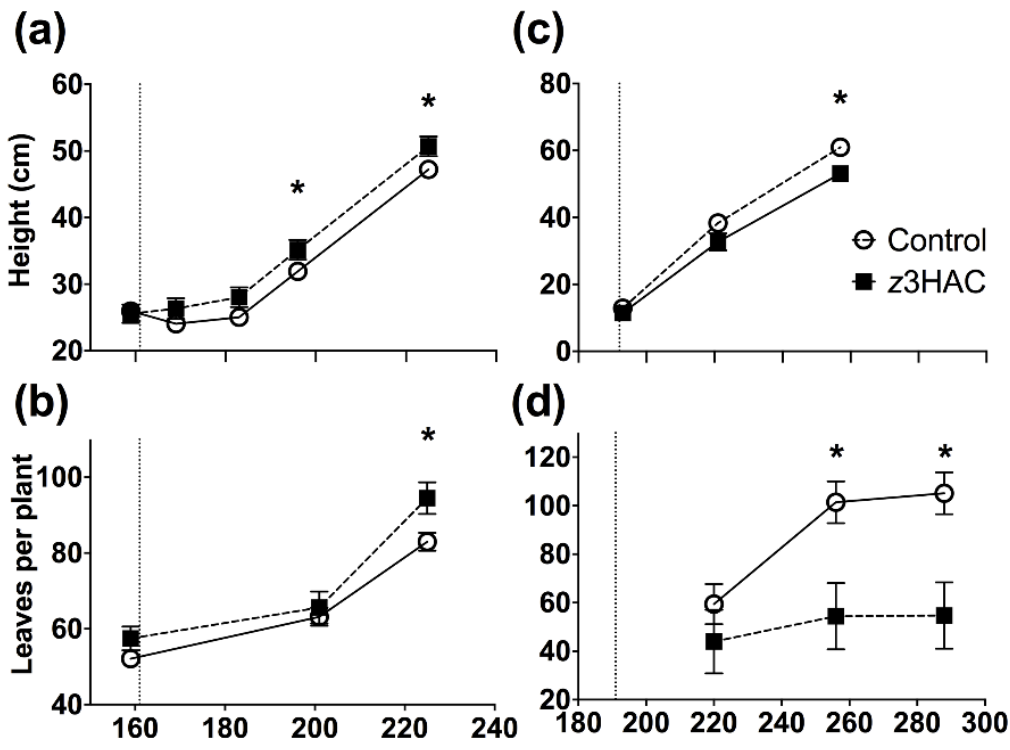

Date (Julian Date) 2016

Figure 1. Height measurements and leaf counts for Phaseolus lunatus (lima bean) and Capsicum annuum (pepper) grown in a common garden field experiment. Height for

(a) lima bean and pepper (c). Leaf counts for (b) lima bean and (d) pepper included all mature leaves on each plant. Open circles represent control plants (receiving lanolinfilled vials); filled squares represent persistent application of $10 \mathrm{ng} / \mathrm{hr}$ cis-3-hexenyl acetate (z3HAC) dissolved in lanolin. Dropdown lines indicate the initial application of z3HAC treatment: lima bean and pepper plants were first exposed on June 10, 2016 (Julian date 161) and July 11, 2016 (Julian date 192), respectively. Points represent averages +/- SE. Repeated measures ANOVAs (aov in R) were followed by post-hoc oneway ANOVAs at each time-point. Asterisks $\left({ }^{*}\right)$ represent $P<0.05$ between treatment and control at a given time point. 
(a)

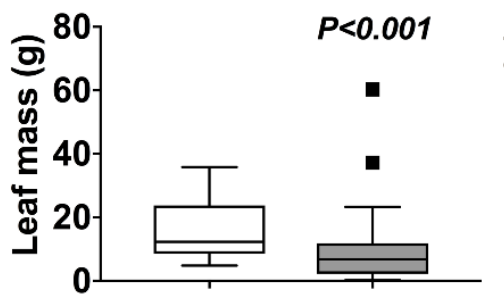

(b)

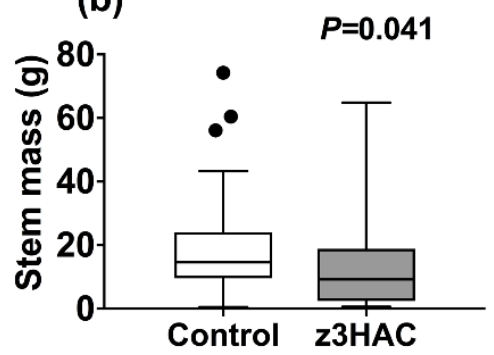

(c)

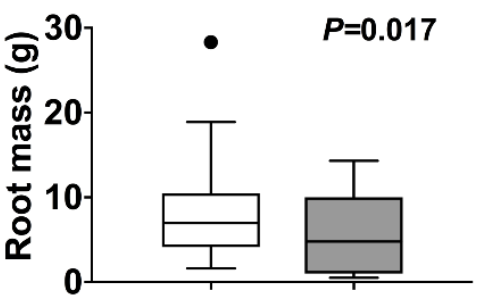

(d)

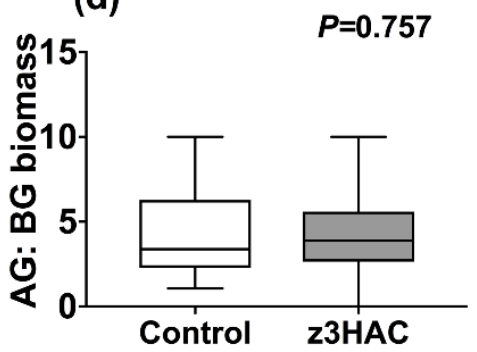

Figure 2: Biomass measurements of field-grown Capsicum annuum (pepper) plants. (a)

Leaf, (b) stem, (c) root biomass, and (d) the aboveground:belowground biomass ratio in C.annuum plants were determined at the end of the field season following destructive harvest. Box plots represent the raw data ranging from the upper to the lower quartiles and the median. Error bars represent the 5\% and 95\% Confidence intervals of the data, and individual dots are observations that fell outside of those parameters. $P$ values represent Tukey's HSD comparisons. 
(a)

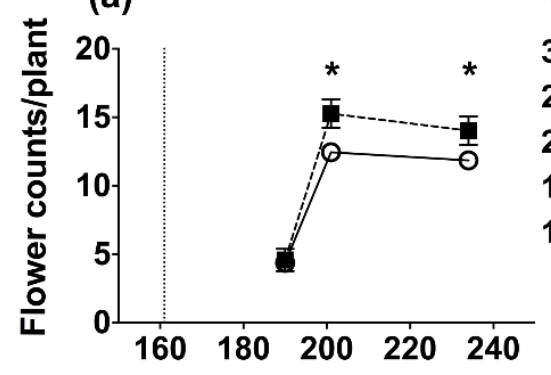

(b)

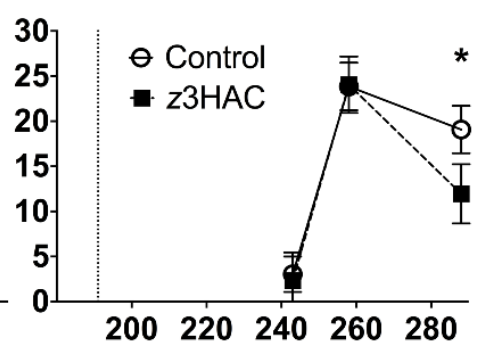

Date (Julian) 2016

Figure 3: Total flower production in (a) Phaseolus lunatus (lima bean) and (b) Capsicum annuum (pepper). Open circles represent control plants (receiving lanolin-only vials); filled squares represent plants receiving a persistent application of vials containing 10 $\mathrm{ng} / \mathrm{hr}$ cis-3-hexenyl acetate $(\mathrm{z} 3 \mathrm{HAC})$ dissolved in lanolin. Dropdown lines indicate the initial application of $z 3 \mathrm{HAC}$ treatment: lima bean and pepper plants were first exposed on June 10, 2016 (Julian date 161) and July 11, 2016 (Julian date 192), respectively. Points represent averages +/- SE. Repeated measures ANOVAs (aov in R) were followed by one-way ANOVAs at each time-point. Asterisks $(*)$ represent $P<0.05$ between treatment and control at each time point. 

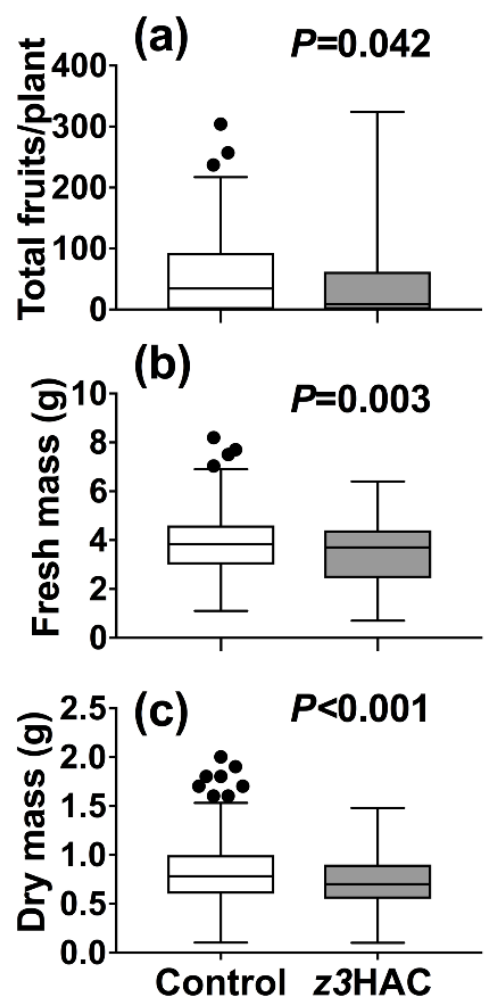

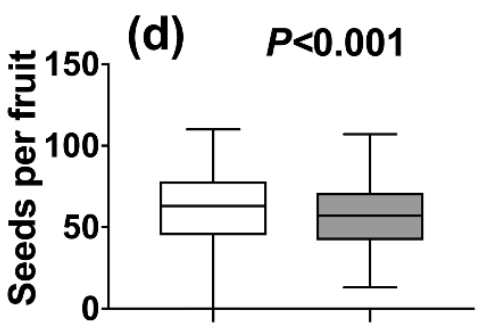

(e)
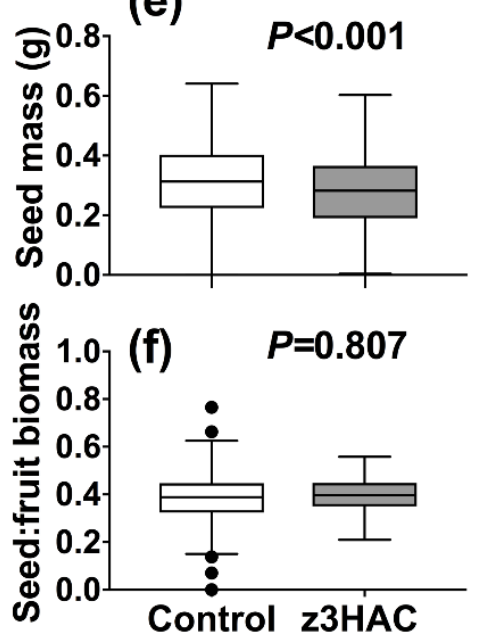

Figure 4: Fruit and seed production in Capsicum annuum (pepper) plants grown in a common garden experiment treated with a persistent application of the green leaf volatile cis-3-hexenyl acetate (z3HAC). The (a) total number of fruits were counted in the field, and (b) wet and (c) dry masses fruit masses were determined in the lab. (d) The total number of seeds per fruit and (e) the estimated mass per seed were determined from a subset of the fruits produced. (f) The ratio of seed mass to fruit mass was calculated to assess the efficiency of seed production. Box plots represent the raw data ranging from the upper to the lower quartiles and the median. White boxes represent control plants; gray boxes represent plants treated with z3HAC. Error bars represent the $5 \%$ and $95 \%$ confidence intervals and individual dots are observations that fell outside of those parameters. P-values represent Tukey's HSD comparisons. 

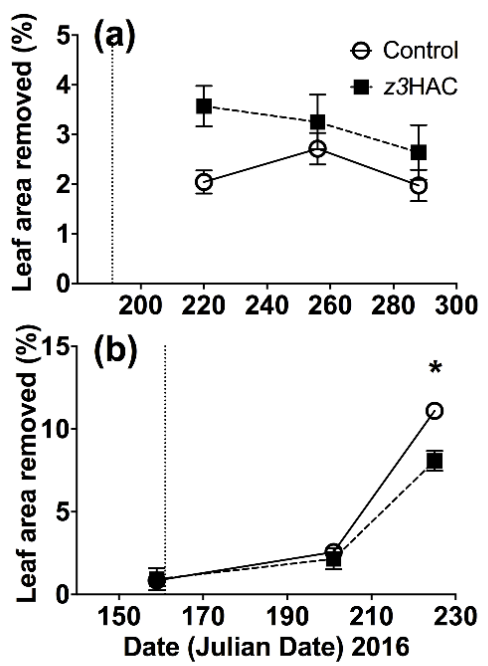

(c)

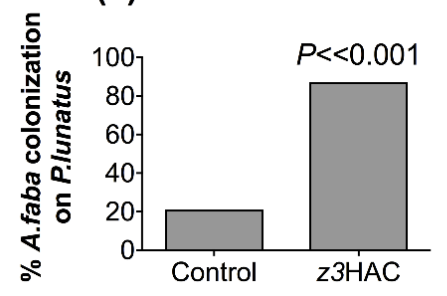

Figure 5: Herbivore damage on Capsicum annuum (pepper) and Phaseolus lunatus (lima bean) plants in a common garden field experiment. Chewing damage on (a) pepper and (b) lima bean plants were quantified overtime (see methods). Open circles represent control plants (receiving lanolin-filled vials); filled squares represent persistent application 10ng/hr cis-3-hexenyl acetate (z3HAC) dissolved in lanolin. Dropdown lines indicate the initial application of $z 3 \mathrm{HAC}$ treatment. Points represent averages +/- SE. Repeated measures ANOVAs (aov in R) were followed by one-way ANOVAs at each time-point. Asterisks $\left({ }^{*}\right)$ represent $P<0.05$ between treatment and control at each time point. (c) Aphis faba colonization on lima bean plants. Bars represent the percentage of plants in each group where $A$.faba were observed $\left(\chi^{2}=\right.$ 50.11, $\mathrm{df}=1, P<0.001)$. 


\title{
CHAPTER III \\ GROWTH-DEFENSE TRADEOFFS OF EAVESDROPPING ARE AFFECTED BY DURATION OF EXPOSURE TO A GREEN-LEAF VOLATILE
}

\author{
Summary
}

Green-Leaf Volatiles (GLVs) are a class of herbivore-induced plant volatiles that are ubiquitously-released chemical signals in plants following herbivory. These signals are reliable cues for neighboring plant defense priming against herbivory, but are often associated with fitness costs for eavesdropping plants. Since GLV duration corresponds to timing and severity of herbivory, eavesdropping costs should be affected by GLV duration. Specifically, continuous GLV exposure is expected to result in increased investment towards defense and away from growth, while short-term GLV exposure should have little effect on growth while still increasing defense. I tested these predictions in a field experiment, exposing Phaseolus lunatus to one of five volatile treatments involving the GLV cis-3-hexenyl acetate (z3HAC): either continuous exposure for 120 days, a single 1 week pulse (Transient) starting at day 30,60, or 90 of the experiment, or a weekly 24 hour exposure (Pulsed). I found that continuous and pulsed exposure to $z 3 \mathrm{HAC}$ increased biomass, while transient treatments had no effect on plant biomass or reproductive output. Interestingly, I found no effect of z3HAC exposure on in-field defense metrics, such as quantifiable cyanide and leaf-area removed. While I 
initially predicted a direct correlation between growth and defense, growth-defense tradeoffs may be more nuanced than initially predicted. 


\section{Introduction}

Green-Leaf Volatiles (GLVs) are C6 fatty acid derivatives that are ubiquitously released in plants following herbivory and are a class of herbivore-induced plant volatiles [24]. Numerous studies have demonstrated that GLV exposure generates molecular $[47,54,120]$ and chemical changes $[121]$ in plants that result in enhanced resistance profiles relative to unexposed plants [16, 40, 50, 122]. However, such inducible defenses are often associated with fitness costs $[17,69,123]$. While extensive work has been conducted to assess costs of induced defense, little work has been conducted to date to assess costs related to GLV-mediated eavesdropping.

Since GLV emissions reflect stress experienced by an emitting plant [124], the benefits and costs of eavesdropping may vary with duration of GLV exposure. Herbivores in natural systems range in seasonality, density, frequency and feeding intensity, consequently impacting GLV emissions and potential fitness effects for eavesdroppers. This is especially true in agricultural crops such as lima bean, where different herbivores are present throughout the field season. For example, the Mexican bean beetle is a voracious pest that undergoes multiple, asynchronous generation cycles within a field season [125], resulting in a continuous release of GLVs from damaged plants. Conversely, lepidopterans such as Spodoptera exigua and Spilosoma virginica may have only 2-3 generations per season, with caterpillars feeding gregariously on leaves for only one to two weeks over thirty days [126-128], generating periodic but intense GLV bursts. Still other herbivores, such as Aphis faba, feed temporarily early on 
in the season on lima bean before host switching [129]. Therefore, perceived risk in an eavesdropping plant affects costs and can be related to duration of exposure to a cue. For example, eavesdropping on transient herbivore oviposition cues resulted in increased reproductive output in eavesdropping Brassica sp. [130] while exposure to cues due to continuous herbivory by specialists resulted in altered rhizome growth in goldenrod [131]. Since differences in herbivore feeding frequency, and consequent volatile duration, can indicate severity of herbivory, volatile duration may have significant implications for the costs of eavesdropping $[9,70]$.

In this study, I examined how timing and duration of plant exposure to the GLV cis-3-hexenyl acetate (z3HAC) affected growth, reproduction, chemical defenses, and herbivory in Phaseolus lunatus (Lima bean). I expected that continuous z3HAC exposure would incur greater net fitness benefits by increasing induced defenses and defensive priming responses (ability of plants to quickly respond to herbivory) thereby reducing herbivory at the cost of reduced vegetative growth. Additionally, I expected that short periods of exposure to GLVs would incur little to no fitness affects [123] while conferring enhanced defense profiles via cyanogenic potential.

Materials and methods

Experimental Design

To determine if GLV exposure alone would influence long term fitness and defense priming in plants, I exposed $P$. lunatus plants to five different GLV exposure regimens that differed in timing and duration (Fig. 6) within a common garden 
experiment during the 2017 growing season (May-Aug). In May 2017, 435 plants were started in the greenhouse at the University of Louisville and when $14 \mathrm{~d}$ old, were transplanted in a $950 \mathrm{~m}^{2}$ area within the community garden at Blackacre Conservancy in Louisville, KY, USA (38.2N, -85.7W). Plants were placed $1 \mathrm{~m}$ apart in all directions to reduce interplant communication $[50,77]$. Each plant was randomly assigned to one of five specific GLV treatments, described below. Additionally, plants were assigned biomass harvest dates at either 40, 70, or $100 \mathrm{~d}$ post-treatment.

I selected the GLV compound cis-3-hexenyl acetate (z3HAC) for this experiment as it is released during herbivory in P. lunatus, making it a reliable, honest signal. For example, spider mite damage increased $z 3 \mathrm{HAC}$ emissions by $100 \%$ relative to controls under greenhouse conditions [132] while simulated chewing damage and phytohormone application also increased z3HAC emissions $[59,133]$. z3HAC was administered using amber vials filled with a lanolin paste $[75,134]$. Additionally, all vials were weighed, numbered, and tracked throughout the field season to ensure consistent volatile administration similarly to chapter 2 . For the continuous exposure treatment, z3HAC-infused lanolin vials were replaced weekly throughout the experiment to maintain a consistent $\sim 10 \mathrm{ng} \mathrm{h}^{-1}$ emission rate. Pulsed exposure plants were exposed to a $24 \mathrm{~h}$ dose of z3HAC on a weekly basis followed by $6 \mathrm{~d}$ of control (lanolin without $z 3 \mathrm{HAC}$ ) vials until they were harvested, while transient exposure plants involved $z 3 \mathrm{HAC}$ exposure for one week, with control vials during the rest of the season. Three separate weeklong transient $z 3 \mathrm{HAC}$ exposures at 30,60 , or $90 \mathrm{~d}$ after initial introduction were applied within the field site. Control treatments involved vials with pure lanolin were 
placed at the base of each plant throughout the field season (Fig. 6). During our initial analyses, I found that timing of the transient treatment had no effect on our observed results. Therefore, data from all transient treatment plants, regardless of exposure timing, were combined for further analyses and presented below.

\section{$\underline{\text { Response measurements }}$}

Biomass: As an approximate measure for overall growth, I scheduled destructive biomass harvest dates throughout the field season. Plants were randomly assigned a destructive harvest date at 40,70 , or $100 \mathrm{~d}$ after experiment initiation, which corresponded to biomass harvest dates occurring $10 \mathrm{~d}$ after each transient exposure to control for time of collection across our treatments and to account for transient effects on metabolite profiles in leaf collections [79]. Harvested plants were uprooted and placed into large brown paper bags and returned to the lab for separation by tissue type. Plants were separated into leaf, stem, fruit, and root tissue. Root tissue was hand washed in water to remove all dirt before drying and all plant materials were dried at $60^{\circ} \mathrm{C}$ for $24 \mathrm{~h}$ before weighing.

Reproduction: Floral bud and flower counts were initiated when they were first observed in the field. For floral buds, these counts occurred on days 4, 25, and 69, while flowers were counted on days 5, 42, and 80. Fruit counts were conducted three times on days 30,60 , and 90 . For each census, 100 plants were randomly selected and all flower buds $>0.2 \mathrm{~cm}$ in length, all mature flowers (with pollen present), and all pods $>$ $1 \mathrm{~cm}$ in length were counted. 
Defense priming response: Defense priming is defined as an organism's ability to respond more quickly and aggressively upon exposure to a stress stimulus after previous exposure to a signal or cue (aka eavesdropping) [16]. To quantify priming in our experiment, I expected that previous exposure to $z 3 \mathrm{HAC}$ would result in increased defense induction when directly damaged by an herbivore compared to a plant that was directly damaged by an herbivore without previous z3HAC exposure. I generated an induced defense response $[16,17,123,135]$ by applying Spodoptera exigua oral secretion (OS) + mechanical damage. Spodoptera exigua is a common generalist herbivore and its oral secretions are often used to initiate defense induction [136] and it is a known herbivore on lima bean [137]. I tagged one photosynthetically mature leaf per plant [78] in July from 150 plants. While these leaves were still on the plant, I wounded these leaves by crushing $20 \%$ of the leaf area with pliers and then applying 10uL of a 1:4 S.exigua OS : water solution to damaged areas $[138,139]$. I collected damaged leaves $24 \mathrm{~h}$ later along with nearby undamaged leaves on the same plant for controls. Then leaves were flash frozen in liquid nitrogen and stored at $-80^{\circ} \mathrm{C}$ prior to powdering and lyophilization.

After lyophilization, I analyzed cyanogenic potential to assess defense priming. Since cyanogenic potential is a putative defense mechanism in Lima bean [140, 141], I expected to see differences in consequent induction profiles. In our case, if $z 3 \mathrm{HAC}$ acted as a priming agent, then I would expect to see increased cyanogenic potential in z3HAC plants that were consequently damaged compared to solely wounded leaves. A smallscale cyanide colorometric quantification was modified from Gleadow et al. (2011) for 
rapid quantification of total cyanide [134, 142]. Briefly, $5 \mathrm{mg}$ of lyophilized tissue was weighed and placed into $2.0 \mathrm{~mL}$ centrifuge tubes and placed on ice. Once all the samples were on ice, $200 \mu \mathrm{L}$ of cold citrate buffer (0.1 M, pH 5.5-6.5) and a $200 \mu \mathrm{L}$ vial glass insert (Agilent part number 5183-2090) with 1.0M NaOH was placed inside the $2 \mathrm{~mL}$ tube before capping. After a 15 -hour incubation at $37^{\circ} \mathrm{C}, 30 \mu \mathrm{L}$ of the $0.1 \mathrm{M}$ solution was added in triplicate in a 96 well plate followed by $30 \mu \mathrm{L} 0.5 \mathrm{M}$ acetic acid, $75 \mu \mathrm{L}$ of reagent a $\left(5.0 \times 10^{-3} \mathrm{~g} \mathrm{ml}^{-1}\right.$ succinimide, $5.0 \times 10^{-4} \mathrm{~g} \mathrm{ml}^{-1} \mathrm{n}$-chlorosuccinimide $)$ and $30 \mu \mathrm{L}$ of reagent $b\left(0.03 \mathrm{~g} \mathrm{~mL}^{-1}\right.$ barbituric acid, $30 \%$ pyridine deionized water). Concentration was calculated by comparing absorbance values at $580 \mathrm{~nm}$ to our standard curve ranging from 0-3000 $\mu \mathrm{mol}$ of cyanide.

Herbivore damage: Along with cyanogenic potential, I monitored in-field resistance to herbivory as a defense metric. This metric was included to account for realtime herbivore preference and performance. To assess in-field herbivory, I monitored leaf area lost (LAR) at 30,60 , or $90 \mathrm{~d}$ on a rotating subset of plants in the experiment. During each collection date, I collected data from 20 randomly selected plants per treatment. I randomly selected 50 leaves per plant and classified each leaf into the following damage categories: $0 \%, 0-5 \%, 5-15 \%, 15-30 \%, 30-50 \%, 50-70 \%, 70-90 \%$, and $>90 \%$ damage. I then calculated the average damage level from the midpoint of each category for a given leaf to give one LAR value per plant [143].

\section{$\underline{\text { Statistical analyses }}$}

Analyses were conducted in R studio version 1.2.1335 with base $\mathrm{r}$ version Arbor Day [83] using the Ime4, multcomp, and tidyr packages [144, 145]. I used one-factor 
ANOVAs followed by Tukey's posthoc tests at each sampling date to compare differences in reproduction, defense, biomass, and herbivory across z3HAC exposure treatments. I could not conduct repeated measures ANOVAs on these data sets since a subset of plants was used on different sampling dates. All response variables were checked for normality and log-transformed as necessary to meet model assumptions.

Results

Growth and Reproduction: Overall biomass was the highest 70 days after the start of the experiment with continuous and pulsed exposure to $z 3 \mathrm{HAC}$ resulting in the greatest investment in overall biomass, while transient exposure had no overall effect on total biomass (Fig. 7, Table 1). Interestingly, different parts of the plant were influenced by z3HAC exposure treatments (Fig. 7, Table 1). While leaf and root biomass were not affected z3HAC exposure (Fig. 7a and 7c), continuous and pulsed exposure treatments resulted in increased stem biomass at both mid- and late-season dates (Fig. 7b). Although, z3HAC exposure increased biomass, z3HAC exposure had no effect on infield reproductive output (Fig. 8, Table 1).

Defenses: Cyanogenic potential was not significantly impacted by z3HAC exposure treatments (Fig. 9a, Table 1). Additionally, I found no differences in in-field herbivory due to z3HAC exposure (Fig. 9b, Table 1), though in-field herbivore damage increased throughout the field season, with 30-40\% LAR towards the middle and end of the field season. 


\section{Discussion}

This is one of the first studies to show that timing and duration of plant exposure to a single GLV can affect growth-defense tradeoffs under field conditions, but not necessarily in the ways I predicted. I showed that transient z3HAC exposure does not have significant effects on overall growth, while continuous and pulsed exposure significantly increases, rather than decreases, vegetative growth in P.lunatus.

Additionally, pulsed z3HAC exposure increased stem biomass along with continuous exposure to $z 3 \mathrm{HAC}$. However, reproduction ultimately was not affected by any exposure to $z 3 \mathrm{HAC}$. These results are surprising based on our previous work with $z 3 \mathrm{HAC}$ in chapter 2 [134]. Specifically, I predicted that short z3HAC duration would have negligible effects on growth, while increased $z 3 \mathrm{HAC}$ exposure would incur significant growth costs under field conditions, as shown in other annual crops like corn [146, 147]. However, this is not universal. For example, seeds of Medicago trunculata exposed to z3HAC showed increased growth, similar to our results in chapter 2 [148]. Our findings may be due to the fact that lima bean exhibits compensatory growth in response to herbivory [149] and alterations in growth patterns are species-specific.

What is notable about this work is that I observed compensatory growth in eavesdropping plants. Overcompensation can occur at different life history stages [150, 151], and annual plants like lima bean have evolved to invest more heavily in overall growth compared to defense $[87,152,153]$. Overcompensation as a tolerance mechanism is widespread in response to herbivory [154] and within environments with high herbivore pressure, compensatory growth may be a more adaptive response 
relative to investment in costly chemical defenses. Previous work has shown that extensive herbivore damage can increase flowering, seed production, and overall growth in a wide variety of plants $[86,103,150,155]$. To my knowledge, evidence for compensatory vegetative growth after exposure to volatile cues has only been documented in this study in lima bean and in one study on sagebrush [156].

Conversely, GLV exposure had no effect on plant defense priming, at least when measured as cyanogenic potential. While increased overall growth may reflect the response to "escape" under direct herbivore pressure [157], I predicted that it may be worthwhile to invest in defense rather than compensatory growth under shorter $z 3 \mathrm{HAC}$ duration treatments compared to a continuous exposure. Therefore, the lack of differences in cyanogenic potential that I observed may be related to the cost of cyanide production itself and how cyanogenesis is prioritized within a plant. In lima bean, cyanogenesis is an energetically expensive defense response $[158,159]$ that is often restricted to essential organs, such as young leaves and flowers, to reduce costs [76, 160]. When I collected leaves for analysis in July, in-field herbivore damage was beginning to reach severe levels (between 20-25\%), mostly likely due to the increased presences of the lima bean specialist Epilachna varivestis which were the dominant herbivores in our field site (personal observations). As a result of this infestation, I preferentially collected photosynthetically mature leaves since they were readily available and not decimated by E.varivestis larva [76]. In the previous chapter, differences in cyanogenic potential were only found in young "sink" leaves while photosynthetically mature leaves were unaffected by z3HAC (Appendix 1). As a result of 
available leaf materials, I was unable to observe tradeoffs in our leaf samples and reflect within-plant prioritization. Additionally, cyanide-related defenses are efficient deterrents against generalist herbivores while they are less effective at deterring specialists such as Epilachna varivestis [159]. Since plant populations can drastically modify the presence of defense in response to changing herbivore pressure within and across generations $[15,161,162]$, it may have been more adaptive in this case to invest more strongly in growth rather than defense in mature leaves since herbivore pressure was so strong.

While I expected to find a direct effect of GLV duration on growth-defense tradeoffs, I instead found evidence for compensatory growth in response to exposure to z3HAC. This may have occurred because compensatory growth in itself is a tradeoff in the constant presence of herbivores [70]. While short-term GLV exposure had relatively little effects on growth and reproduction, continuous GLV exposure enhanced growth with no obvious fitness tradeoffs. These results indicate that growth-defense tradeoffs may be more nuanced than often predicted, and defense priming itself may be independent of fitness costs. The pulsed and transient treatments reflected more common types of herbivore pressure, while our continuous treatment represented an ecological extreme. Therefore, future studies should investigate other factors related to eavesdropping, such as herbivore identity on an emitting plant and synergistic effects of multiple cues on the costs of eavesdropping. 
Table 1: Summary statistics for growth and defense metrics for P.Iunatus. One-way ANOVAs were conducted at each time point for biomass, reproduction, and defense measures. Significant differences $(P<0.05)$ are in bold.

\begin{tabular}{|c|c|c|c|c|c|c|c|c|c|c|c|c|}
\hline & \multicolumn{3}{|c|}{ Leaf Biomass } & \multicolumn{3}{|c|}{ Stem Biomass } & \multicolumn{3}{|c|}{ Root Biomass } & \multicolumn{3}{|c|}{ Total Biomass } \\
\hline & $\mathrm{df}$ & $\mathrm{F}$ & $\mathrm{p}$ & df & $\mathrm{F}$ & $\mathrm{p}$ & df & $\mathrm{F}$ & $p$ & df & $F$ & $\mathrm{p}$ \\
\hline \multicolumn{13}{|l|}{ Date 1} \\
\hline $\begin{array}{c}z 3 \mathrm{HAC} \\
\text { treatment }\end{array}$ & 3,70 & 0.2096 & 0.8894 & 3,68 & 2.3004 & 0.08503 & 3,71 & 0.4361 & 0.7278 & 3,80 & 0.1768 & 0.9118 \\
\hline \multicolumn{13}{|l|}{ Date 2} \\
\hline $\begin{array}{c}z 3 \mathrm{HAC} \\
\text { treatment }\end{array}$ & 3,69 & 1.6735 & 0.1807 & 3,59 & 2.8577 & 0.04456 & 3,55 & 0.2975 & 0.8271 & 3,34 & 3.2189 & 0.03478 \\
\hline \multicolumn{13}{|l|}{ Date 3} \\
\hline \multirow[t]{3}{*}{$\begin{array}{c}z 3 \mathrm{HAC} \\
\text { treatment }\end{array}$} & 3,155 & 1.4119 & 0.2414 & 3,153 & 2.912 & 0.03635 & 3,131 & 2.1422 & 0.09799 & 3,146 & 2.1933 & 0.09134 \\
\hline & \multicolumn{3}{|c|}{ Bud count } & \multicolumn{3}{|c|}{ Flower count } & \multicolumn{3}{|c|}{ Pod count } & \multicolumn{3}{|c|}{ LAR 1} \\
\hline & $\mathrm{df}$ & $\mathrm{F}$ & $p$ & df & $\mathrm{F}$ & $p$ & df & $\mathrm{F}$ & $p$ & df & $\mathrm{F}$ & $\mathrm{p}$ \\
\hline \multicolumn{13}{|l|}{ Date 1} \\
\hline $\begin{array}{c}23 \mathrm{HAC} \\
\text { treatment }\end{array}$ & 3,405 & 0.8458 & 0.4694 & 3,333 & 0.7421 & 0.5276 & 3,392 & 2.222 & 0.08511 & 3,400 & 0.1302 & 0.9421 \\
\hline \multicolumn{13}{|l|}{ Date 2} \\
\hline $\begin{array}{c}z 3 \mathrm{HAC} \\
\text { treatment }\end{array}$ & 3,392 & 1.1021 & 0.3481 & 3,90 & 1.1954 & 0.3161 & 3,74 & 0.2712 & 0.846 & 3,94 & 0.452 & 0.7165 \\
\hline \multicolumn{13}{|l|}{ Date 3} \\
\hline \multirow[t]{3}{*}{$\begin{array}{c}z 3 \mathrm{HAC} \\
\text { treatment }\end{array}$} & 3,76 & 1.6457 & 0.1859 & 3,70 & 0.1316 & 0.9409 & 3,53 & 0.8767 & 0.4591 & 3,66 & 0.5273 & 0.6651 \\
\hline & \multicolumn{3}{|c|}{ Cyanide } & & & & & & & & & \\
\hline & df & $\mathrm{F}$ & $\mathrm{p}$ & & & & & & & & & \\
\hline \multicolumn{13}{|l|}{ Date 1} \\
\hline $\begin{array}{c}z 3 \mathrm{HAC} \\
\text { treatment }\end{array}$ & 7,108 & 0.7101 & 0.6635 & & & & & & & & & \\
\hline
\end{tabular}




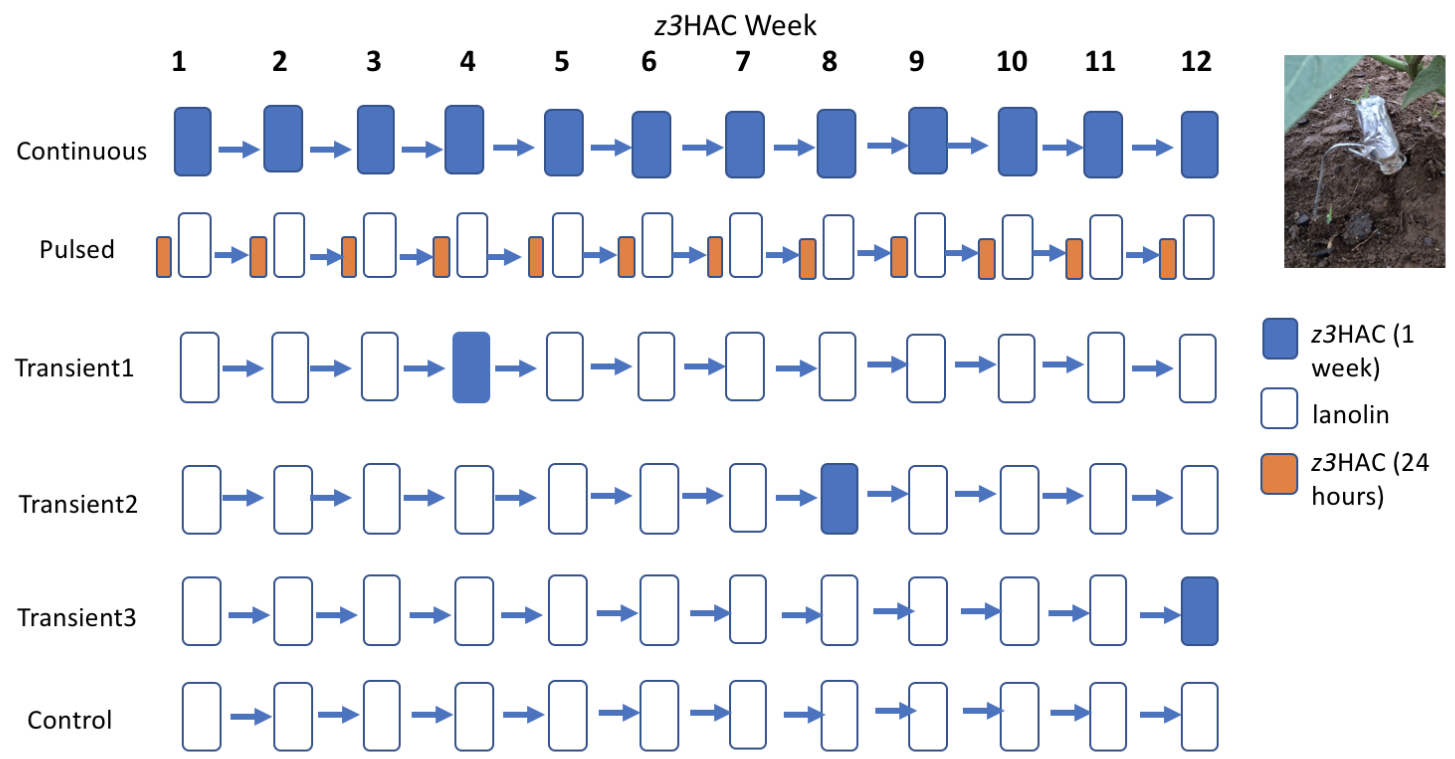

Figure 6. Volatile administration setup. Each rectangle represents a week-long exposure to $1680 \mathrm{ng}$ z3HAC (blue), a 24-hour exposure (orange) or exposure to a control lanolin only vial (white). Continuous exposure plants were given a vial every week throughout the summer while pulsed treatment plants were given a 24-hour dose at the beginning of each week. Transient exposure plants were given a week long z3HAC dose at 4,8 , or 12 weeks after the start of the experiment. 


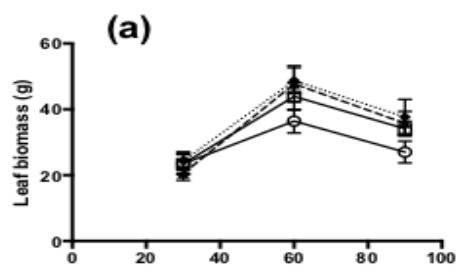

(b)
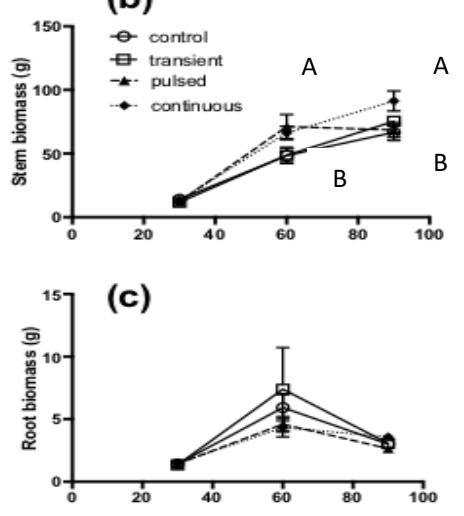

(d)

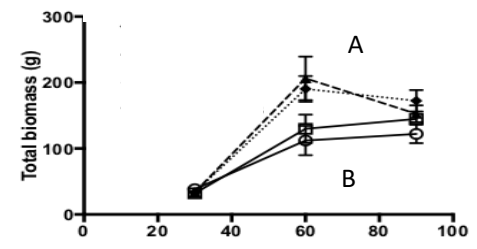

Days post sowing

Figure 7. Volatile duration differentially affects Leaf (a), stem (b), root (c), and total (d) biomass in P.lunatus. One-way ANOVAs were completed at each time point and differences between treatments are denoted with letters. Open circles represent plants exposed to lanolin controls, open squares represent transient exposure plants, closed circles represent pulsed treatment plants, and closed triangles represent continuous exposure plants. Each point represents averages +/- SE. See table 1 for summary statistics. 

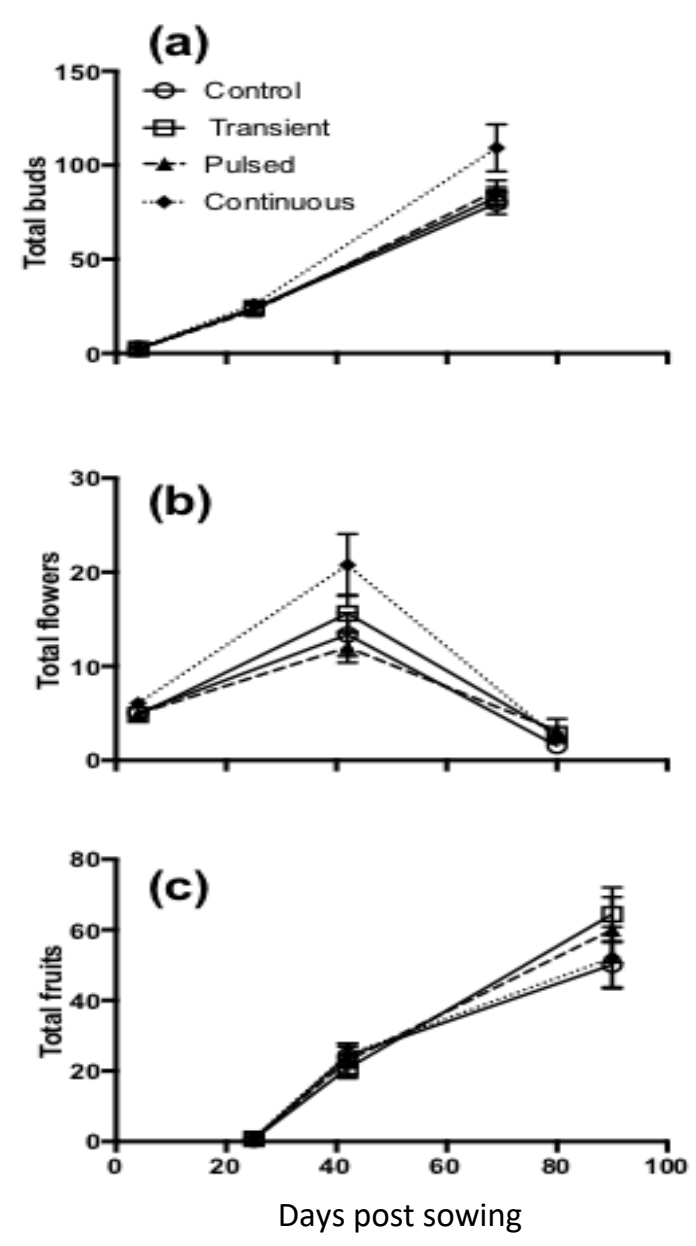

Figure 8. Volatile exposure type does not affect reproductive structures and output in

P.lunatus. Bud (a), flower (b), and fruit (c) counts were measured as outlined in Methods. Z3HAC was introduced on June 1 for P.lunatus. One-way ANOVAs were completed at each time point. Open circles represent plants exposed to lanolin controls, open squares represent transient exposure plants, closed circles represent pulsed treatment plants, and closed triangles represent continuous exposure plants. Each point represents averages +/- SE. See table 1 for summary statistics. 


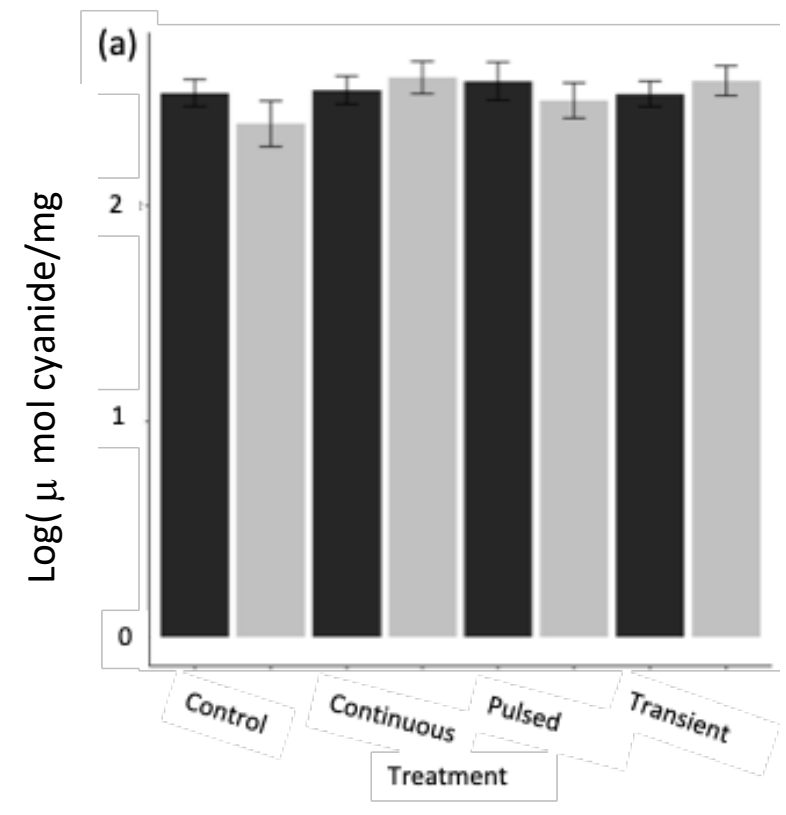

(b)

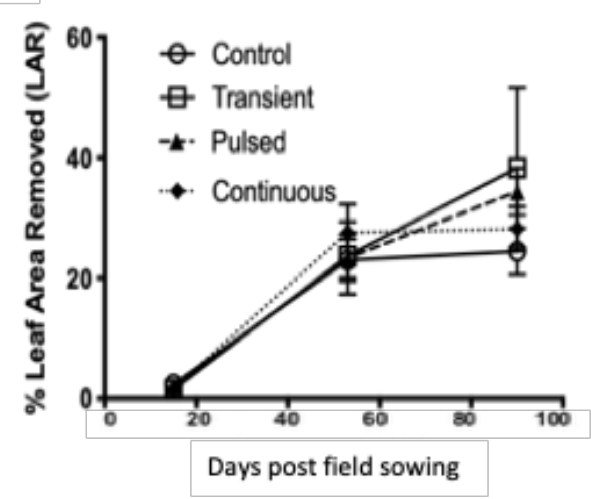

Figure 9. z3HAC exposure duration does not impact cyanide induction (a) or chewing herbivore resistance in the field (b). In part a, designated plants were either left as undamaged controls (black bars) or induced with a mechanical wounding treatment with 1:4 S.exigua oral secretion treatment (grey bars). One-way ANOVAs were conducted at each time point. See table 1 for summary statistics. 


\section{CHAPTER IV \\ HERBIVORE IDENTITY HAS INDIRECT FITNESS EFFECTS VIA HERBIVORE-INDUCED PLANT VOLATILES IN P.LUNATUS UNDER FIELD CONDITIONS}

\section{Summary}

Herbivore-induced plant volatiles (HIPVs) are reliable cues for eavesdropping because they accurately predict herbivore identity and the degree of damage experienced by a plant. Eavesdroppers prepare defense mechanisms in anticipation of herbivory, resulting in more defenses upon herbivory via Defense Priming (DP). While recent studies have shown that HIPV exposure can alter growth patterns in eavesdropping plants, little is known about interacting factors that may influence the costs of eavesdropping. To determine if costs of eavesdropping vary by herbivore identity and emitting plant identity, I experimentally manipulated emitter and eavesdropper plant identity using two cultivars of lima bean, Henderson and Fordhook, within a common garden experiment. Emitter plants were either left undamaged, damaged by the generalist herbivore Spodoptera exigua, or damaged by the legume specialist herbivore Anticarsia gemmatalis throughout the field season. To assess costs of eavesdropping, I recorded overall growth and in-field defense metrics in the undamaged eavesdroppers. Counter to the original prediction, eavesdropping had little effects on growth and direct defense, while indirect defenses were significantly impacted by neighboring herbivore identity. Specifically, eavesdropping on A. gemmatalis HIPVs resulted in reduced extra- 
floral nectary production in neighboring eavesdropping plants while there was no effect on cyanogenic potential and in-field herbivore resistance. Additionally, neighboring plant identity influenced overall biomass, but not plant defense responses. These results indicate that contextual information provided by HIPVs can differentially affect growth and defense profiles of an eavesdropping plant. These results suggest that HIPVs can be an effective pesticide alternative within intercropping systems. 
Introduction

Eavesdropping is defined as an organism's ability to utilize cues that were intended for another target for their own benefit $[163,164]$. Plants often eavesdrop on herbivore-induced plant volatiles (HIPVs) produced by nearby plants because they accurately portray stress experienced by the plant emitting the volatile signals [165] and potential risk for herbivory, such as neighboring herbivore identity $[30,166]$, the extent of herbivore damage $[61,167]$, and the likelihood of multiple herbivore attack [168170]. As a result of eavesdropping, plants can preemptively allocate resources towards defense that result in enhanced herbivore resistance relative to naïve plants via defense priming (DP) $[26,171,172]$. While eavesdropping and consequent DP are well documented $[17,24,173,174]$, little is known about what factors influence an eavesdropping plant's sensitivity and responses to HIPVs. Since eavesdropping results in enhanced defense profiles upon herbivory, this indicates that DP is an inducible response and that eavesdropping alone is predicted to incur costs $[17,123]$. Eavesdropping occurs in a wide variety of species including sagebrush [156], tobacco [32], black mustard, wild cabbage, and barley $[98,130]$. Therefore, the ability to selectively respond to honest signals is vital to maximize plant fitness, balancing DP with growth and reproduction. However, we collectively know very little as to which environmental factors dictate when an eavesdropping plant utilizes a signal from a neighboring plant.

Neighboring herbivore identity is one factor that may impact eavesdropping costs for plants. Herbivore identity, feeding specialization, feeding guild, feeding 
location, and life history have significant impacts on plant fitness via inducible defenses $[157,175]$. Plants and herbivores are in a constant arms race in which plants increase defenses against herbivores while herbivores adapt and develop new strategies for mitigating plant defense. As a result of this antagonism, herbivores range in feeding efficiency from generalists (by preferentially feeding on a wide range of host plants) to specialists $[175,176]$. In the context of eavesdropping, this variation in types of herbivores present in a system may impact how HIPVs are perceived. Herbivores can differentially influence HIPV blends. For example, HIPVs in Brassica sp. are distinct based on herbivore feeding guild and diet breadth [30] as well as the extent of damage experienced by an emitting plant [61]. Since these variables influence HIPV emissions, I hypothesized that costs of eavesdropping are related to whether it is advantageous for a plant to eavesdrop. In other words, if a plant is exposed to HIPVs produced by a successful herbivore within a system, the costs of eavesdropping should be worthwhile to incur since they outweigh the costs of not being prepared for defense [123]. To date, exposure to herbivore-specific cues reduce both reproductive and somatic growth in neighboring eavesdropping plants $[130,131,177]$. However, these cues are emitted by conspecific plants in response to damage from specialist herbivores that are frequently present in their environments. Thus, the risk of responding to a false signal is fairly low [9]. While this work is informative, it is only the start of our understanding of this phenomenon. Most herbivores fall along the generalist-specialist spectrum and plant communities are often diverse. Therefore, the ability of an eavesdropping plant to 
identity HIPVs based on herbivore specialization on an emitting plant could affect how resources are directed between a specialized or generalized defense response.

Along with neighboring herbivore identity, emitting plant identity plays an important role for signal reliability. In order for an eavesdropper to assess the risk of herbivory, an eavesdropping plant will preferentially respond to HIPVs from a conspecific, rather than a heterospecific, neighbor. Eavesdropping evolved as an unintended, indirect effect from within-plant signaling $[49,50]$. This may explain why neighboring plants may be capable of distinguishing between volatile blends from emitter plants with the same or different genotypes $[178,179]$ as well distinguishing between neighbors of different plant species $[180,181]$. Therefore, eavesdroppers are more likely to utilize conspecific HIPVs since they mirror within-plant signaling [49] and indicate that herbivores are more likely to feed on them [182]. Thus, genetic relatedness between an eavesdropper and a damaged plant may impact the costs of eavesdropping and consequent signal reliability. While eavesdropping plants can respond to cues from both conspecifics and heterospecifics [26, 31, 32], little is known about whether intra-species variation [183-185], such as differences at the genotype $[186,187]$ or cultivar level [188], can influence eavesdropping costs. Domestication resulted in widely varying defense [137] and volatile profiles [189-191] in many species. Therefore, I predicted that cultivar-specific differences may impact eavesdropper responses to HIPVs.

In this study, I wanted to determine if exposure to HIPVs from neighboring conspecifics and heterospecifics reduced growth in neighboring eavesdropping lima 
bean plants. I tested these hypotheses using two lima bean (Phaseolus lunatus) cultivars grown in a common garden. I predicted that eavesdropping on emitter plants exposed to generalist herbivores would provide a larger fitness benefit for eavesdroppers, such as increased reproductive output, compared to those neighboring plants exposed to specialist herbivores. Since specialist herbivores are adapted to overcome chemical and physical defenses more readily compared to generalist herbivores [159, 175, 192, 193], I predicted that it is more advantageous for an eavesdropping plant to allocate resources according to risk. Additionally, I predicted that eavesdroppers would invest more energy in growth and defense when neighboring emitter plants were congeneric cultivars compared to heterogeneric cultivars due to more reliable signaling that mimics withinplant communication $[51,180]$.

Methods

Study site and plants

This study was conducted at Blackacre Conservancy, Louisville KY, USA in the community garden $\left(38^{\circ} 11^{\prime} 33.8^{\prime \prime} \mathrm{N} 85^{\circ} 31^{\prime} 28.3^{\prime \prime W}\right)$ from June 1, 2018-September 1, 2018. The study site was $324 \mathrm{~m}^{2}$ and was enclosed in a mesh fence to exclude mammalian herbivores. 278 pairs of plants comprised of an emitter and an eavesdropper were planted in June 2018. Pairs included either congeneric or heterogeneric cultivars, and each emitter plant was exposed to one of two herbivores (described below),or left as an undamaged control (Fig. 10).

Two P.lunatus cultivars, Henderson and Fordhook 242, were used in this study to assess con- vs. heterospecific differences in eavesdropping. Both Henderson and 
Fordhook seeds were surface sterilized with $15 \%$ bleach for 5 minutes followed by a 5 minute $70 \%$ ethanol. After seeds were rinsed three times with distilled water, one seed per pot was planted in autoclaved Metromix@ 360 potting mix. Both cultivars were raised under greenhouse conditions in the University of Louisville greenhouse before transplanting in the field site as specified in [134].

Herbivores and HIPV manipulations

The herbivores Spodoptera exigua and Anticarsia gemmatalis were chosen for this study because they represent two extremes along the host-range spectrum. In this system, S.exigua acted as our extreme generalist having a broad host range [194] while A.gemmatalis is a legume specialist [195]. Egg masses of both S.exigua and A.gemmatalis were from Benzon biological supply in Carlisle, PA (APHIS permit number P526P-16-0256) and reared on lepidopteran diet (Southland products, Lake Village, Arkansas USA) until they reached $3^{\text {rd }}-4^{\text {th }}$ instar. Before field placement, all herbivores were starved for $\sim 12$ hours to ensure they would damage emitter plants and generate HIPVs $[193,196]$.

When emitter plants were 8 weeks old, I enclosed them in mesh Breather sleeves (Palm tree packaging, Apoka, Florida USA) with caterpillars inside for three days (Fig. 10). I tried to implement a consistent $30 \%$ damage level on plants, which typically generates high levels of HIPVs $[61,101]$ without inhibiting regrowth, insuring that the extent of damage did not act as a confounding variable for generating HIPVs on emitter plants. To simulate naturally-occurring generation times for each herbivore, I introduced herbivores at 3 times throughout the growing season at 30,60, and 90 days after field 
transplanting, generating three separate HIPV pulses. To account for increased biomass over the field season and ensure that damage levels on emitters were consistent and resulted in the similar levels of HIPVs over time, the total number of caterpillars in each emitter bag varied from 15-25 individuals and increased throughout the field season.

\section{Responses measured:}

Growth, biomass, and reproduction measures

Bud, flower, and fruit production were recorded throughout the field season as proximate measures of fitness, while overall growth was quantified as end-of-season biomass. Floral buds were recorded if they exceeded $2 \mathrm{~mm}$ in length and recorded at 7 , 42 , and 71 days after applying herbivores on neighboring emitters. Flower counts included only mature flowers with mature reproductive structures present and were recorded at 45, 75, and 100 days after damaging emitter plants. Pod counts included developing pods at least $2 \mathrm{~mm}$ in length and with developing seeds and were recorded at 49,71 , and 101 days after damaging emitter plants. Biomass measurements were conducted at the end of field season in early September. Plants were dug out of the soil and separated by leaf, stem, pod, and root material in the lab. After washing all roots in water to remove loose soil, all materials were dried at $60^{\circ} \mathrm{C}$ for 24 hours before weighing.

\section{Defense priming quantification}

In order to generate induced defense and assess defense priming in real time, I isolated mature trifoliates and bagged with either an A.gemmatalis, S.exigua individual, or left the trifoliate as an undamaged control after 24 hours of HIPV exposure from 
neighboring emitter plants. After caterpillars were allowed to feed for 24 hours, caterpillars were removed and weighed (Appendix II) and collected trifoliates for processing as outlined below.

To assess how eavesdropping impacts defense induction, I quantified cyanogenic potential in leaves that were collected 24 hours after herbivore damage began on receiver plants to maximize induction profiles $[101,135]$. I focused primarily on cyanogenic potential since it is a prominent defense trait in P.lunatus that is restricted to essential organs to reduce costs of induction $[141,158,197]$. Individual mature leaves were clipped, photographed, and immediately flash-frozen in liquid nitrogen and stored at $-80^{\circ} \mathrm{C}$ prior to lyophilization. A small-scale cyanide colorometric quantification was modified from Gleadow et al. for rapid quantification of total cyanide per sample [142] and used previously in [134]. Briefly, $5 \mathrm{mg}$ of lyophilized tissue was weighed and placed into $2.0 \mathrm{~mL}$ centrifuge tubes and placed on ice. Once all the samples were on ice, $200 \mathrm{uL}$ of cold citrate buffer (0.1 M, pH 5.5-6.5) and an 200 uL vial glass insert (Agilent part number $5183-2090$ ) with $1.0 \mathrm{M} \mathrm{NaOH}$ was placed inside the $2 \mathrm{~mL}$ tube before capping. After a 15 -hour incubation at $37^{\circ} \mathrm{C}, 30 \mathrm{uL}$ of the $0.1 \mathrm{M}$ solution was added in triplicate in a 96 well plate followed by $30 \mathrm{uL} 0.5 \mathrm{M}$ acetic acid, $75 \mathrm{uL}$ of reagent a $\left(5.0 \times 10^{-3} \mathrm{~g} \mathrm{ml}^{-1}\right.$ succinimide, $5.0 \times 10^{-4} \mathrm{~g} \mathrm{ml}^{-1} \mathrm{n}$-chlorosuccinimide) and $30 \mathrm{uL}$ of reagent $\mathrm{b}\left(0.03 \mathrm{~g} \mathrm{~mL}^{-1}\right.$ barbituric acid, 30\% pyridine deionized water). Concentration was calculated by comparing absorbance values at $580 \mathrm{~nm}$ to a standard curve of cyanide ranging from 0$3000 \mu \mathrm{mol}$. $\underline{\text { In-field defense metrics }}$ 
To assess in-field resistance of eavesdroppers, I quantified both direct and indirect measures of defense. For direct defense metrics, I quantified percent leaf area removed (LAR) to assess damage by leaf defoliators. In this assessment, all individual leaves on each plant were binned into specific categories: $0 \%, 0-5 \%, 5-15 \%, 15-30 \%, 30-$ $50 \%, 50-70 \%, 70-90 \%$, and $>90 \%$ damage. Then I calculated the average based on the midpoint within each damage category and the number of leaves to give one LAR value per plant [81].

As one proximate measure for indirect defense, I quantified the total number of extra floral nectary pairs present throughout the field season. Since extra floral nectar is produced to attract ants and the production of extra floral nectaries are plastic in response to herbivore damage $[198,199]$, I predicted that EFN pairs may similarly increase in response to HIPVs exposure.

\section{Data Analysis}

For data collected on multiple dates (e.g., floral counts), I conducted two-factor repeated measures ANOVAs followed by post-hoc Tukey tests on the adjusted means, with herbivore identity and congeneric relatedness as main factors, and with time, eavesdropper plant identity, and emitter plant identity as a covariates (Table 2). Responses that were quantified at one time point (e.g., biomass values and cyanogenic potential), were similarly analyzed via a two-factor ANOVA with herbivore identity and relatedness as main factors, but with eavesdropper and emitter plant identity as covariates in Table 3. Response variables that did not meet normality assumptions were noted in Table 2 and 3 and were transformed appropriately to better meet model 
assumptions. I primarily used the mult comp and Ime4 packages in r-version (3.6.1 (2019-07-05) for running these models and I visualized the results using ggplot2 [83, $144,145]$.

Results

\section{Herbivore identity}

Counter to our predictions, herbivore identity had no effect on growth and reproduction of eavesdropping plants (Table 2, Fig. 11) and I observed no differences for leaf, stem, root, or total biomass (Fig. 12). Additionally, herbivore identity had no effect on direct defenses, but did impact indirect defenses. Exposure to A.gemmatalisgenerated HIPVs resulted in decreased EFN pairs present relative to controls at our final collection point, while being next to S.exigua emitters had no effect on EFN production (Fig. 13a, Table 2). No differences in cyanogenic potential were observed (Fig. 13c, Table 2), and eavesdropping had no effect on in-field herbivore damage (Fig. 13b, Table 2).

\section{Plant identity}

Plant relatedness significantly affected leaf and overall biomass in eavesdropping plants (Table 3). Henderson plants next to congenerics had increased overall biomass relative to Henderson eavesdroppers next to heterogenerics (Fig. 14a) while Fordhook eavesdropper biomass was unaffected by neighboring plant relatedness (Fig. 14a). Interestingly, plant identity had no effect on defense responses or reproductive counts in eavesdropping plants (Table 2). Although I initially tested for relatedness influencing eavesdropping costs, eavesdropping plant identity was a significant covariate for total 
seed counts (Fig. 14b), leaf, and total biomass while emitter plant identity was a significant covariate for bud and flower counts (Table 2).

\section{Discussion}

Here I show that HIPV-mediated effects on eavesdropping plant fitness and defenses are impacted by both herbivore identity and identity of emitter plants. While most measures of plant growth, reproduction, and direct defenses did not vary in response to different herbivores. However, indirect defense investment via EFN production was significantly reduced in plants eavesdropping on A. gemmatalisgenerated HIPVs compared to controls. This difference in response to herbivore-specific HIPVs partially support the predictions that risk assessment influences growth-defense tradeoffs. For example, indirect defenses like EFN production are typically generalized defense responses $[110,200,201]$ and may be less effective against specialists like $A$. gemmatalis (reviewed in[200]). In the presence of herbivore specialists, eavesdropping plants may be better served by investing resources towards reproduction or a specialized indirect defense response such as HIPV emissions to attract parasitoids. Similar tradeoffs occur between foliar herbivore defenses and reproductive output [14, 202].

Neighboring plant relatedness had little effect in this study, though leaf and overall biomass of eavesdropper plants was significantly higher when emitter plants were conspecifics relative to eavesdroppers within a pair. Eavesdropping plants preferentially use volatile cues from neighbors that are genotypically or chemotypically similar to themselves $[60,203]$ rather than heterospecifics $[26,52,178,180,181,204]$. 
In this study, Henderson plants responding to a congeneric emitter had increased overall biomass and seed production, supporting previous work that biomass can act as a reliable proximate fitness measure [205]. To that end, neighboring plant identity may have only impacted biomass since overgrowth is a generalized overcompensation response $[154,156]$ to increase overall fitness. These results suggest that it is adaptive to invest more resources towards growth when exposed to stress signals $[130,156$, 177].

In this study, both herbivore identity and relatedness between emitters and eavesdroppers significantly impacted growth and defenses in eavesdropping plants under field conditions. Future studies should build upon this work to address how eavesdropping costs are impacted by phylogenetic relatedness between emitters and eavesdroppers. To my knowledge, the only other study to assess eavesdropping costs between heterospecific species was conducted between sagebrush and tobacco [32]. Since intercropping is an effective method for reduced pest damage [206], it is imperative to conduct follow-up studies to address how intercropping may impact fitness of other crop plants.

I also found that both emitting plant identity and eavesdropping plant identity impacted both reproductive and vegetative growth. Emitter plant identity influenced bud and flower counts of eavesdropping plants, while eavesdropper plant identity influenced pod production and biomass. Future studies should also further investigate how emitter or eavesdropping plant identity themselves impacts effects of eavesdropping. This work showed that cultivar identity of eavesdropping plants was 
more influential than genetic relatedness between emitters and eavesdroppers for observed shifts in reproduction upon exposure to HIPVs (Appendix II). In lima bean and other plant systems, domestication history impacts cultivar traits such as growth patterns, biomass ratios, and reproductive investment $[207,208]$. Therefore, shifts in defense and morphological traits that result from domestication history $[137,209]$ may explain species-specific growth-defense tradeoffs that influence costs of eavesdropping. The results of this study have potential application for enhancing herbivore resistance in both agricultural and natural settings. 
Table 2: Two-factor repeated measures ANOVA with time, emitter, and eavesdropper plant identity as covariates. Bud counts were log transformed. LAR (Leaf area removed) and EFN (extra-floral nectary pairs) fit a normal distribution. Significant results $(p<0.05)$ are in bold.

\begin{tabular}{|c|c|c|c|c|c|c|c|c|c|c|c|c|c|c|c|}
\hline & \multicolumn{3}{|c|}{$\log$ (Bud counts) } & \multicolumn{3}{|c|}{ Flower counts } & \multicolumn{3}{|c|}{ Pod counts } & \multicolumn{3}{|c|}{ LAR overtime } & \multicolumn{3}{|c|}{ EFN overtime } \\
\hline & Df & F-value & $p$-value & Df & F-value & $p$-value & Df & F-value & $p$-value & Df & F-value & p-value & Df & F-value & $p$-value \\
\hline Herbivore identity & 2,368 & 1.0045 & 0.36723 & 2,328 & 0.786 & 0.4565 & 2,136 & 1.7246 & 0.18212 & 2,386 & 0.1155 & 0.8909 & 2,193 & 3.7161 & 0.02609 \\
\hline Relatedness & 1,368 & 0.002 & 0.9643 & 1,328 & 0.174 & 0.67685 & 1,136 & 0.6916 & 0.40707 & 1,386 & 2.2128 & 0.1377 & 1,193 & 1.5183 & 0.21938 \\
\hline Herbivore identity X Relatedness & s2, 368 & 1.0033 & 0.36767 & 2,328 & 2.5366 & 0.08069 & 2,136 & 0.9456 & 0.39099 & 2,386 & 0.4707 & 0.625 & 2,193 & 2.6468 & 0.07345 \\
\hline Eavesdropper ID & 1,368 & 2.5579 & 0.1106 & 1,328 & 0.9132 & 0.33997 & 1,136 & 4.969 & 0.02745 & 1,386 & 0.366 & 0.5456 & 1,193 & 2.3915 & 0.12364 \\
\hline Emitter ID & 1,368 & 4.9999 & 0.02595 & 1,328 & 6.5145 & 0.01115 & 1,136 & 1.0528 & 0.30669 & 1,386 & 0.1385 & 0.7099 & 1,193 & 2.0433 & 0.15449 \\
\hline Time & 3,368 & 126.6124 & $<0.0001$ & 3,328 & 10.1847 & $<0.0001$ & 3,136 & 23.3036 & $<0.0001$ & 3,386 & 35.7668 & $<<0.0001$ & 3,193 & 259.5075 & $<0.0001$ \\
\hline
\end{tabular}


Table 3: Two-factor ANOVAs with Herbivore identity and relatedness between and emitter and eavesdropping plant as main

factors. Emitter and eavesdropping plant identity were included as covariates. Significant results $(p<0.05)$ are in bold.

\begin{tabular}{|c|c|c|c|c|c|c|c|c|c|}
\hline & \multicolumn{3}{|c|}{$\log$ (Leaf Biomass) } & \multicolumn{3}{|c|}{$\log ($ Stem biomass) } & \multicolumn{3}{|c|}{$\log ($ Root biomass $)$} \\
\hline & Df & F-value & p-value & Df & F-value & p-value & Df & F-value & p-value \\
\hline Herbivore identity & 2,200 & 0.8836 & 0.41491 & 2,200 & 0.3025 & 0.7393 & 2,143 & 0.1795 & 0.8359 \\
\hline Relatedness & 1,200 & 4.2634 & 0.04023 & 1,200 & 2.3268 & 0.1287 & 1,143 & 0.5043 & 0.4788 \\
\hline Herbivore identity X Relatedness & 1,200 & 0.0596 & 0.94218 & 1,200 & 0.1987 & 0.82 & 1,143 & 1.3222 & 0.2698 \\
\hline Eavesdropper ID & 1,200 & 24.0032 & $<0.0001$ & 1,200 & 30.1584 & $<0.0001$ & 1,143 & 0.3119 & 0.5774 \\
\hline \multirow[t]{3}{*}{ Emitter ID } & 1,200 & 0.7796 & 0.37832 & 1,200 & 0.5392 & 0.4636 & 1,143 & 0.2007 & 0.6549 \\
\hline & \multicolumn{3}{|c|}{$\log$ (Total Biomass) } & \multicolumn{3}{|c|}{ Total Seed Production } & \multicolumn{3}{|c|}{ Cyanide induction } \\
\hline & Df & F-value & p-value & Df & F-value & p-value & Df & F-value & p-value \\
\hline Herbivore identity & 2,200 & 0.7203 & 0.48788 & 2,162 & 1.0904 & 0.338532 & 2,38 & 0.0757 & 0.9272 \\
\hline Relatedness & 1,200 & 5.4155 & 0.02096 & 1,162 & 0.4696 & 0.494129 & 1,38 & 0.6877 & 0.4117 \\
\hline Herbivore identity X Relatedness & 1,200 & 0.2106 & 0.81029 & 1,162 & 2.1796 & 0.116395 & 1,38 & 1.1106 & 0.3398 \\
\hline Eavesdropper ID & 1,200 & 19.4483 & $<0.0001$ & 1,162 & 10.0034 & 0.001864 & 1,38 & 2.162 & 0.1493 \\
\hline Emitter ID & 1,200 & 2.1651 & 0.14274 & 2,162 & 0.131 & 0.717848 & 2,38 & 1.1307 & 0.2943 \\
\hline
\end{tabular}




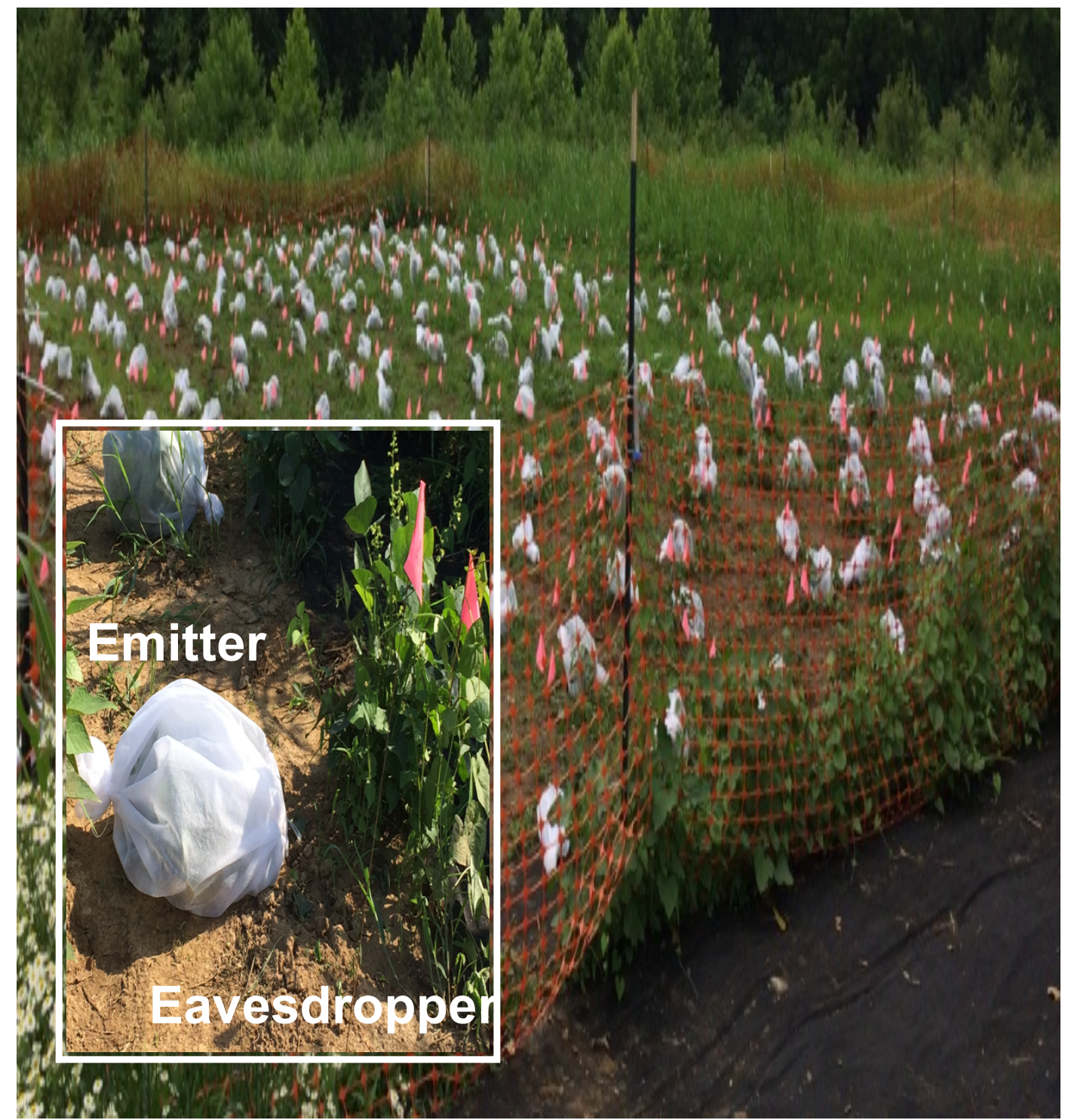

Figure 10: Experimental setup within the field site. I started with 280 pairs of herbivore-damaged (emitter) plants and undamaged neighboring plants (eavesdroppers). Emitter plants were exposed to herbivores at three separate times throughout the field season to generate $\sim 30 \%$ damage over each three-day period. 

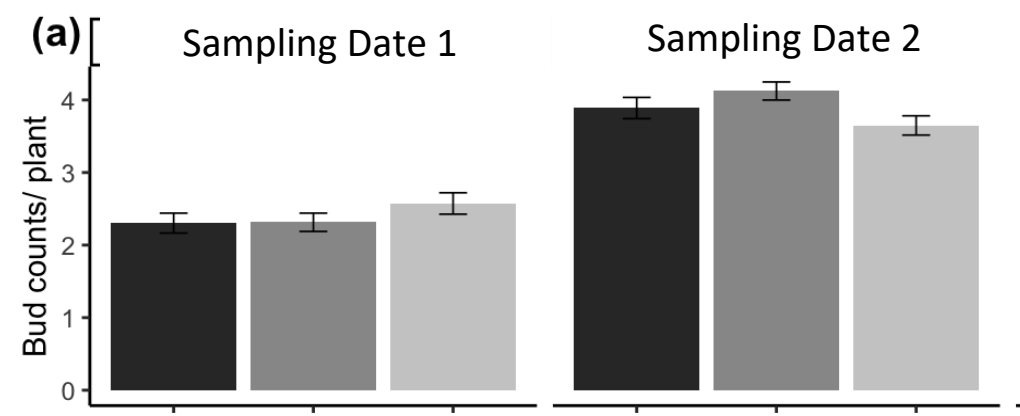

Sampling Date 3
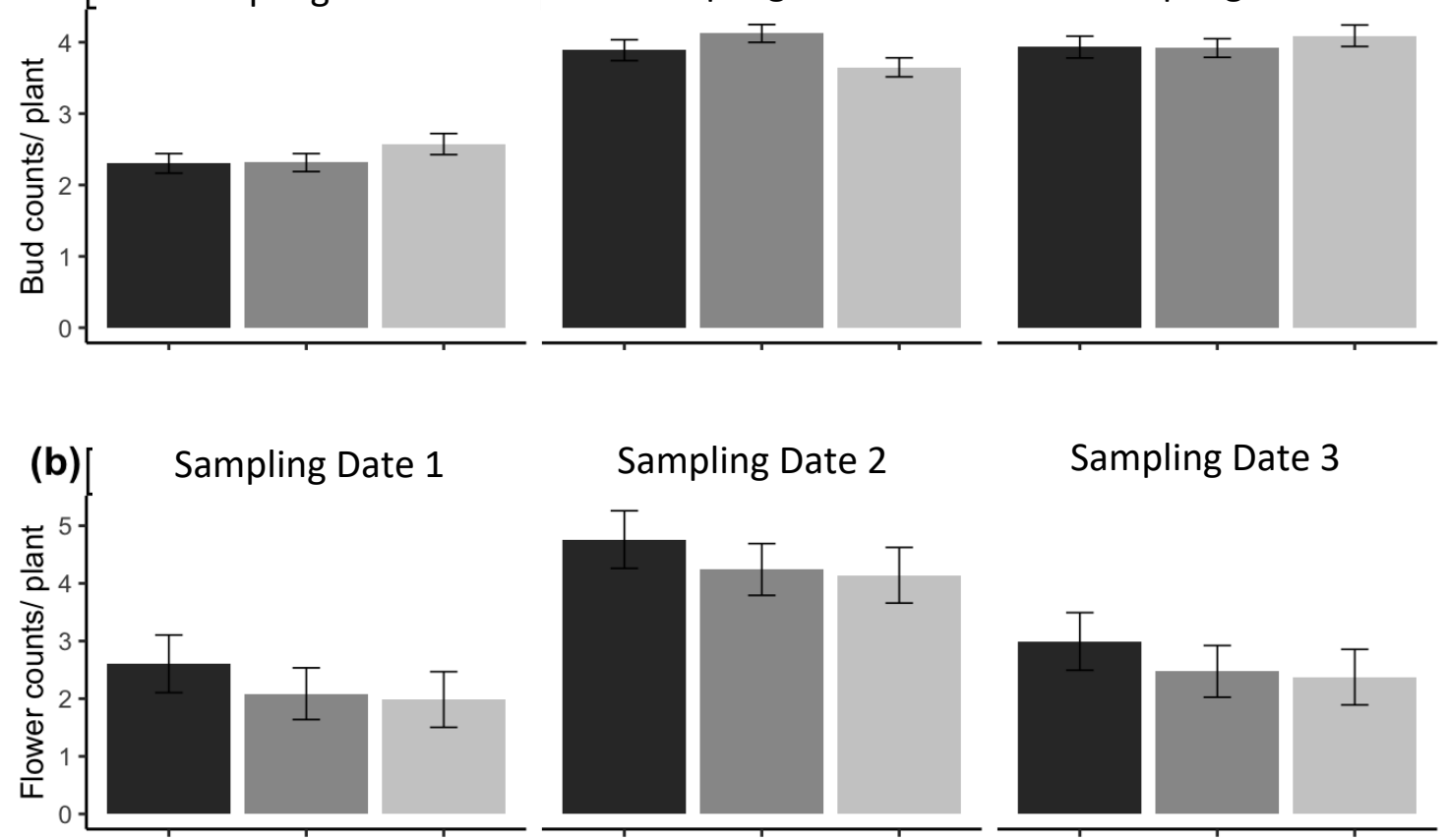

Sampling Date 2

Sampling Date 3
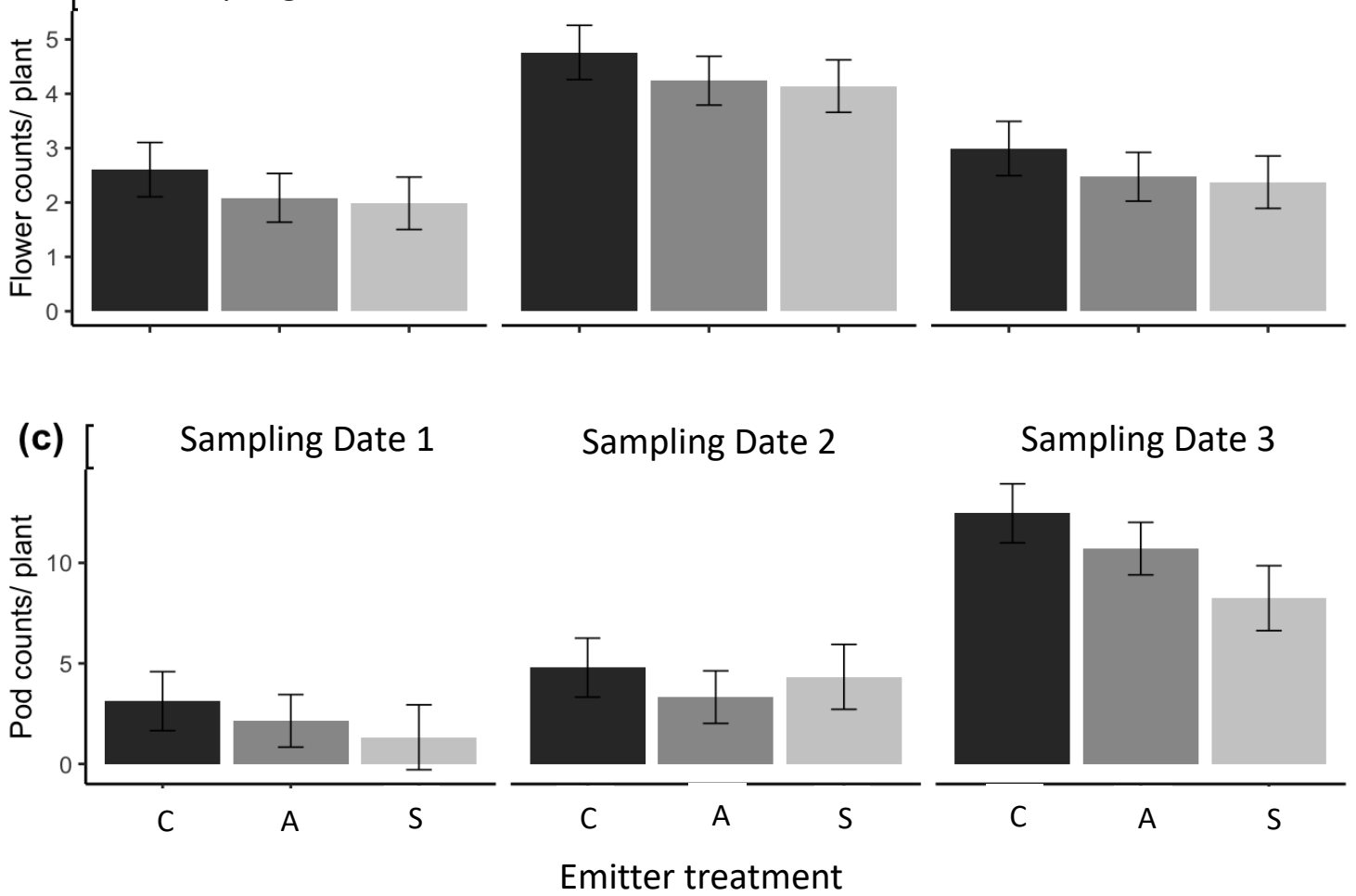

Figure 11: No evidence for herbivore identity effects on adjusted-mean reproductive

output in eavesdropping plants. $\mathrm{C}=$ control, $\mathrm{A}=$ A.gemmatalis, and $\mathrm{S}=\mathrm{S}$. exigua emitter treatment. See methods section for specific collection dates. Error bars indicate +/- 1SE. 

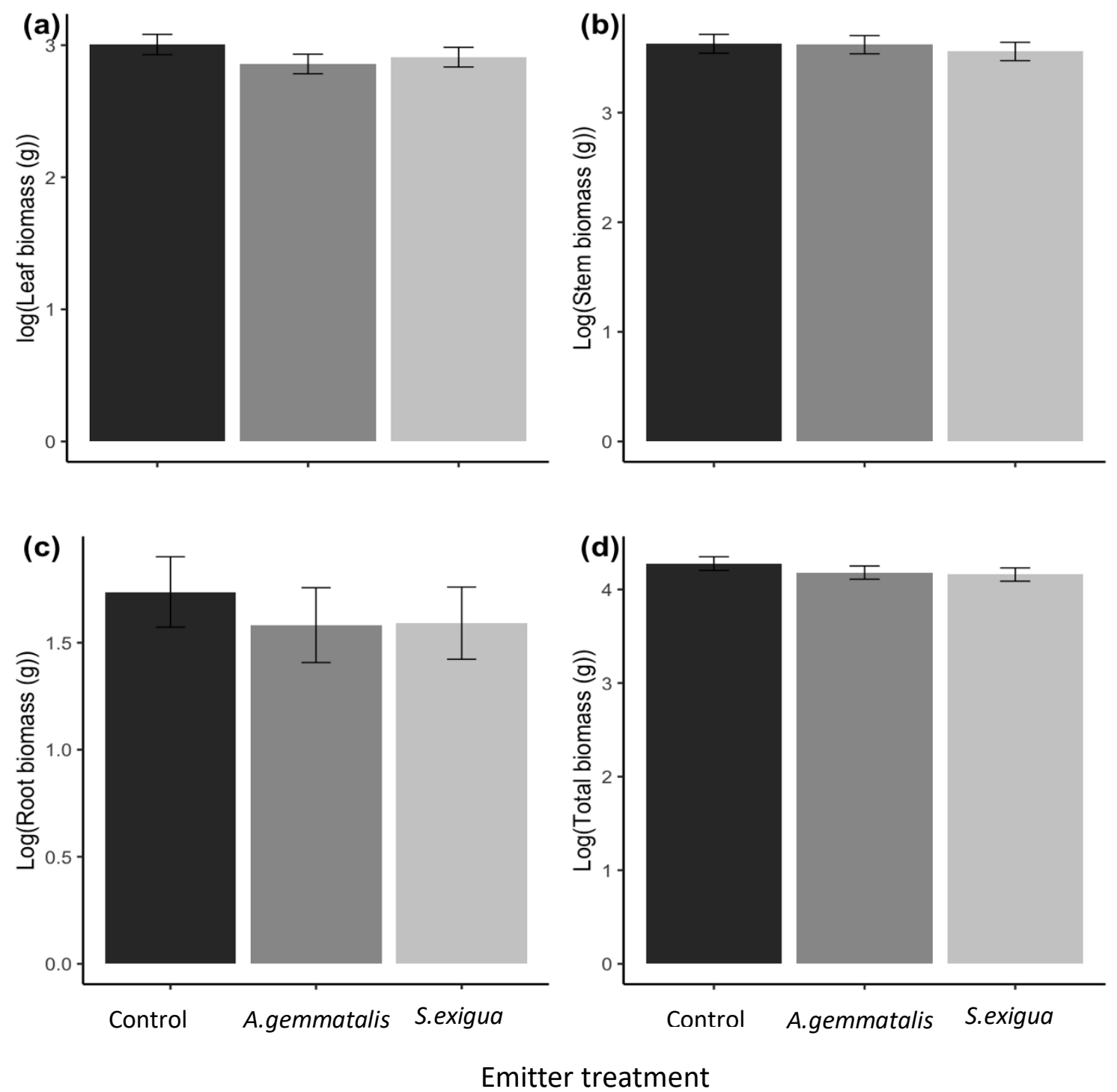

Figure 12: Eavesdropping plant Leaf (a), Stem (b), Root (c), and Total biomass (d) responses to herbivore identity on emitting plants. Error bars indicate +/- 1SE. 

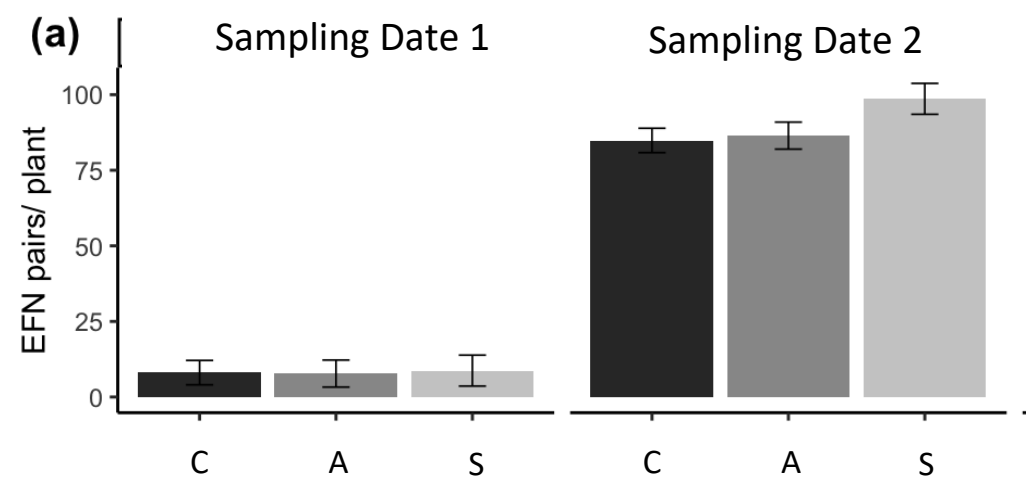

$$
\text { Sampling Date } 3
$$
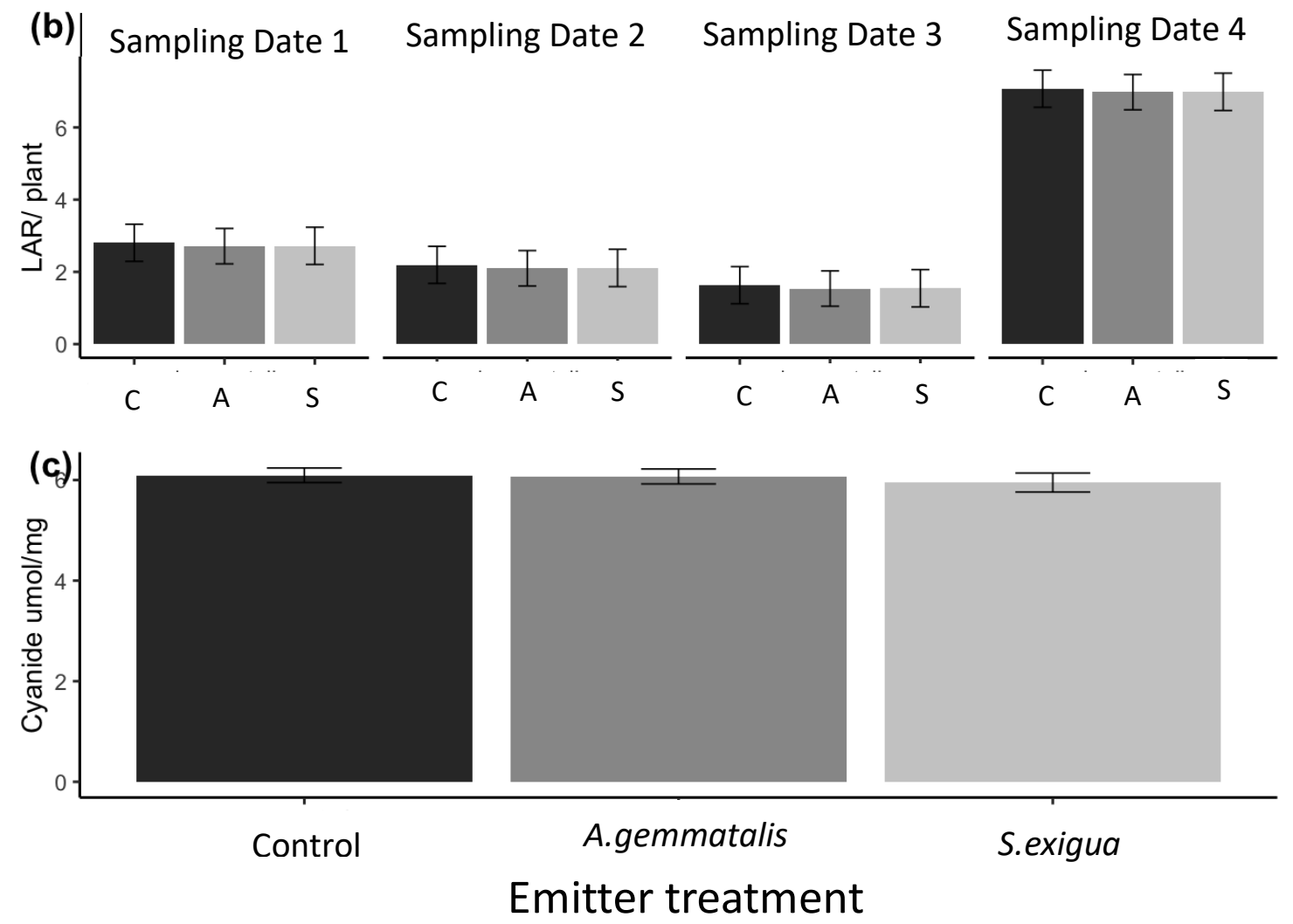

Figure 13: Indirect (a) and direct (b, c) measures of in-field defense as affected by herbivore identity. Letters indicate significant $(p<0.05)$ differences among treatments in Tukey's post-hoc comparisons. $\mathrm{C}=$ control, $\mathrm{A}=$ A.gemmatalis, $\mathrm{S}=\mathrm{S}$. exigua . 

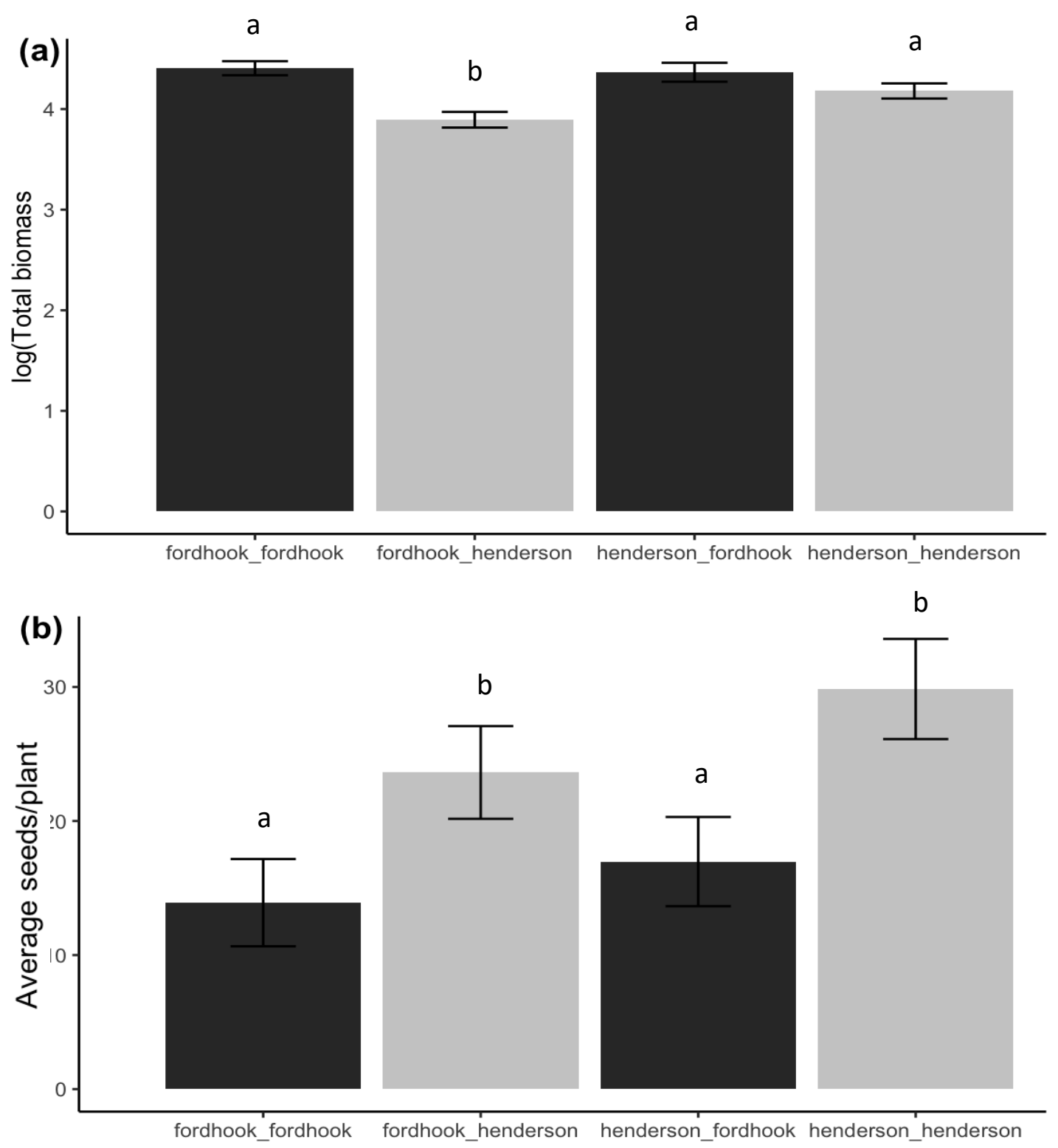

Figure 14. Relatedness between emitters and eavesdroppers impacts overall biomass

(a), while eavesdropper ID affects total seed production (b). Henderson eavesdroppers are in grey bars while Fordhook eavesdroppers are in black bars. Notations on the x-axis indicate emitterID - eavesdropperID. 


\section{CHAPTER V}

\section{MATERNAL EAVESDROPPING HAS NO EFFECTS ON PROGENY PERFORMANCE UNDER FIELD CONDITIONS}

Summary

Transgenerational inheritance occurs when parental stress is so great that it leaves an imprint on the progeny. Transgenerational modifications are a widely documented phenomena within the plant kingdom and occur from exposure to both biotic and abiotic stress. For example, parental exposure to herbivory results in enhanced offspring performance for up to three generations [89]. Transgenerational effects of herbivory have been documented in a wide range of plant species [210-214] in both field and greenhouse settings. Both within and transgenerational modified resistance profiles have been linked to epigenetic modifications [89, 215-219]. To increase herbivore resistance within a plant's lifetime, plants eavesdrop on herbivoreinduced plant volatiles (HIPVs) to prepare for impending herbivore attack since they accurately predict herbivore identity and the degree of damage experienced by a damaged plant. Since eavesdropping results in epigenetic modifications similar to herbivory itself, I wanted to determine if eavesdropping itself acts as a selective pressure and has effects beyond a single generation. Since eavesdropping is widespread within the plant kingdom, I predicted that HIPV-mediated eavesdropping may likewise confer enhanced herbivore resistance in the next generation. Since transgenerational 
benefits may be dependent on in-field herbivore pressure, I expected to observe transgenerational effects earlier in the field season which are lost over time if in-field herbivore pressure is weak.

To test this prediction, I collected seeds from lima bean individuals that were either exposed to HIPVs (eavesdroppers) from the legume generalist Spodoptera exigua or the legume specialist Anticarsia gemmatalis, and compared their performance to seeds of plants directly damaged by S.exigua or A.gemmatalis. I compared seed germination rates, floral phenology, and in-field growth and defenses among offspring plants. Counter to our initial predictions, I found no effect of parental herbivory or eavesdropping on next-generation performance. These results indicate that transgenerational herbivore resistance is difficult to assess under certain field conditions. 


\section{Introduction}

Eavesdropping on herbivore-induced plant volatiles (HIPVs) is essential for mitigating costs of induced defense. When eavesdropping occurs, an eavesdropping plant gains a fitness advantage compared to a naïve plant since a primed individual can respond more quickly and aggressively to potential herbivores [16, 173, 174]. However, priming is also associated with growth costs. For example, goldenrod populations have been shown to incur growth costs in the presence of an insect pheromone [131], while exposure to insect oviposition cues shifted resources away from growth in Brassica sp. while enhancing defenses [130].

Within a single generation, plants can modify their responses to volatile cues [79, 220, 221]. In environments with high herbivore pressure, HIPVs become reliable signals $[9,70]$. When herbivore pressure is strong and consistent enough, the ability to pass benefits of eavesdropping and priming to the next generation should be evolutionarily advantageous [222]. Transgenerational resistance occurs when a parent is exposed to such high levels of stress that this causes an imprint onto the next generation, resulting in enhanced fitness in offspring. In plants, parental exposure to direct herbivory can cause epigenetic changes [215] that result in stronger herbivore-resistance profiles [89, 210, 223]. Additionally, transgenerational effects of herbivory can span multiple generations for both chemical and physical defenses [213, 224]. As a result, herbivory itself acts as a selective pressure that impacts plant population changes overtime. Similarly, in HIPV-mediated eavesdropping, I would predict that eavesdropping alone 
could incur transgenerational fitness effects since resources are diverted towards optimizing growth and defense profiles. This should be especially true in environments with consistently high herbivore presence.

Here, I examine whether HIPV-mediated eavesdropping results in transgenerational effects similar to those seen with direct herbivory. Since HIPVeavesdropping incurred costs in growth but benefits to defenses in the parent generation (F0) [212, 214], I predicted that offspring (F1) of eavesdroppers would have a fitness advantage relative to offspring of non-eavesdropper, especially if herbivores are continually present in the environment . I expected that these transgenerational effects would be weaker than those from F0 plants exposed to direct herbivory. Additionally, I predicted that transgenerational benefits of eavesdropping would be seen in early development [214] and would be lost overtime if in-field herbivore pressure in the F1 generation was low $[225,226]$.

$$
\text { Methods }
$$

\section{Parental treatments}

I evaluated transgenerational effects of herbivory and eavesdropping using seeds collected from lima bean (Phaseolus lunatus) plants from the experiment described in Chapter 4. I had six parental treatments: 1) plants directly damaged by Anticarsia gemmatalis, a specialist herbivore of lima bean, 2) plants directly damaged by Spodoptera exigua, a generalist herbivore of lima bean, 3) plants never exposed to herbivores, 4) plants that were eavesdroppers of plants directly damaged by Anticarsia 
gemmatalis, 5) plants that were eavesdroppers of plants directly damaged by Spodoptera exigua, and 6) plants that were eavesdroppers of non-damaged plants. F1 seed traits

A subset of F1 seeds associated with each parental treatment above was used to characterize transgenerational effects on seed defenses. 10-15 randomly selected seeds from each parental treatment were used for cyanide quantification. These seeds were cryogenically ground and lyophilized. Then cyanogenic potential was quantified as outlined below for foliar quantification and included in Appendix III.

The remainder of seeds from the experiment in $\mathrm{Ch} .4$ were stored at $21^{\circ} \mathrm{C}$ before being surface sterilized with $20 \%$ bleach for 5 minutes followed by $70 \%$ ethanol for an additional 5 minutes. 120 seeds from each parental treatment were sowed in Metromix $360 @$ potting mix and grown under greenhouse conditions. Two weeks after initial sowing, germination rates were calculated in each treatment group.

\section{Common Garden Experiment:}

To evaluate transgenerational effects on F1 offspring, I conducted a commongarden field experiment using germinated plants associated with each parental treatment described above. Once seedlings in the greenhouse were 3 weeks old and started sending out their first trifoliate pair, plants were transplanted into the common garden at Blackacre Conservancy in May 2019 in Louisville, KY (38.2N, -85.7W). For each parental treatment, $60 \mathrm{~F} 1$ plants were initially transplanted to the field site. Plants were placed $1 \mathrm{~m}$ apart in all directions to reduce interplant communication [50, 77]. 
Growth: Plants were transplanted in the field on May 8, 2019 at three weeks of age and grown until October 1. At the end of the field season, all plants were harvested and separated into leaf, stem and root material. All plant materials were washed in water and dried at $60^{\circ} \mathrm{C}$ for 24 hours before weighing.

Reproduction: Plants were monitored weekly throughout the growing season, and reproductive measurements, including first appearance of reproductive structures along with total bud, flower, and pod counts, were recorded 3-4 times throughout the field season. Date of initial bud production was recorded when the first bud reached $1 \mathrm{~mm}$ in length (ensure they were floral buds and not leaf buds), while initial flowers were counted once mature pollen was observed and the sepals were completely open. Fruit initiation date was recorded when pods were $1.5 \mathrm{~mm}$ in length and did not have remnant petals attached to the developing fruit. All reproductive structures (buds, flowers, fruits) were counted 3-4 times throughout the growing season and counts were added together for a single end-of-season measure of total reproduction.

Defense: I evaluated multiple aspects of plant defense, including in-field damage by herbivores and pathogens, (specifically leaf area removed by herbivores, florivory, aphid colonization, and percent pathogen infection), and indirect defenses (specifically extra-floral nectary production and ant colonization).

Estimates of leaf area removed (LAR) by herbivores were used to assess in-field chewing herbivore damage at three times throughout the field season. Estimates from these three dates were then averaged to give a single value of LAR per plant across the entire growing season. Briefly, individual leaves were visually assessed and assigned to a 
damage category: $0 \%, 0-5 \%, 5-15 \%, 15-30 \%, 30-50 \%, 50-70 \%, 70-90 \%$, and $>90 \%$. Then I calculated the average based on the midpoint within each damage category and the number of leaves to give one LAR value per plant [81]. Along with foliar damage, I similarly assessed florivory by assessing the total number of flowers per plant that had visible damage. I monitored aphid presence in the beginning of the field season to assess piercing herbivore resistance. I was only able to track aphid colonization in the beginning of the field season since they switch hosts later on in the field season [129]. Plants were monitored every 5-7 days, with running counts of all aphids present on individual plants including adults and nymphs, until aphids were no longer present in the field site. I characterized pathogen infection using methods similar to LAR assessments when it was present at the beginning of the field season. Leaves were characterized as infected if they had brown spots indicative of leaf anthracnose [227229].

As a measure of indirect defense, extrafloral nectary pair (EFN) counts and ant presence were recorded biweekly on F1 plants. EFN pairs were counted if they were actively recruiting ants and producing nectar. Non-functional or senesced EFN were not included in the total counts. Ant counts were recorded every other week throughout the field season to assess EFN function. Individual EFN and ant counts were summed across the growing season to give a single total value per plant for the MANOVA analysis.

To measure plant defense priming, I applied leaf mechanical wounding with oral secretion treatments to F1 plants on June 6, 2019 (14 days after initial transplanting). For this manipulation, three photosynthetically mature leaves were first wounded by 
crushing $\sim 20 \%$ leaf area with pliers. Leaves were then immediately treated with one of three herbivore oral secretion treatments: Spodoptera exigua + mechanical damage, Anticarsia gemmatalis + mechanical damage, or left as undamaged controls. Oral secretions were collected during the previous field season from caterpillars used to damage emitter plants in chapter 4. Individual caterpillars were gently squeezed with forceps and consequent regurgitant was collected with a sterile pipette. After collection, oral secretions were diluted with distilled water at a 1:3 ratio. Leaves were harvested 24 hours later and flash frozen in liquid nitrogen before being transferred to $-80^{\circ} \mathrm{C}$ freezers. All leaf tissue was cryogenically ground and lyophilized at $-80^{\circ} \mathrm{C}$ prior to chemical analysis.

To assess how eavesdropping impacts defense induction, I quantified cyanogenic potential in leaves that were collected 24 hours after herbivore damage began on receiver plants to maximize induction profiles $[100,135]$. Individual mature leaves were clipped, photographed, and immediately flash-frozen in liquid nitrogen and stored at $80^{\circ} \mathrm{C}$ prior to lyophilization. A small-scale cyanide colorometric quantification was modified from Gleadow et al. for rapid quantification of total cyanide per sample [142] and used previously in this dissertation. Briefly, $5 \mathrm{mg}$ of lyophilized tissue was weighed and placed into $2.0 \mathrm{~mL}$ centrifuge tubes and placed on ice. Once all the samples were on ice, $200 \mathrm{uL}$ of cold citrate buffer (0.1 M, pH 5.5-6.5) and an 200uL vial glass insert (Agilent part number $5183-2090$ ) with $1.0 \mathrm{M} \mathrm{NaOH}$ was placed inside the $2 \mathrm{~mL}$ tube before capping. After a 15 -hour incubation at $37^{\circ} \mathrm{C}, 30 \mathrm{uL}$ of the $0.1 \mathrm{M}$ solution was added in triplicate in a 96 well plate followed by $30 \mathrm{uL} 0.5 \mathrm{M}$ acetic acid, $75 \mathrm{uL}$ of reagent a 
(5.0x10-3g ml-1succinimide, 5.0x10-4g ml-1n-chlorosuccinimide) and 30uL of reagent $\mathrm{b}$ (0.03g mL-1barbituric acid, 30\% pyridine deionized water). Concentration was calculated by comparing absorbance values at $580 \mathrm{~nm}$ to our standard curve of cyanide ranging from $0-3000 \mu \mathrm{mol}$.

In conjunction with quantifying total cyanide, I also assessed total phenolics. To quantify phenolics, $\sim 5 \mathrm{mg}$ of lyophilized leaf tissue was weighed into $1.5 \mathrm{~mL}$ vials and extracted for 24 hours in $1 \% \mathrm{HCl}$ in methanol at $4{ }^{\circ} \mathrm{C}$ [230]. After incubation, chlorophyll was removed with $500 u L$ of chloroform and $350 u L$ water. The aqueous layer was consequently removed for analysis. Total phenolics were assessed by adding $82.5 \mu \mathrm{L}$ of 1:10 Folin-Ciocalteau reagent and $66 \mu \mathrm{L}$ of $7.5 \% \mathrm{Na}_{2} \mathrm{CO}_{3}$ was added into each well of a microplate. After a 30 min incubation period, samples were diluted with $110 \mu \mathrm{L}$ water. Standards were measured at $320 \mathrm{~nm}$ and referenced against a standard curve of chlorogenic acid ranging from $0 \mu \mathrm{g} / \mathrm{mL}-500 \mu \mathrm{g} / \mathrm{mL}$.

\section{Data Analysis}

Seed germination rates, cyanogenic potential of seeds, and cyanogenic potential of non-primed leaves on F1 plants were analyzed using one-factor mixed-model ANOVAs with parental treatment as the main factor. The other response variables described above were not collected until after F1 herbivore-oral-secretion-plusmechanical-damage manipulations were applied. Therefore, floral bud, flower, and fruit onset, and all biomass values were analyzed using two-factor ANOVAs with parental treatment and F1 oral secretion treatment as main factors. 
To more broadly compare parental effects on reproduction and defense, I conducted one-factor MANOVAs with parental treatment as the main factor. Defense was measured by in-field herbivory (LAR), florivory, percent foliar pathogen infection, ant presence, aphid counts, and extra floral nectary pair production. Reproductive investment was quantified by total bud, flower, and fruit counts. All values with the exception of ant presence were averaged across the field season were imputed as a single value for the MANOVA while ant presence recorded over the field season was summed. All analyses were conducted in R (version 3.6.1) [83]. Data that did not meet normality assumptions were log transformed before analyses.

$\underline{\text { Results }}$

Counter to our original hypothesis, I found that parental treatments had no effect on seed traits or on F1 plant growth, reproduction, or defense (Tables 4, 5, 6; Figs. 15-18).

\section{$\underline{\text { Discussion }}$}

Our results were surprising because other studies have found that parental herbivory can significantly modify reproductive development and biomass of offspring $[89,214]$ as well as enhance priming responses in F1 plants when they were exposed to herbivory in the vegetative state of growth [89, 210, 212]. While I initially predicted that costs of eavesdropping would be relatively low to non-existent, I was surprised to find no transgenerational effects of herbivory in this study. Specifically, I predicted that A.gemmatalis herbivory would incur greater fitness effects while S.exigua herbivory would have less of an effect on F1 responses due to feeding differences. A.gemmatalis 
preferentially fed on young leaf and floral tissue while S.exigua fed on mature leaves (personal observations). However, these transgenerational effects may have been overclouded by increased abiotic stress via flooding and low in-field herbivore pressure to maintain transgenerational resistance profiles.

The ability to observe transgenerational costs is dependent on the extent of stress a parent plant experiences in the previous generation as well as the amount of stress experienced by the offspring. Transgenerational effects are often only observed when progeny are exposed to similar stress as their parents either early in development or near the same time as their parents in the previous generation $[89,214]$. However, the F1 plants were not exposed to any significant in-field herbivory. The herbivore oralsecretion treatments were short-lived and were not meant to mimic sustained in-field herbivory. The limited transgenerational effects in our study may be related to low infield herbivore pressure and costs of investment (Appendix III). Throughout the course of in this study, I found relatively low levels of in-field herbivory (Appendix III) before resurgence of the Mexican Bean Beetle E.varivestis in the field site in late July (personal observations). I may have had results similar to previous studies if I had applied live herbivores on plants for extended periods of time, or if herbivore pressure was stronger earlier in the field season [213, 231].

It may also be that transgenerational effects are expressed in traits not measured in our study. For example in Brassica rapa, damage by the herbivores Pieris brassicae and Maemstra brassicae varied in effects across multiple B.rapa generations. Morphological traits like petal area, and chemical responses such as $\beta$-farnesene emissions, were lost 
after a second generation, while increases in nectar volume was retained up to four generations later [232]. Similarly, in Arabidopsis transgenerational effects of salt stress were stronger in offspring from parents directly exposed to salt stress while grandparental and great-grandparental salt stress exposure had limited effects on offspring resistance [225]. Previous work in lima bean has demonstrated that parental exposure to herbivory resulted in transgenerational differences in cyanide production, but only during the seedling stage [212]. I did not begin taking measurements on plant traits until after seedlings were transplanted, and so have missed transgenerational effects that vary temporally.

While no transgenerational costs or benefits of eavesdropping were observed in this study, future studies should address how environmental stress affects transgenerational traits. The summer of 2019 was the wettest summer on record for the area, and resulted in significant flooding within the field site (personal observations). There was significant flooding during our field season in which parts of our field site were covered in water for almost three weeks in June 2019 followed by another flooding incident at the end of July. As a result of this flooding, I lost $~ 1 / 3$ of our plants at the end of the field season. Various studies have shown that flood stress can impede plant growth and herbivore defense due to significant phytohormonal crosstalk [233, 234]. Additionally, flood stress greatly exasperates the effects of herbivory along coastal systems [234, 235]. With increased global warming expected to continuously increase, the next steps for assessing transgenerational affects is to investigate interactive effects between maternal treatment and abiotic stress. 
Table 4: Results of One and Two Factor ANOVAs analyzing differences in F1 growth, reproduction, and defense due to parental treatments (F0) and F1 herbivore priming treatments (mechanical wounding + OS treatments).

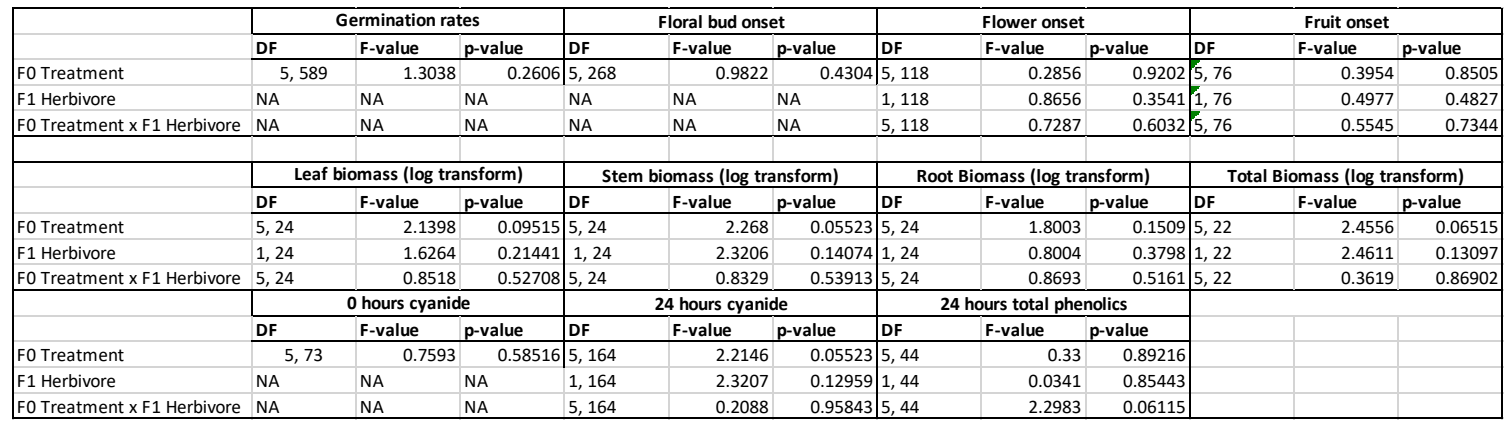


Table 5: One-way MANOVA results for cumulative maternal effects on in-field reproduction and defense. See methods section for factors used in the analysis.

\begin{tabular}{|l|l|r|r|r|}
\hline & df & Wilk's lambda & F & p-value \\
\hline Reproductive output & 5,280 & 0.80599 & 0.99255 & 0.4933 \\
\hline Defense & 5,62 & 0.72067 & 1.0206 & 0.4397 \\
\hline
\end{tabular}




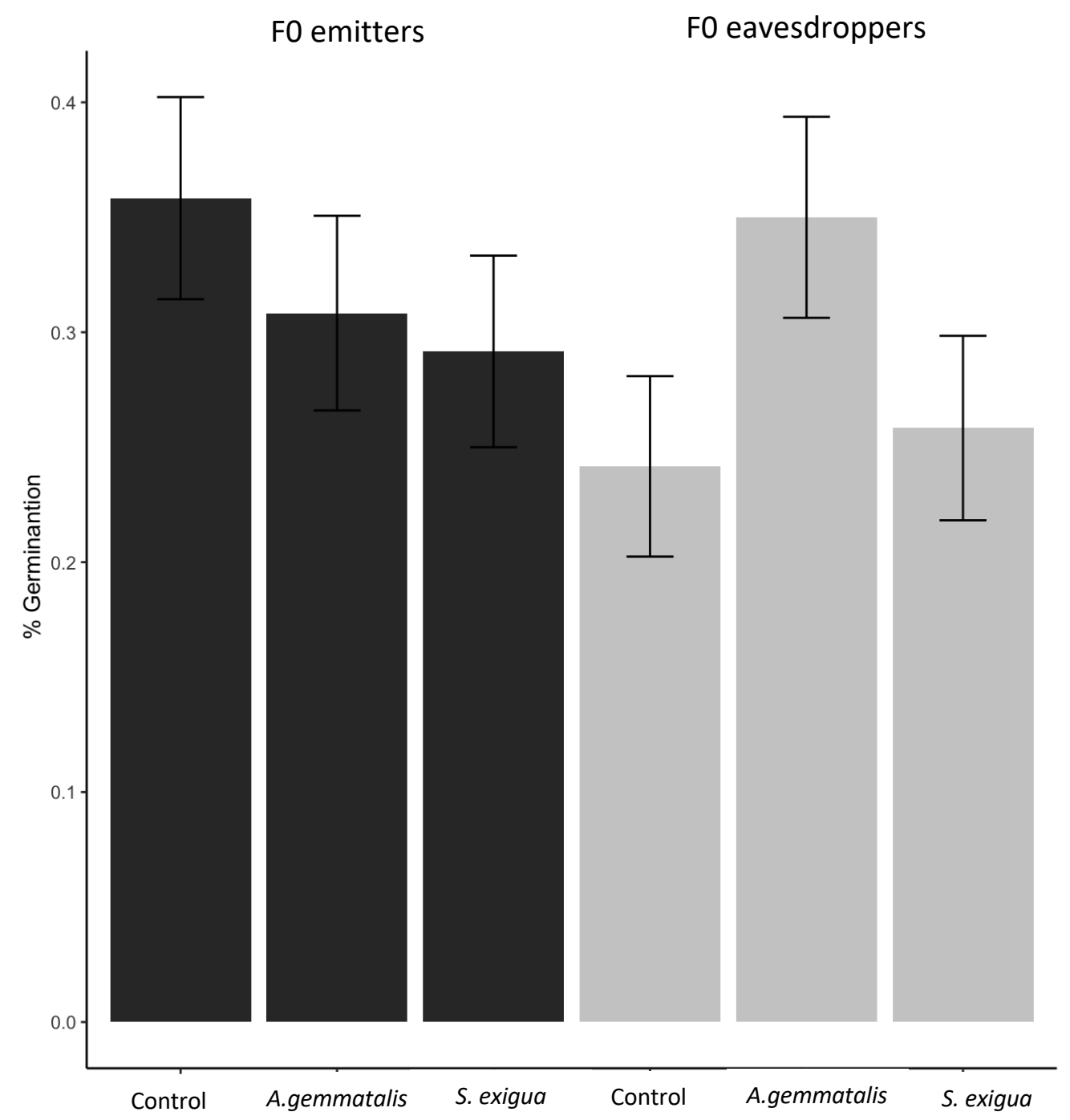

Figure 15. F1 germination rates are unaffected by parental herbivory (F0 Emitter) and HIPV-mediated eavesdropping (F0 eavesdropper). Dark shaded bars are for offspring from parental emitter plants, while light bars are from parental eavesdropping plants. Error bars are +/- $1 \mathrm{SE}$. 
F0 emitters

F0 eavesdroppers
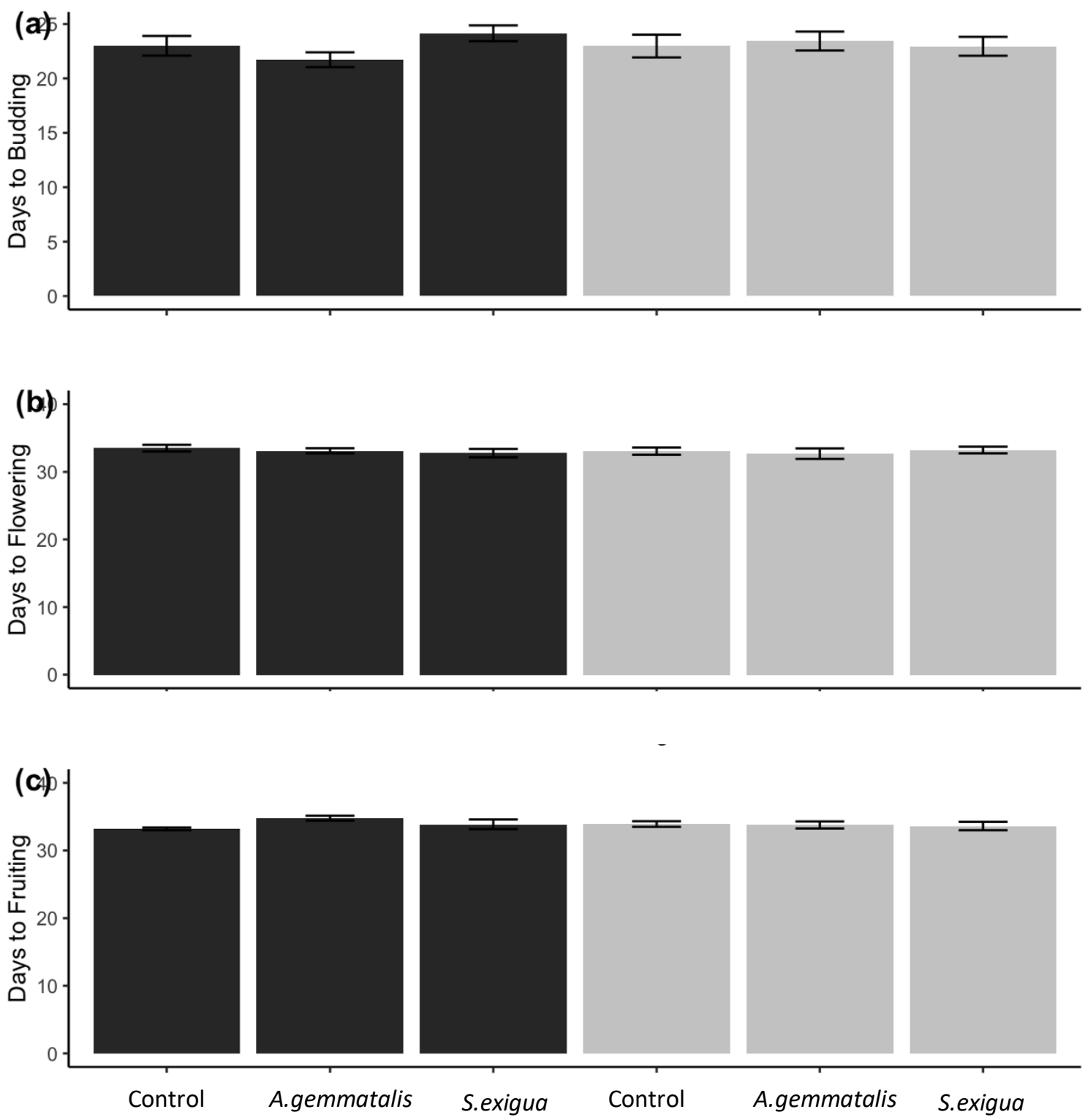

Figure 16: Parental effects on F1 phenology: (A) initial floral bud onset, (B) initial flower onset, and (C) fruit onset. Dark shaded bars are for offspring from parental emitter plants, while light bars are from parental eavesdropping plants. Error bars are +/- 1SE. 
FO

F0 emitters eavesdroppers
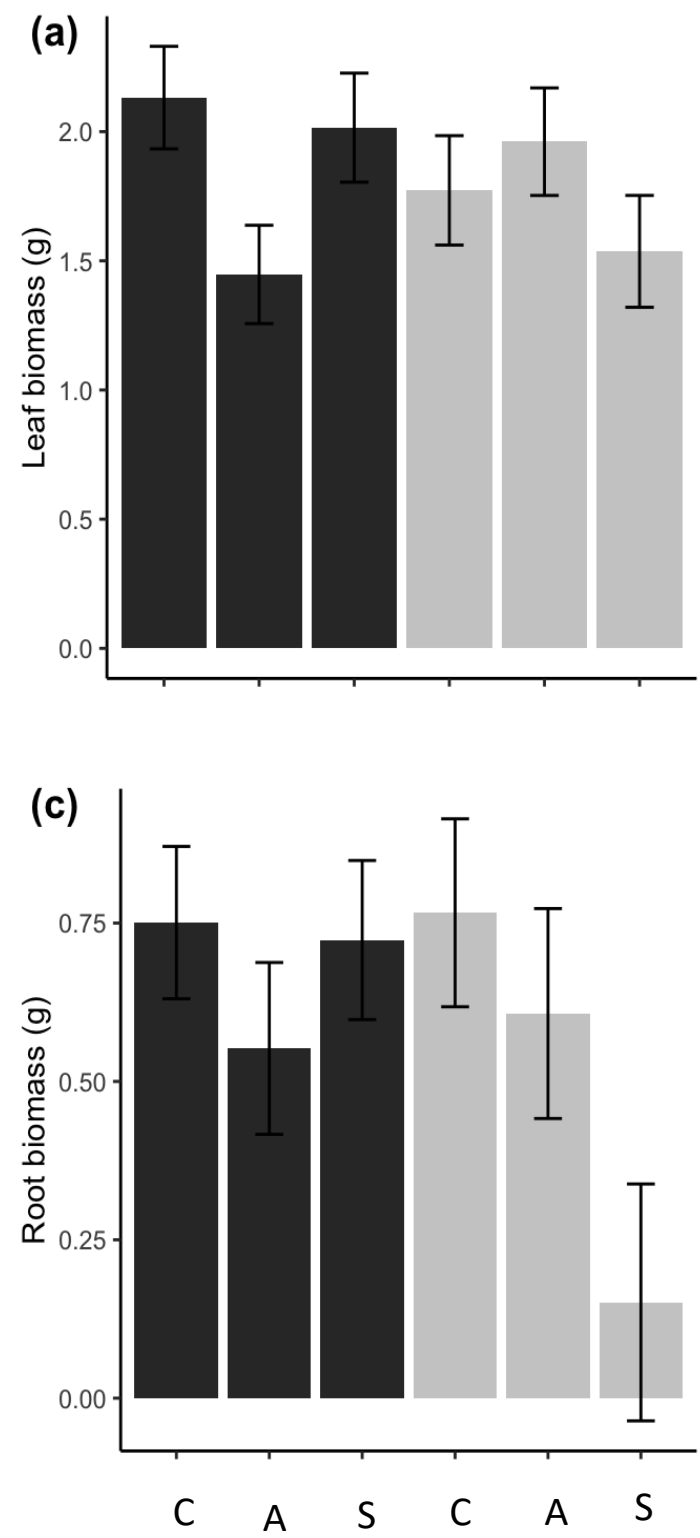

F0

F0 emitters eavesdroppers
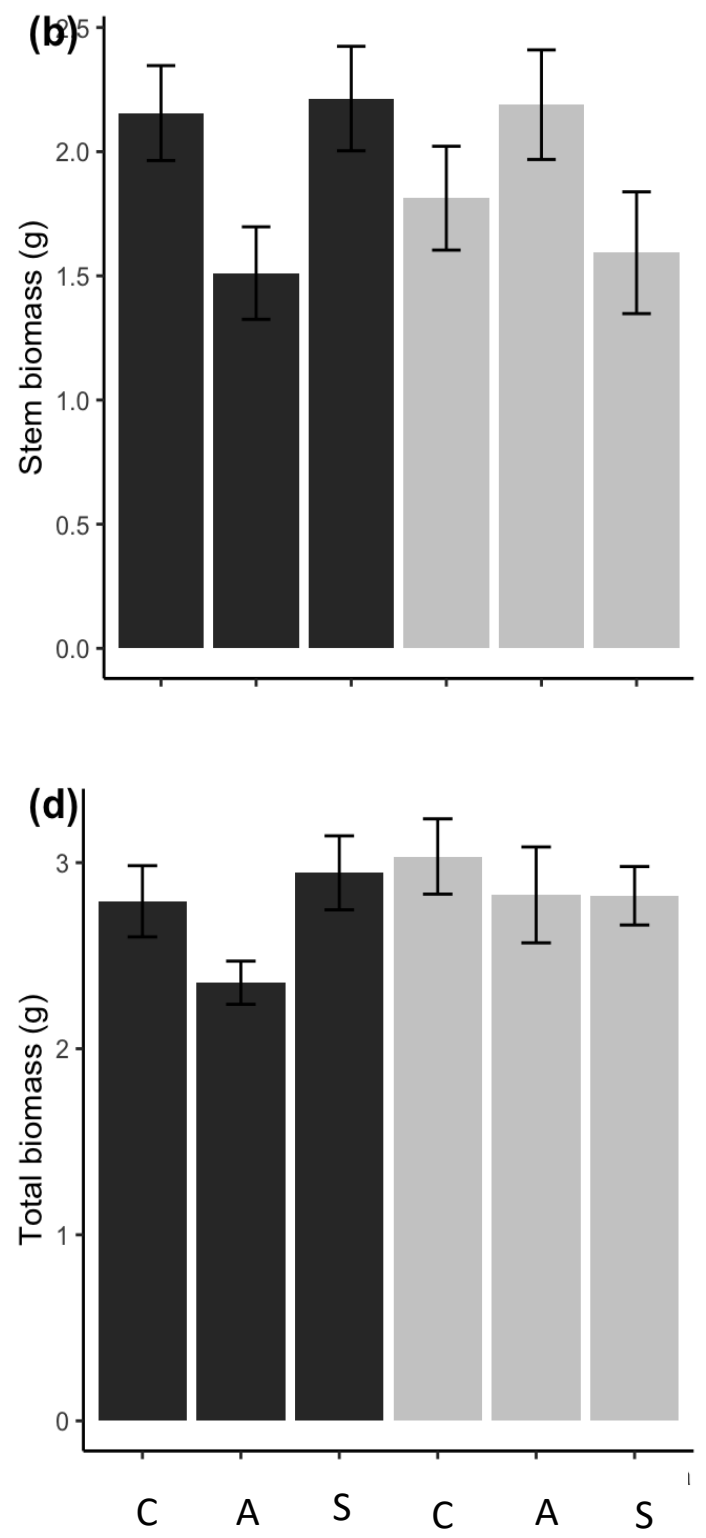

Figure 17. Parental effects on offspring leaf (a), stem (b), root (c), and total biomass

(d). Dark shaded bars are for offspring from parental emitter plants, while light bars are from parental eavesdropping plants On the $\mathrm{x}$-axis, $\mathrm{C}=$ control, $\mathrm{A}=\mathrm{A}$.gemmatalis, and $\mathrm{S}=$ S.exigua. Error bars are +/- 1SE. 
(a)

F0 emitters

F0 eavesdroppers

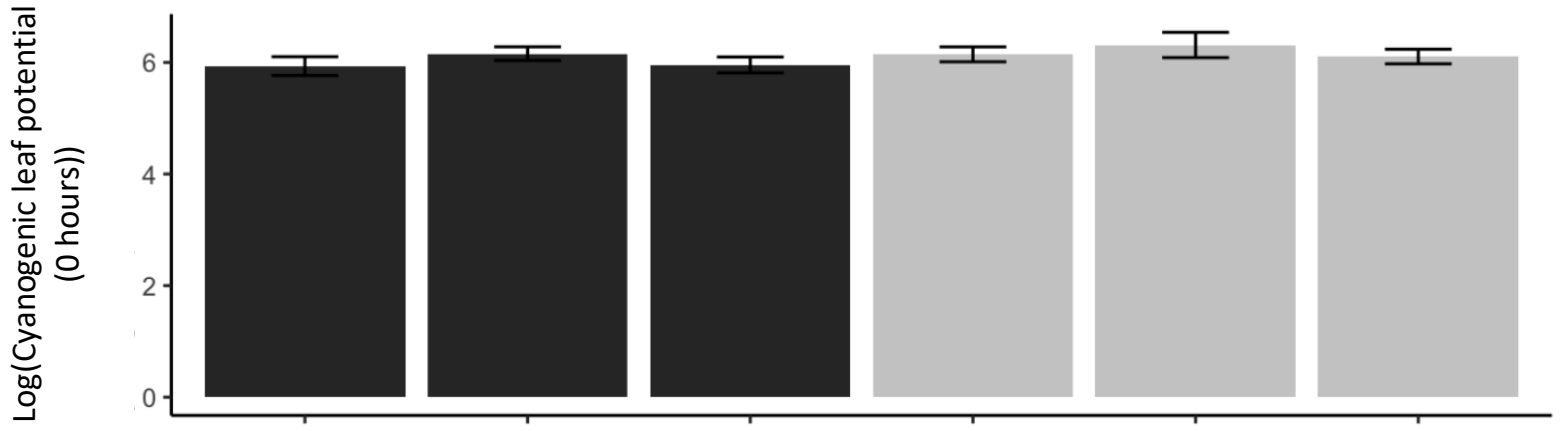

(b)

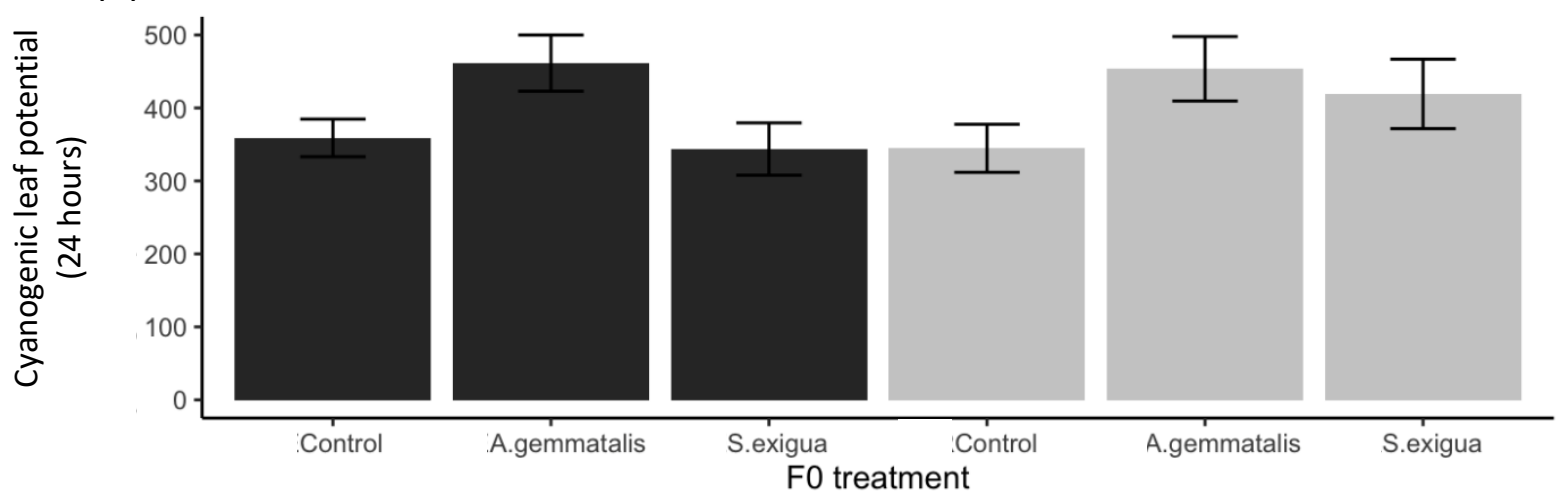

Figure 18: Foliar defenses in F1 plants. Baseline levels of foliar cyanide were quantified

in undamaged leaves (a) and 24 hours after wounding by either A.gemmatalis or

S.exigua (b). Dark shaded bars are for offspring from parental emitter plants, while light bars are from parental eavesdropping plants. Error bars are +/- 1SE. 


\section{CHAPTER VI}

\section{SUMMARY AND FUTURE DIRECTIONS}

Throughout this dissertation, I investigated factors influencing the costs and benefits of ecological eavesdropping and found that exposure to herbivore-induced plant volatiles can impact both growth and defenses. In Ch. 2, I found that exposure to a single herbivore-induced volatile significantly impacted eavesdropper performance, but not always in the way I predicted. While eavesdropping P.lunatus plants had enhanced growth and defense profiles, $C$. annuum experienced costs in vegetative growth and reproductive output.

In Ch. 3, I exposed P.lunatus to the synthetic green-leaf volatile $\mathrm{z} 3 \mathrm{HAC}$ and manipulated duration of exposure. This included a continuous exposure along with two shorter duration periods that simulate naturally occurring herbivory. Since volatile duration acts as a proximate indicator for herbivore presence and severity of herbivory, I predicted that short durations to $\mathrm{z} 3 \mathrm{HAC}$ would result in negligible growth effects and enhance resistance while longer durations would incur significant growth effects. Overall, I found that only a continuous exposure to z3HAC impacted overall growth via increased total biomass relative to other treatment groups, while reproduction and infield defense were unaffected by any z3HAC exposure.

While it is significant to note that exposure to a ubiquitous, herbivore-induced plant volatile can incur significant costs in growth, plants are often exposed to volatile 
blends rather than individual compounds during herbivory that are unique to specific herbivores that reflect their feeding breadth. In chapter 4, I experimentally manipulate herbivore identity using the legume specialist Anticarsia gemmatalis and the generalist crop pest Spodoptera exigua within plant pairs and recorded responses in the undamaged, neighboring eavesdroppers. Direct defense metrics, such as cyanide concentration and in-field herbivore resistance, was unaffected by eavesdropping. However, herbivore-specific effects influenced investment in indirect defense via extra floral nectary production. Eavesdroppers next to plants damaged by A. gemmatalis produced fewer extra floral nectaries relative to controls while plants next to $S$. exigua damaged individuals had no effect. Additionally, I manipulated both eavesdropping plant identity and emitting plant identity using two cultivars of P.lunatus. While emitting plant identity only affected total biomass, eavesdropping costs were significantly impacted by cultivar identity of the eavesdropping plant. Henderson eavesdroppers experienced reproductive costs when next to damaged neighboring plants while Fordhook plants demonstrated an overcompensation response in reproductive output in the presence of HIPVs.

In Ch. 5, I found no evidence for transgenerational effects of HIPV-mediated eavesdropping or direct herbivory. This was surprising to us considering that maternally damaged plants had nearly $30 \%$ damage three separate times during the field season. However, I think maternal effects may have been masked by extreme environmental pressures not related to herbivory. 
Results from this work demonstrate that eavesdropping on herbivore-associated cues along with other environmental factors may impact growth-defense tradeoffs.

While only continuous exposure to z3HAC incurs significant growth effects in lima bean, intermittent exposure to HIPVs resulted in herbivore-specific costs in growth and reproduction. Unique growth effects in the presence of a cue indicates that an eavesdropping plant allocates resources based on perceived risk of herbivory. Specifically, it is more adaptive to divert resources towards defense in the presence of a generalist cue relative to exposure to a cue generated by a specialist herbivore. Additionally, relatively little differences in eavesdropping response upon exposure to HIPVs in chapter 4 indicates that additional factors impact growth-defense tradeoffs. For example, I noticed throughout my dissertation work that in-field herbivore pressure was always the highest towards the end of the field season, typically starting in July with the onset of $E$. varivestis while significant field-site flooding significantly impacted which plants I was able to harvest at the end of the field season in chapter 5 . Therefore, specifically accounting for other environmental variables in conjunction with costs of eavesdropping is important to consider in future work.

While I was unable to quantify differences in volatile blends from damaged plants in this thesis, future work should determine if costs of eavesdropping are specific to unique volatile compounds within HIPVs or the HIPV blends themselves. This work is the first to demonstrate that herbivore identity can impact ecological decision and investment between growth and defense profiles that is heightened by eavesdropping 
plant identity, supporting recent work that has demonstrated that exposure to neighboring HIPVs can modify life history traits [130].

Future Directions

In future studies, it is vital to delineate other potential factors that drive ecological decision-making within HIPV-mediated eavesdropping. A caveat of the experimental design for the fourth chapter is that I did not include a control for possible belowground communication. I inferred that eavesdropping costs resulted from aboveground volatile communication alone. However, previous work has demonstrated that terpenes secreted from roots can impact neighbor plant defenses [236, 237], and that aboveground herbivory can impact belowground root exudates [238]. Therefore, it is plausible that both aboveground and belowground volatile emissions may impact an eavesdropping plant's response.

Additionally, it is vital that I expand upon our current hypothesis describing host range, and how costs of eavesdropping fit into our current understanding of apparency theory [239]. Within this framework, plants invest more heavily in generalized defenses if they are exposed to a wider range of herbivores. Conversely, if they are exposed to a narrower range of herbivores, they may invest more in specialized chemical or physical defense traits. Our results from chapter 4 indicate that exposure to cues from a generalist herbivore result in a generalized response while exposure to specialist cues result in herbivore-specific responses. To expand upon our current findings and determine if this is an overarching trend within eavesdropping, future research should 
expand both the plant species and herbivores used in order to encompass a range of life history traits for both herbivores and eavesdropping plant. 


\section{REFERENCES}

1. Gershenzon, J., Metabolic costs of terpenoid accumulation in higher-plants. Journal of Chemical Ecology, 1994. 20(6): p. 1281-1328.

2. Barton, K.E., Tougher and thornier: general patterns in the induction of physical defence traits. Functional Ecology, 2016. 30(2): p. 181-187.

3. Mauricio, R., Costs of resistance to natural enemies in field populations of the annual plant Arabidopsis thaliana. American Naturalist, 1998. 151(1): p. 20-28.

4. Huot, B., et al., Growth-Defense Tradeoffs in Plants: A Balancing Act to Optimize Fitness. Molecular Plant, 2014. 7(8): p. 1267-1287.

5. Accamando, A.K. and J.T. Cronin, Costs and Benefits of Jasmonic Acid Induced Responses in Soybean. Environmental Entomology, 2012. 41(3): p. 551-561.

6. Feng, Y.J., et al., Costs of Jasmonic Acid Induced Defense in Aboveground and Belowground Parts of Corn (Zea mays L.). Journal of Chemical Ecology, 2012. 38(8): p. 984-991.

7. Cipollini, D., Constitutive expression of methyl jasmonate-inducible responses delays reproduction and constrains fitness responses to nutrients in Arabidopsis thaliana. Evolutionary Ecology, 2010. 24(1): p. 59-68.

8. Moreira, X., et al., Trade-offs between constitutive and induced defences drive geographical and climatic clines in pine chemical defences. Ecology Letters, 2014. 17(5): p. 537-546.

9. Orrock, J.L., et al., Error management in plant allocation to herbivore defense. Trends in Ecology \& Evolution, 2015. 30(8): p. 441-445.

10. Rasmann, S., et al., Trade-off between constitutive and inducible resistance against herbivores is only partially explained by gene expression and glucosinolate production. Journal of Experimental Botany, 2015. 66(9): p. 25272534.

11. Agrawal, A.A., P.M. Gorski, and D.W. Tallamy, Polymorphism in plant defense against herbivory: Constitutive and induced resistance in Cucumis sativus. Journal of Chemical Ecology, 1999. 25(10): p. 2285-2304.

12. Zust, T. and A.A. Agrawal, Trade-Offs Between Plant Growth and Defense Against Insect Herbivory: An Emerging Mechanistic Synthesis. Annu. Rev. Plant Biol, 2017. 68: p. 10.1-10.22.

13. Cipollini, D., C.B. Purrington, and J. Bergelson, Costs of induced responses in plants. Basic and Applied Ecology, 2003. 4(1): p. 79-89.

14. Goheen, J.R., et al., Consequences of herbivory by native ungulates for the reproduction of a savanna tree. Journal of Ecology, 2007. 95(1): p. 129-138.

15. Didiano, T.J., et al., Experimental test of plant defence evolution in four species using long-term rabbit exclosures. Journal of Ecology, 2014. 102(3): p. 584-594.

16. Conrath, U., et al., Priming: Getting ready for battle. Molecular Plant-Microbe Interactions, 2006. 19(10): p. 1062-1071.

17. Frost, C.J., et al., Plant defense priming against herbivores: Getting ready for a different battle. Plant Physiology, 2008. 146(3): p. 818-824. 
18. Appel, H.M. and R.B. Cocroft, Plants respond to leaf vibrations caused by insect herbivore chewing. Oecologia, 2014. 175(4): p. 1257-1266.

19. Hilker, M. and N.E. Fatouros, Plant Responses to Insect Egg Deposition, in Annual Review of Entomology, Vol 60, M.R. Berenbaum, Editor. 2015. p. 493-+.

20. Bandoloy, M., M. Hilker, and A. Stephun, Oviposition by Spodoptera exigua on Nicotiana attenuata primes induced plant defense against larval herbivory. 2015: USA. p. 661-672.

21. Orrock, J.L., Exposure of Unwounded Plants to Chemical Cues Associated with Herbivores Leads to Exposure-Dependent Changes in Subsequent Herbivore Attack. Plos One, 2013. 8(11).

22. Wu, J.Q. and I.T. Baldwin, Herbivory-induced signalling in plants: perception and action. Plant Cell and Environment, 2009. 32(9): p. 1161-1174.

23. Dicke, M. and I.T. Baldwin, The evolutionary context for herbivore-induced plant volatiles: beyond the 'cry for help'. Trends in Plant Science, 2010. 15(3): p. 167175.

24. Scala, A., et al., Green Leaf Volatiles: A Plant's Multifunctional Weapon against Herbivores and Pathogens. International Journal of Molecular Sciences, 2013. 14(9): p. 17781-17811.

25. Peñaflor, M.F.G.V. and J.M.S. Bento, Herbivore-induced plant volatiles to enhance biological control in agriculture. Neotropical Entomology, 2013. 42(4): p. 331-343.

26. Karban, R., L.H. Yang, and K.F. Edwards, Volatile communication between plants that affects herbivory: a meta-analysis. Ecology Letters, 2014. 17(1): p. 44-52.

27. Heil, M., Herbivore- induced plant volatiles: targets, perception and unanswered questions. New Phytologist, 2014. 204(2): p. 297-306.

28. Bricchi, I., et al., Robotic mechanical wounding (MecWorm) versus herbivoreinduced responses: early signaling and volatile emission in Lima bean (Phaseolus lunatus L.). Planta, 2010. 232(3): p. 719-729.

29. Ameye, M., et al., Green leaf volatile production by plants: a meta-analysis. New Phytologist, 2017: p. 1-18.

30. Danner, H., et al., Herbivore-induced plant volatiles accurately predict history of coexistence, diet breadth, and feeding mode of herbivores. New Phytologist, 2017: p. n/a-n/a.

31. Karban, R., et al., Damage-induced resistance in sagebrush: Volatiles are key to intra- and interplant communication. Ecology, 2006. 87(4): p. 922-930.

32. Karban, R. and J. Maron, The fitness consequences of interspecific eavesdropping between plants. Ecology, 2002. 83(5): p. 1209-1213.

33. Meents, A.K., et al., Volatile DMNT systemically induces jasmonate-independent direct anti-herbivore defense in leaves of sweet potato (Ipomoea batatas) plants. Scientific Reports, 2019. 9.

34. Kost, C. and M. Heil, Herbivore-induced plant volatiles induce an indirect defence in neighbouring plants. Journal of Ecology, 2006. 94(3): p. 619-628. 
35. Peng, J., et al., Herbivore-induced volatiles of cabbage (Brassica oleracea) prime defence responses in neighbouring intact plants. Plant Biology, 2011. 13(2): p. 276-284.

36. Choi, H.K., et al., Field Evaluation of the Bacterial Volatile Derivative 3-Pentanol in Priming for Induced Resistance in Pepper. Journal of Chemical Ecology, 2014. 40(8): p. 882-892.

37. Wang, K., et al., Methyl jasmonate induces resistance against Penicillium citrinum in Chinese bayberry by priming of defense responses. Postharvest Biology and Technology, 2014. 98: p. 90-97.

38. $\mathrm{Yu}, \mathrm{C}$., et al., gamma-Aminobutyric acid induces resistance against Penicillium expansum by priming of defence responses in pear fruit. Food Chemistry, 2014. 159: p. 29-37.

39. Song, G.C., H.K. Choi, and C.M. Ryu, Gaseous 3-pentanol primes plant immunity against a bacterial speck pathogen, Pseudomonas syringae pv. tomato via salicylic acid and jasmonic acid-dependent signaling pathways in Arabidopsis. Frontiers in Plant Science, 2015. 6.

40. Kessler, A., et al., Priming of plant defense responses in nature by airborne signaling between Artemisia tridentata and Nicotiana attenuata. Oecologia, 2006. 148(2): p. 280-292.

41. Giron-Calva, P.S., et al., A Role for Volatiles in Intra- and Inter-Plant Interactions in Birch. Journal of Chemical Ecology, 2014. 40(11-12): p. 1203-1211.

42. Wang, M., et al., Effects of the Timing of Herbivory on Plant Defense Induction and Insect Performance in Ribwort Plantain (Plantago lanceolata L.) Depend on Plant Mycorrhizal Status. Journal of Chemical Ecology, 2015. 41(11): p. 10061017.

43. Vicherová, E., et al., Bryophytes can recognize their neighbours through volatile organic compounds. 2020, Nature Reports: Scientific Reports. p. 1-11.

44. Muhlemann, J.K., A. Klempien, and N. Dudareva, Floral volatiles: from biosynthesis to function. Plant Cell and Environment, 2014. 37(8): p. 1936-1949.

45. Mumm, R. and M. Dicke, Variation in natural plant products and the attraction of bodyguards involved in indirect plant defense. Canadian Journal of ZoologyRevue Canadienne De Zoologie, 2010. 88(7): p. 628-667.

46. Farag, M.A., H.M. Zhang, and C.M. Ryu, Dynamic Chemical Communication between Plants and Bacteria through Airborne Signals: Induced Resistance by Bacterial Volatiles. Journal of Chemical Ecology, 2013. 39(7): p. 1007-1018.

47. Frost, C.J., et al., Priming defense genes and metabolites in hybrid poplar by the green leaf volatile cis-3-hexenyl acetate. New Phytologist, 2008. 180(3): p. 722733.

48. Loughrin, J.H., et al., Diurnal cycle of emission of induced volatile terpenoids herbivore-injured cotton plants. Proceedings of the National Academy of Sciences of the United States of America, 1994. 91(25): p. 11836-11840.

49. Frost, C.J., et al., Within-plant signalling via volatiles overcomes vascular constraints on systemic signalling and primes responses against herbivores. Ecology Letters, 2007. 10(6): p. 490-498. 
50. Heil, M. and J.C. Silva Bueno, Within-plant signaling by volatiles leads to induction and priming of an indirect plant defense in nature. Proceedings of the National Academy of Sciences of the United States of America, 2007. 104(13): p. 5467-5472.

51. Heil, M. and R. Karban, Explaining evolution of plant communication by airborne signals. Trends in Ecology \& Evolution, 2010. 25(3): p. 137-144.

52. Pearse, I.S., et al., Interplant volatile signaling in willows: revisiting the original talking trees. Oecologia, 2013. 172(3): p. 869-875.

53. Arimura, G., et al., Herbivore-induced volatiles induce the emission of ethylene in neighboring lima bean plants. Plant Journal, 2002. 29(1): p. 87-98.

54. Farag, M.A., et al., (Z)-3-Hexenol induces defense genes and downstream metabolites in maize. Planta, 2005. 220(6): p. 900-909.

55. Erb, M., et al., Indole is an essential herbivore-induced volatilepriming signal in maize. 2015: Nature communications. p. 1-10.

56. Farag, M.A. and P.W. Paré, C6-Green leaf volatiles trigger local and systemic VOC emissions in tomato. Phytochemistry, 2002. 61(5): p. 545-554.

57. Rodriguez-Saona, C.R., L.E. Rodriguez-Saona, and C.J. Frost, Herbivore-Induced Volatiles in the Perennial Shrub, Vaccinium corymbosum, and Their Role in Interbranch Signaling. Journal of Chemical Ecology, 2009. 35(2): p. 163-175.

58. Sarai Giron-Calva, P., J. Molina-Torres, and M. Heil, Volatile Dose and Exposure Time Impact Perception in Neighboring Plants. Journal of Chemical Ecology, 2012. 38(2): p. 226-228.

59. Arimura, G.I., et al., Effects of feeding Spodoptera littoralis on lima bean leaves: IV. Diurnal and nocturnal damage differentially initiate plant volatile emission. Plant Physiology, 2008. 146(3): p. 965-973.

60. Arimura, G., K. Matsui, and J. Takabayashi, Chemical and Molecular Ecology of Herbivore-Induced Plant Volatiles: Proximate Factors and Their Ultimate Functions. Plant and Cell Physiology, 2009. 50(5): p. 911-923.

61. Copolovici, L., et al., Volatile Emissions from Alnus glutionosa Induced by Herbivory are Quantitatively Related to the Extent of Damage. Journal of Chemical Ecology, 2011. 37(1): p. 18-28.

62. Boggia, L., et al., Direct Contact-Sorptive Tape Extraction coupled with Gas Chromatography-Mass Spectrometry to reveal volatile topographical dynamics of lima bean (Phaseolus lunatus L.) upon herbivory by Spodoptera littoralis Boisd. BMC Plant Biology, 2015. 15(1): p. 1-13.

63. Engelberth, J., et al., Airborne signals prime plants against insect herbivore attack. Proceedings of the National Academy of Sciences of the United States of America, 2004. 101(6): p. 1781-1785.

64. Paschold, A., R. Halitschke, and I.T. Baldwin, Using 'mute' plants to translate volatile signals. Plant Journal, 2006. 45(2): p. 275-291.

65. Matsui, K., et al., Differential Metabolisms of Green Leaf Volatiles in Injured and Intact Parts of a Wounded Leaf Meet Distinct Ecophysiological Requirements. Plos One, 2012. 7(4). 
66. Pastor, V., et al., Preparing to fight back: generation and storage of priming compounds. Frontiers in Plant Science, 2014. 5.

67. D'Auria, J.C., et al., Characterization of a BAHD acyltransferase responsible for producing the green leaf volatile (Z)-3-hexen-1-yl acetate in Arabidopsis thaliana. Plant Journal, 2007. 49(2): p. 194-207.

68. McCormick, A.C., et al., Herbivore-induced volatile emission in black poplar: regulation and role in attracting herbivore enemies. Plant Cell and Environment, 2014. 37(8): p. 1909-1923.

69. Cipollini, D. and a.M. Heil, Costs and benefits of induced resistance to herbivores and pathogens in plants. CAB International, 2010. 5: p. 1-25.

70. Douma, J.C., et al., When does it pay off to prime for defense? A modeling analysis. New Phytologist, 2017. 216(3): p. 782-797.

71. Song, G.C. and C.M. Ryu, Two Volatile Organic Compounds Trigger Plant SelfDefense against a Bacterial Pathogen and a Sucking Insect in Cucumber under Open Field Conditions. International Journal of Molecular Sciences, 2013. 14(5): p. 9803-9819.

72. van Hulten, M., et al., Costs and benefits of priming for defense in Arabidopsis. Proceedings of the National Academy of Sciences of the United States of America, 2006. 103(14): p. 5602-5607.

73. Stenberg, J.A., et al., Optimizing Crops for Biocontrol of Pests and Disease. Trends in Plant Science, 2015. 20(11): p. 698-712.

74. Poelman, E.H., et al., Hyperparasitoids Use Herbivore-Induced Plant Volatiles to Locate Their Parasitoid Host. Plos Biology, 2012. 10(11).

75. von Merey, G., et al., Dispensing synthetic green leaf volatiles in maize fields increases the release of sesquiterpenes by the plants, but has little effect on the attraction of pest and beneficial insects. Phytochemistry, 2011. 72(14-15): p. 1838-1847.

76. Ballhorn, D.J., et al., Quantitative Variability of Direct Chemical Defense in Primary and Secondary Leaves of Lima Bean (Phaseolus lunatus) and Consequences for a Natural Herbivore. Journal of Chemical Ecology, 2008. 34(10): p. 1298-1301.

77. Heil, M. and R.M. Adame-Alvarez, Short signalling distances make plant communication a soliloquy. Biology Letters, 2010. 6(6): p. 843-845.

78. Engelberth, J., et al., Insect elicitors and exposure to green leafy volatiles differentially upregulate major octadecanoids and transcripts of 12-oxo phytodienoic acid reductases in Zea mays. Molecular Plant-Microbe Interactions, 2007. 20(6): p. 707-716.

79. Shiojiri, K., et al., Intermittent exposure to traces of green leaf volatiles triggers a plant response. Scientific Reports, 2012. 2.

80. Ameye, M., et al., Priming of Wheat with the Green Leaf Volatile Z-3-Hexenyl Acetate Enhances Defense against Fusarium graminearum But Boosts Deoxynivalenol Production. Plant Physiology, 2015. 167(4): p. 1671-1684. 
81. Frost, C.J. and M.D. Hunter, Insect canopy herbivory and frass deposition affect soil nutrient dynamics and export in oak mesocosms. Ecology, 2004. 85(12): p. 3335-3347.

82. Frost, C.J. and M.D. Hunter, Herbivore-induced shifts in carbon and nitrogen allocation in red oak seedlings. New Phytologist, 2008. 178(4): p. 835-845.

83. team, R.C., $R$ : A language and environment for statistical computing, R.f.f.s. computing, Editor. 2020: Vienna, Austria.

84. Koricheva, J., Meta-analysis of sources of variation in fitness costs of plant antiherbivore defenses. Ecology, 2002. 83(1): p. 176-190.

85. Hilker, M.e.a., Priming and memory of stress responses inorganisms lacking a nervous system. 2015, Cambridge Philosophical Society: Biological Reviews. p. 116.

86. Pilson, D. and K.L. Decker, Compensation for herbivory in wild sunflower: Response to simulated damage by the head-clipping weevil. Ecology, 2002. 83(11): p. 3097-3107.

87. Hughes, P.W., Between semelparity and iteroparity: Empirical evidence for a continuum of modes of parity. Ecology and Evolution, 2017. 7(20): p. 8232-8261.

88. Miller, T.E.X., B. Tenhumberg, and S.M. Louda, Herbivore-mediated ecological costs of reproduction shape the life history of an iteroparous plant. American Naturalist, 2008. 171(2): p. 141-149.

89. Rasmann, S., et al., Herbivory in the previous generation primes plants for enhanced insect resistance. Plant Physiology, 2012. 158(2): p. 854-863.

90. Blue, E., et al., Differential effects of type and quantity of leaf damage on growth, reproduction and defence of lima bean (Phaseolus lunatus L.). Plant Biology, 2015. 17(3): p. 712-719.

91. Walters, D.R., et al., Priming for plant defense in barley provides benefits only under high disease pressure. Physiological and Molecular Plant Pathology, 2008. 73(4-5): p. 95-100.

92. Choh, Y. and J. Takabayashi, Herbivore-induced extrafloral nectar production in lima bean plants enhanced by previous exposure to volatiles from infested conspecifics. Journal of Chemical Ecology, 2006. 32(9): p. 2073-2077.

93. Acevedo, F.E., et al., Fall Armyworm-Associated Gut Bacteria Modulate Plant Defense Responses. Molecular Plant-Microbe Interactions, 2017. 30(2): p. 127137.

94. Machado, R.A.R., et al., Leaf-herbivore attack reduces carbon reserves and regrowth from the roots via jasmonate and auxin signaling. New Phytologist, 2013. 200(4): p. 1234-1246.

95. Eichenberg, D., et al., Trade-offs between physical and chemical carbon-based leaf defence: of intraspecific variation and trait evolution. Journal of Ecology, 2015. 103(6): p. 1667-1679.

96. Gomez, S., et al., Methyl jasmonate elicits rapid changes in carbon and nitrogen dynamics in tomato. New Phytologist, 2010. 188(3): p. 835-844. 
97. Schweiger, R., et al., Interactions between the jasmonic and salicylic acid pathway modulate the plant metabolome and affect herbivores of different feeding types. Plant Cell and Environment, 2014. 37(7): p. 1574-1585.

98. Ninkovic, V., Volatile communication between barley plants affects biomass allocation. Journal of Experimental Botany, 2003. 54(389): p. 1931-1939.

99. Lu, H.B., et al., Nematicidal Activity of trans-2-Hexenal against Southern RootKnot Nematode (Meloidogyne incognita) on Tomato Plants. Journal of Agricultural and Food Chemistry, 2017. 65(3): p. 544-550.

100. Lucas-Barbosa, D., Integrating Studies on Plant-Pollinator and Plant-Herbivore Interactions. Trends in Plant Science, 2016. 21(2): p. 125-133.

101. Agrawal, A.A., S.Y. Strauss, and M.J. Stout, Costs of induced responses and tolerance to herbivory in male and female fitness components of wild radish. Evolution, 1999. 53(4): p. 1093-1104.

102. Pashalidou, F.G., et al., Phenotypic plasticity of plant response to herbivore eggs: effects on resistance to caterpillars and plant development. Ecology, 2013. 94(3): p. 702-713.

103. Agrawal, A.A., Overcompensation of plants in response to herbivory and the byproduct benefits of mutualism. Trends in Plant Science, 2000. 5(7): p. 309-313.

104. Agrawal, A.A., Induced responses to herbivory in wild radish: Effects on several herbivores and plant fitness. Ecology, 1999. 80(5): p. 1713-1723.

105. Heath, J.J., et al., Exploring plant defense theory in tall goldenrod, Solidago altissima. New Phytologist, 2014. 202(4): p. 1357-1370.

106. Halpern, S.L., L.S. Adler, and M. Wink, Leaf herbivory and drought stress affect floral attractive and defensive traits in Nicotiana quadrivalvis. Oecologia, 2010. 163(4): p. 961-971.

107. Hoffmeister, M. and R.R. Junker, Herbivory-induced changes in the olfactory and visual display of flowers and extrafloral nectaries affect pollinator behavior. Evolutionary Ecology, 2016: p. 1-16.

108. Kessler, A., R. Halitschke, and K. Poveda, Herbivory-mediated pollinator limitation: negative impacts of induced volatiles on plant-pollinator interactions. Ecology, 2011. 92(9): p. 1769-1780.

109. Kessler, A. and I.T. Baldwin, Defensive function of herbivore-induced plant volatile emissions in nature. Science, 2001. 291(5511): p. 2141-2144.

110. Heil, M., Indirect defence via tritrophic interactions. New Phytologist, 2008. 178(1): p. 41-61.

111. Peñaflor, M.F.G.V., et al., Effects of single and multiple herbivory by host and non-host caterpillars on the attractiveness of herbivore-induced volatiles of sugarcane to the generalist parasitoid Cotesia flavipes. Entomologia Experimentalis et Applicata, 2017. 165(1): p. 83-93.

112. Penaflor, M. and J.M.S. Bento, Herbivore-Induced Plant Volatiles to Enhance Biological Control in Agriculture. Neotropical Entomology, 2013. 42(4): p. 331343. 
113. Lucchi, A., et al., Using herbivore-induced plant volatiles to attract lacewings, hoverflies and parasitoid wasps in vineyards: achievements and constraints. Bulletin of Insectology, 2017. 70(2): p. 273-282.

114. Webster, B., et al., Identification of volatile compounds used in host location by the black bean aphid, Aphis fabae. Journal of Chemical Ecology, 2008. 34(9): p. 1153-1161.

115. Wei, J., et al., Reciprocal crosstalk between jasmonate and salicylate defencesignalling pathways modulates plant volatile emission and herbivore hostselection behaviour. Journal of Experimental Botany, 2014. 65(12): p. 3289-3298.

116. Holopainen, J.K. and J. Gershenzon, Multiple stress factors and the emission of plant VOCs. Trends in Plant Science, 2010. 15(3): p. 176-184.

117. Karban, R., Tradeoff between resistance induced by volatile communication and over-topping vertical growth. Plant Signaling \& Behavior, 2017: p. 00-00.

118. Caparrotta, S., et al., Induction of priming by salt stress in neighboring plants. Environmental and Experimental Botany, 2018. 147: p. 261-270.

119. Arimura, G., A. Muroi, and M. Nishihara, Plant-plant-plant communications, mediated by (E)-beta-ocimene emitted from transgenic tobacco plants, prime indirect defense responses of lima beans. Journal of Plant Interactions, 2012. 7(3): p. 193-196.

120. Hu, L.F., M. Ye, and M. Erb, Integration of two herbivore-induced plant volatiles results in synergistic effects on plant defence and resistance. Plant Cell and Environment, 2019. 42(3): p. 959-971.

121. Hilker, M., et al., Priming and memory of stress responses in organisms lacking a nervous system. Biological Reviews, 2016. 91(4): p. 1118-1133.

122. Choh, Y. and J. Takabayashi, Herbivore-induced plant volatiles prime two indirect defences in lima bean. Trends in Acarology, 2010: p. 255-258.

123. Martinez-Medina, A., et al., Recognizing Plant Defense Priming. Trends in Plant Science, 2016. 21(10): p. 818-822.

124. Ameye, M., et al., Green leaf volatile production by plants: a meta-analysis. New Phytologist, 2018. 220(3): p. 666-683.

125. Nottingham, L.B., et al., Natural History, Ecology, and Management of the Mexican Bean Beetle (Coleoptera: Coccinellidae) in the United States. Journal of Integrated Pest Management, 2016. 7(1).

126. Greenberg, S.M., et al., Feeding and life history of Spodoptera exigua (Lepidoptera : Noctuidae) on different host plants. Annals of the Entomological Society of America, 2001. 94(4): p. 566-575.

127. Azidah, A.A. and M. Sofian-Azirun, Life history of Spodoptera exigua (Lepidoptera : Noctuidae) on various host plants. Bulletin of Entomological Research, 2006. 96(6): p. 613-618.

128. Farahani, S., B. Naseri, and A.A. Talebi, Comparative Life Table Parameters of the Beet Armyworm, Spodoptera exigua (Hubner) (Lepidoptera, Noctuidae) on Five Host Plants. Journal of the Entomological Research Society, 2011. 13: p. 91-101. 
129. Sandrock, C., J. Razmjou, and C. Vorburger, Climate effects on life cycle variation and population genetic architecture of the black bean aphid, Aphis fabae. Molecular Ecology, 2011. 20(19): p. 4165-4181.

130. Pashalidou, F.G., et al., Plant volatiles induced by herbivore eggs prime defences and mediate shifts in the reproductive strategy of receiving plants. Ecology Letters, 2020. 22: p. 1-10.

131. Yip, E.C., et al., Costs of plant defense priming: exposure to volatile cues from a specialist herbivore increases short-term growth but reduces rhizome production in tall goldenrod (Solidago altissima). Bmc Plant Biology, 2019. 19.

132. Van Den Boom, C.E.M., et al., Qualitative and quantitative variation among volatile profiles induced by Tetranychus urticae feeding on plants from various families. Journal of Chemical Ecology, 2004. 30(1): p. 69-89.

133. Koch, T., et al., Differential induction of plant volatile biosynthesis in the lima bean by early and late intermediates of the octadecanoid-signaling pathway. Plant Physiology, 1999. 121(1): p. 153-162.

134. Freundlich, G. and C. Frost, Variable costs and benefits of eavesdropping a green leaf volatile on two plant species in a common garden. 2019: Biorxiv. p. 1-32.

135. Conrath, U., Molecular aspects of defence priming. Trends in Plant Science, 2011. 16(10): p. 524-531.

136. Alborn, H.T., et al., An elicitor of plant volatiles from beet armyworm oral secretion. Science, 1997. 276(5314): p. 945-949.

137. Shlichta, J.G., et al., Contrasting consequences of plant domestication for the chemical defenses of leaves and seeds in lima bean plants. Basic and Applied Ecology, 2018. 31: p. 10-20.

138. McCloud, E.S. and I.T. Baldwin, Herbivory and caterpillar regurgitants amplify the wound-induced increases in jasmonic acid but not nicotine in Nicotiana sylvestris. Planta, 1997. 203(4): p. 430-435.

139. Wu, J., et al., A Comparison of Two Nicotiana attenuata Accessions Reveals Large Differences in Signaling Induced by Oral Secretions of the Specialist Herbivore Manduca sexta. Plant Physiology, 2008. 146(3): p. 927-939.

140. Gleadow, R.M. and B.L. Moller, Cyanogenic Glycosides: Synthesis, Physiology, and Phenotypic Plasticity. Annual Review of Plant Biology, Vol 65, 2014. 65: p. 155185.

141. Ballhorn, D.J., R. Lieberei, and J.U. Ganzhorn, Plant cyanogenesis of Phaseolus lunatus and its relevance for herbivore-plant interaction: The importance of quantitative data. Journal of Chemical Ecology, 2005. 31(7): p. 1445-1473.

142. Gleadow, R., et al., Cyanogenic Glycosides, in Soil Allelochemicals, S.S. Narwal, Editor. 2011, Studium Press LLC. p. 283-310.

143. Hunter, M.D. and R.E. Forkner, Hurricane damage influences foliar polyphenolics and subsequent herbivory on surviving trees. Ecology, 1999. 80(8): p. 2676-2682.

144. Bates, D., et al., Fitting Linear Mixed-Effects Models Using Ime4. Journal of Statistical Software, 2015. 67(1): p. 1-48. 
145. Villanueva, R.A.M. and Z.J. Chen, ggplot2: Elegant Graphics for Data Analysis, 2nd edition. Measurement-Interdisciplinary Research and Perspectives, 2019. 17(3): p. 160-167.

146. Engelberth, J. and M. Engelberth, The Costs of Green Leaf Volatile-Induced Defense Priming: Temporal Diversity in Growth Responses to Mechanical Wounding and Insect Herbivory. Plants-Basel, 2019. 8(1).

147. Cofer, T.M., M. Engelberth, and J. Engelberth, Green leaf volatiles protect maize (Zea mays) seedlings against damage from cold stress. Plant Cell and Environment, 2018. 41(7): p. 1673-1682.

148. Maurya, A.K., L. Pazouki, and C.J. Frost, Plant seeds are primed by herbivoreinduced plant volatiles. 2019: Biorxiv preprint. p. 1-28.

149. Cuny, M.A.C., et al., Changes in plant growth and seed production in wild lima bean in response to herbivory are attenuated by parasitoids. Oecologia, 2018. 187(2): p. 447-457.

150. Tito, R., et al., From over to undercompensation: Variable responses to herbivory during ontogeny of a Neotropical monocarpic plant. Biotropica, 2016. 48(5): p. 608-617.

151. Focardi, S. and A. Tinelli, Herbivory in a Mediterranean forest: browsing impact and plant compensation. Acta Oecologica-International Journal of Ecology, 2005. 28(3): p. 239-247.

152. Klinkhamer, P.G.L., T. Kubo, and Y. Iwasa, Herbivores and the evolution of the semelparous perennial life-history of plants. Journal of Evolutionary Biology, 1997. 10(4): p. 529-550.

153. Schuman, M.C. and I.T. Baldwin, The Layers of Plant Responses to Insect Herbivores. Annual Review of Entomology, Vol 61, 2016. 61: p. 373-394.

154. Garcia, L.C. and M.D. Eubanks, Overcompensation for insect herbivory: a review and meta-analysis of the evidence. Ecology, 2019. 100(3).

155. Tsumele, J., D. Mlambo, and A. Sebata, Responses of three Acacia species to simulated herbivory in a semi-arid southern African savanna. African Journal of Ecology, 2007. 45(3): p. 324-326.

156. Karban, R., Tradeoff between resistance induced by volatile communication and over-topping vertical growth. Plant Signaling \& Behavior, 2017. 12(7).

157. Moreira, X., et al., Specificity of induced defenses, growth, and reproduction in lima bean (Phaseolus lunatus) in response to multispecies herbivory. American Journal of Botany, 2015. 102(8): p. 1300-1308.

158. Ballhorn, D.J., M. Heil, and R. Lieberei, Phenotypic plasticity of cyanogenesis in lima bean Phaseolus lunatus - Activity and activation of beta-glucosidase. Journal of Chemical Ecology, 2006. 32(2): p. 261-275.

159. Ballhorn, D.J., S. Kautz, and R. Lieberei, Comparing responses of generalist and specialist herbivores to various cyanogenic plant features. Entomologia Experimentalis Et Applicata, 2010. 134(3): p. 245-259.

160. Godschalx, A.L., et al., Is protection against florivory consistent with the optimal defense hypothesis? Bmc Plant Biology, 2016. 16: p. 1-9. 
161. Young, T.P., M.L. Stanton, and C.E. Christian, Effects of natural and simulated herbivory on spine lengths of Acacia drepanolobium in Kenya. Oikos, 2003. 101(1): p. 171-179.

162. Karban, R., et al., Induced plant responses and information content about risk of herbivory. Trends in Ecology \& Evolution, 1999. 14(11): p. 443-447.

163. Magrath, R.D., et al., Eavesdropping on heterospecific alarm calls: from mechanisms to consequences. Biological Reviews, 2015. 90(2): p. 560-586.

164. Pastor, V., et al., Primed plants do not forget. Environmental and Experimental Botany, 2013. 94: p. 46-56.

165. Ninkovic, V., et al., Who is my neighbor? Volatile cues in plant interactions. Plant Signaling \& Behavior, 2019. 14(9).

166. Delphia, C.M., M.C. Mescher, and C.M. De Moraes, Induction of plant volatiles by herbivores with different feeding habits and the effects of induced defenses on host-plant selection by thrips. Journal of Chemical Ecology, 2007. 33(5): p. 9971012.

167. Mithofer, A., G. Wanner, and W. Boland, Effects of feeding Spodoptera littoralis on lima bean leaves. II. Continuous mechanical wounding resembling insect feeding is sufficient to elicit herbivory-related volatile emission. Plant Physiology, 2005. 137(3): p. 1160-1168.

168. Zhang, P.J., et al., Whiteflies interfere with indirect plant defense against spider mites in Lima bean. Proceedings of the National Academy of Sciences of the United States of America, 2009. 106(50): p. 21202-21207.

169. Ponzio, C., et al., Ecological and phytohormonal aspects of plant volatile emission in response to single and dual infestations with herbivores and phytopathogens. Functional Ecology, 2013. 27(3): p. 587-598.

170. Ponzio, C., et al., Dual herbivore attack and herbivore density affect metabolic profiles of Brassica nigra leaves. Plant, Cell \& Environment, 2017: p. n/a-n/a.

171. Karban, R., S. Ishizaki, and K. Shiojiri, Long-term demographic consequences of eavesdropping for sagebrush. Journal of Ecology, 2012. 100(4): p. 932-938.

172. Ton, J., et al., Priming by airborne signals boosts direct and indirect resistance in maize. Plant Journal, 2007. 49(1): p. 16-26.

173. Kim, J. and G.W. Felton, Priming of antiherbivore defensive responses in plants. Insect Science, 2013. 20(3): p. 273-285.

174. Conrath, U., et al., Priming for Enhanced Defense, in Annual Review of Phytopathology, Vol 53, N.K. VanAlfen, Editor. 2015. p. 97-119.

175. Ali, J.G. and A.A. Agrawal, Specialist versus generalist insect herbivores and plant defense. Trends in Plant Science, 2012. 17(5): p. 293-302.

176. Bidart-Bouzat, M.G. and D. Kliebenstein, An ecological genomic approach challenging the paradigm of differential plant responses to specialist versus generalist insect herbivores. Oecologia, 2011. 167(3): p. 677-689.

177. Yip, E.C., et al., The volatile emission of a specialist herbivore alters patterns of plant defence, growth and flower production in a field population of goldenrod. Functional Ecology, 2017. 31(5): p. 1062-1070. 
178. Karban, R., P. Grof-Tisza, and J.D. Blande, CHEMOTYPIC Variation in Volatiles and Herbivory for Sagebrush. Journal of Chemical Ecology, 2016: p. 1-12.

179. Ninkovic, V., D. Markovic, and I. Dahlin, Decoding neighbour volatiles in preparation for future competition and implications for tritrophic interactions. Perspectives in Plant Ecology Evolution and Systematics, 2016. 23: p. 11-17.

180. Karban, R. and K. Shiojiri, Self-recognition affects plant communication and defense. Ecology Letters, 2009. 12(6): p. 502-506.

181. Karban, R., et al., Geographic dialects in volatile communication between sagebrush individuals. Ecology, 2016. 97(11): p. 2917-2924.

182. Kalske, A., et al., Insect Herbivory Selects for Volatile-Mediated Plant-Plant Communication. 2019: Current Biology. p. 1-10.

183. Poelman, E.H., et al., Chemical diversity in Brassica oleracea affects biodiversity of insect herbivores. Ecology, 2009. 90(7): p. 1863-1877.

184. Shlichta, J.G., G. Glauser, and B. Benrey, Variation in Cyanogenic Glycosides Across Populations of Wild Lima Beans (Phaseolus lunatus) Has No Apparent Effect on Bruchid Beetle Performance. Journal of Chemical Ecology, 2014. 40(5): p. 468-475.

185. Pardo, A., Y. Cáceres, and F. Pulido, Intraspecific variation in heritable secondary metabolites and defensive strategies in a relict tree. Journal of Plant Ecology, 2016.

186. Wetzel, W.C., N.C. Aflitto, and J.S. Thaler, Plant genotypic diversity interacts with predation risk to influence an insect herbivore across its ontogeny. Ecology, 2018. 99(10): p. 2338-2347.

187. Damestoy, T., et al., Oak genotype and phenolic compounds differently affect the performance of two insect herbivores with contrasting diet breadth. Tree Physiology, 2019. 39(4): p. 615-627.

188. Agostini-Costa, T.D., et al., Total phenolics, flavonoids, tannins and antioxidant activity of lima beans conserved in a Brazilian Genebank. Ciencia Rural, 2015. 45(2): p. 335-341.

189. Li, X.H., et al., Domestication of tomato has reduced the attraction of herbivore natural enemies to pest-damaged plants. Agricultural and Forest Entomology, 2018. 20(3): p. 390-401.

190. Raghava, T., et al., Spatial and temporal volatile organic compound response of select tomato cultivars to herbivory and mechanical injury. Plant Science, 2010. 179(5): p. 520-526.

191. Tahmasebi, Z., et al., Herbivore-induced indirect defense across bean cultivars is independent of their degree of direct resistance. Experimental and Applied Acarology, 2014. 63(2): p. 217-239.

192. Ahn, S.J., F.R. Badenes-Perez, and D.G. Heckel, A host-plant specialist, Helicoverpa assulta, is more tolerant to capsaicin from Capsicum annuum than other noctuid species. Journal of Insect Physiology, 2011. 57(9): p. 1212-1219.

193. Poelman, E.H., et al., Performance of specialist and generalist herbivores feeding on cabbage cultivars is not explained by glucosinolate profiles. Entomologia Experimentalis Et Applicata, 2008. 127(3): p. 218-228. 
194. Maurya, A.K., R.C. Patel, and C.J. Frost, Acute toxicity of the plant volatile indole depends on herbivore specialization. 2020, Springer Nature: Journal of Pest Science. p. 1107-1117.

195. Waters, D.J. and C.S. Barfield, Larval development and consumption by Anticarsia-gemmatalis (Lepidoptera, Noctuidae) fed various legume species. Environmental Entomology, 1989. 18(6): p. 1006-1010.

196. von Merey, G.E., et al., Herbivore-induced maize leaf volatiles affect attraction and feeding behavior of Spodoptera littoralis caterpillars. Frontiers in Plant Science, 2013. 4.

197. Godschalx, A.L., et al., Is protection against florivory consistent with the optimal defense hypothesis? Bmc Plant Biology, 2016. 16.

198. Mondor, E.B. and J.F. Addicott, Conspicuous extra-floral nectaries are inducible in Vicia faba. Ecology Letters, 2003. 6(6): p. 495-497.

199. Lima, P.F.S., A.L. Teixido, and E.A.S. Paiva, Herbivory-induced overcompensation and resource-dependent production of extrafloral nectaries in Luffa cylindrica (Cucurbitaceae). Acta Oecologica-International Journal of Ecology, 2018. 93: p. 16.

200. Pearse, I.S., et al., Generalising indirect defence and resistance of plants. 2020, John Wiley and Sons: Ecology Letters. p. 1-16.

201. Heil, M., Extrafloral Nectar at the Plant-InsectInterface: A Spotlight on ChemicalEcology, Phenotypic Plasticity, and FoodWebs. 2015: Annual Review of Entomology. p. 213-237.

202. Lemoine, N.P., et al., Responses of plant phenology, growth, defense, and reproduction to interactive effects of warming and insect herbivory. Ecology, 2017. 98(7): p. 1817-1828.

203. Moreira, X., et al., Plant defence responses to volatile alert signals are population-specific. Oikos, 2016. 125(7): p. 950-956.

204. Kleine, S. and C. Muller, Intraspecific plant chemical diversity and its relation to herbivory. Oecologia, 2011. 166(1): p. 175-186.

205. Younginger, B.S., et al., Is biomass a reliable estimate of plant fitness? Applications in Plant Sciences, 2017. 5(2): p. 1-8.

206. Shiojiri, K., et al., Weeding volatiles reduce leaf and seed damage to field-grown soybeans and increase seed isoflavones. Scientific Reports, 2017. 7.

207. Simpson, K.J., et al., Still armed after domestication? Impacts of domestication and agronomic selection on silicon defences in cereals. Functional Ecology, 2017. 31(11): p. 2108-2117.

208. Chinchilla-Ramirez, M., et al., Maize seedling morphology and defence hormone profiles, but not herbivory tolerance, were mediated by domestication and modern breeding. Annals of Applied Biology, 2017. 170(3): p. 315-332.

209. Andueza-Noh, R.H., J. Martinez-Castillo, and M.I. Chacon-Sanchez, Domestication of small-seeded lima bean (Phaseolus lunatus L.) landraces in Mesoamerica: evidence from microsatellite markers. Genetica, 2015. 143(6): p. 657-669. 
210. Holeski, L.M., et al., Transgenerational effects of herbivory in a group of longlived tree species: maternal damage reduces offspring allocation to resistance traits, but not growth. Journal of Ecology, 2013. 101(4): p. 1062-1073.

211. Latzel, V., et al., Adaptive transgenerational plasticity in the perennial Plantago lanceolata. Oikos, 2014. 123(1): p. 41-46.

212. Ballhorn, D.J., S. Kautz, and J.M. Laumann, Herbivore damage induces a transgenerational increase of cyanogenesis in wild lima bean (Phaseolus lunatus). Chemoecology, 2016. 26(1): p. 1-5.

213. Colicchio, J., Transgenerational effects alter plant defence and resistance in nature. Journal of Evolutionary Biology, 2017. 30(4): p. 664-680.

214. Nihranz, C.T., et al., Transgenerational impacts of herbivory and inbreeding on reproductive output in Solanum carolinense. American Journal of Botany, 2020. 107(2): p. 286-297.

215. Holeski, L.M., G. Jander, and A.A. Agrawal, Transgenerational defense induction and epigenetic inheritance in plants. Trends in Ecology \& Evolution, 2012. 27(11): p. 618-626.

216. Stassen, J.H.M., et al., The relationship between transgenerational acquired resistance and global DNA methylation in Arabidopsis. Scientific Reports, 2018. 8.

217. Latzel, V., et al., Epigenetic variation in plant responses to defence hormones. Annals of Botany, 2012. 110(7): p. 1423-1428.

218. Avramova, Z., Transcriptional 'memory' of a stress: transient chromatin and memory (epigenetic) marks at stress-response genes. Plant Journal, 2015. 83(1): p. 149-159.

219. Auge, G.A., et al., Adjusting phenotypes via within- and across-generational plasticity. New Phytologist, 2017. 216(2): p. 343-349.

220. Ding, Y., et al., Dehydration stress memory genes of Zea mays; comparison with Arabidopsis thaliana. Bmc Plant Biology, 2014. 14.

221. Song, G.C. and C.M. Ryu, Evidence for Volatile Memory in Plants: Boosting Defence Priming through the Recurrent Application of Plant Volatiles. Molecules and Cells, 2018. 41(8): p. 724-732.

222. Lin, S.M. and L.F. Galloway, Environmental context determines within- and potential between-generation consequences of herbivory. Oecologia, 2010. 163(4): p. 911-920.

223. Agrawal, A.A., Herbivory and maternal effects: Mechanisms and consequences of transgenerational induced plant resistance. Ecology, 2002. 83(12): p. 3408-3415.

224. Neylan, I.P., R. Dirzo, and M. Sobral, Cumulative effects of transgenerational induction on plant palatability to generalist and specialist herbivores. Web Ecology, 2018. 18(1): p. 41-46.

225. Groot, M.P., et al., Effects of Multi-Generational Stress Exposure and Offspring Environment on the Expression and Persistence of Transgenerational Effects in Arabidopsis thaliana. Plos One, 2016. 11(3).

226. terHorst, C.P. and J.A. Lau, Direct and indirect transgenerational effects alter plant-herbivore interactions. Evolutionary Ecology, 2012. 26(6): p. 1469-1480. 
227. Ballhorn, D.J., Constraints of Simultaneous Resistance to a Fungal Pathogen and an Insect Herbivore in Lima Bean (Phaseolus lunatus L.). Journal of Chemical Ecology, 2011. 37(2): p. 141-144.

228. Quintana-Rodriguez, E., et al., Plant volatiles cause direct, induced and associational resistance in common bean to the fungal pathogen Colletotrichum lindemuthianum. Journal of Ecology, 2015. 103(1): p. 250-260.

229. Utama, I.M.S., et al., In vitro efficacy of plant volatiles for inhibiting the growth of fruit and vegetable decay microorganisms. Journal of Agricultural and Food Chemistry, 2002. 50(22): p. 6371-6377.

230. Appel, H.M., et al., Limitations of Folin assays of foliar phenolics in ecological studies. Journal of Chemical Ecology, 2001. 27(4): p. 761-778.

231. Steets, J.A. and T.L. Ashman, Maternal effects of herbivory in Impatiens capensis. International Journal of Plant Sciences, 2010. 171(5): p. 509-518.

232. Kellenberger, R.T., et al., Trans-generational inheritance of herbivory-induced phenotypic changes in Brassica rapa. Scientific Reports, 2018. 8.

233. Nguyen, D., et al., How plants handle multiple stresses: hormonal interactions underlying responses to abiotic stress and insect herbivory. Plant Molecular Biology, 2016. 91(6): p. 727-740.

234. Ward, S.F., et al., The role of simulated spring water stress in interactions between eastern larch and larch casebearer. Arthropod-Plant Interactions, 2019. 13(4): p. 621-633.

235. Ma, X., et al., Tolerance between non-resource stress and an invader determines competition intensity and importance in an invaded estuary. Science of the Total Environment, 2020. 724.

236. Huang, W., V. Gfeller, and M. Erb, Root volatiles in plant-plant interactions II: Root volatiles alter root chemistry and plant-herbivore interactions of neighbouring plants. Plant Cell and Environment, 2019. 42(6): p. 1964-1973.

237. Kong, C.H., et al., Plant neighbor detection and allelochemical response are driven by root-secreted signaling chemicals. Nature Communications, 2018. 9.

238. van Dam, N.M. and H.J. Bouwmeester, Metabolomics in the Rhizosphere: Tapping into Belowground Chemical Communication. Trends in Plant Science, 2016. 21(3): p. 256-265.

239. Smilanich, A.M., R.M. Fincher, and L.A. Dyer, Does plant apparency matter? Thirty years of data provide limited support but reveal clear patterns of the effects of plant chemistry on herbivores. New Phytologist, 2016. 210(3): p. 10441057. 
Appendix I

Supplemental Data from Chapter II

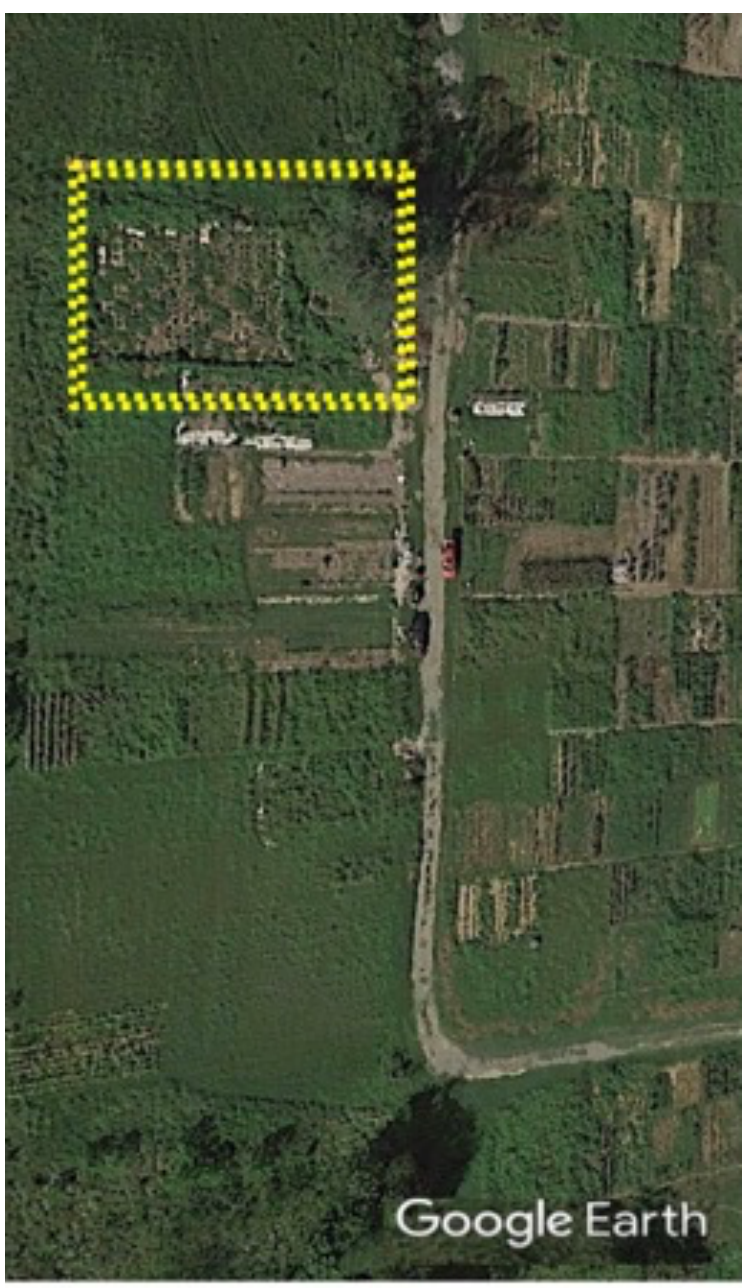

Supplemental Figure 1. Aerial view of the field site within the Blackacre Community Garden. The research area is outlined and the original picture was acquired from Google Earth. 


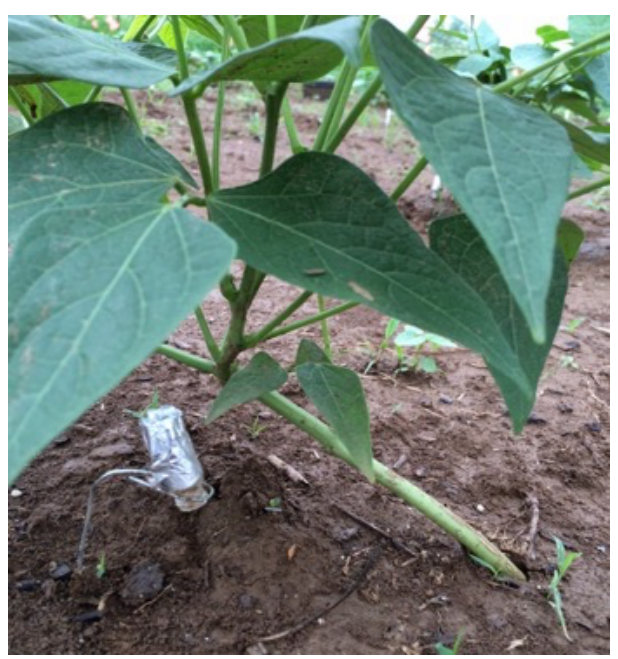

Supplemental Figure 2: Volatile administration setup. Each $2 \mathrm{~mL}$ glass vial was inverted and supported by wire stands to prevent rain water accumulation. Additionally, each vial was wrapped in aluminum foil to prevent photodegradation [75]. 


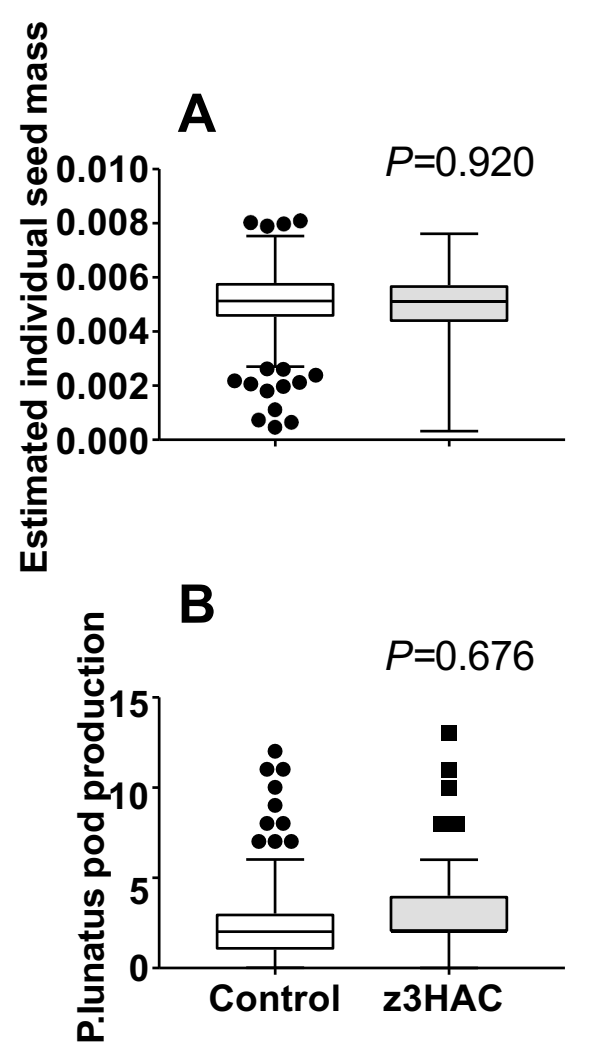

Supplemental Figure 3. Calculated individual seed mass for C.annuum (A) and total pod production for P.lunatus (B). Box plots represent the raw data ranging from the upper to the lower quartiles and the median. White boxes represent control plants; gray boxes represent plants treated with $z 3 \mathrm{HAC}$. Error bars represent the $5 \%$ and $95 \%$ of the data, and individual dots are observations that fell outside of those parameters. $P$-values represent Tukey's HSD comparisons. 

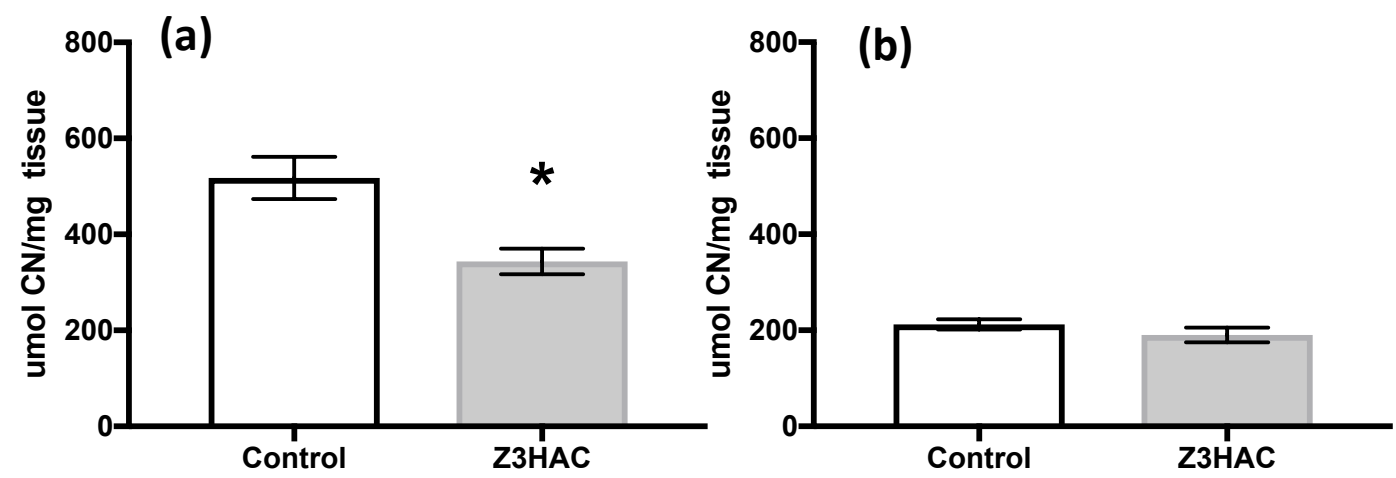

Supplemental Figure 4. Cyanide induction in Phaseolus lunatus (Lima bean) as affected

by $\mathrm{z} 3 \mathrm{HAC}$ exposure. Cyanide concentration was determined by trapping volatile $\mathrm{HCN}$ in 1.0M NaOH followed by colorometric quantification. Sink leaves $(A)$ and source leaves (B) were exposed long-term exposure of 10ng/hour z3HAC or left as a control. Points represent averages $+/$ - SE and * indicate a $p$-value $<0.05$. 


\section{Appendix II}

\section{Supplemental Data from Chapter IV}

Supplemental Table 1: Growth effects for eavesdropping on HIPVs at each timepoint.

Undamaged plants (eavesdroppers) were exposed to either A. gemmatalis HIPVS, $S$.

exigua HIPVs, or undamaged controls.

\begin{tabular}{|c|c|c|c|c|c|}
\hline & DF & Sum_sq & Mean_sq & F-value & $\operatorname{Pr}(>\mathrm{F})$ \\
\hline \multicolumn{6}{|l|}{ Bud count Day 7} \\
\hline Herbivore identity & 2 & 434.2 & 217.096 & 2.6674 & 0.07318 \\
\hline Receiver ID & 1 & 85.8 & 85.807 & 1.0543 & 0.3064 \\
\hline Congeneric vs. Heterogeneric & 1 & 7.5 & 7.492 & 0.0914 & 0.76284 \\
\hline Herbivore identity X Receiver & 2 & 142.8 & 71.379 & 0.877 & 0.41843 \\
\hline Residuals & 131 & & & & \\
\hline \multicolumn{6}{|l|}{ Bud count Day 42} \\
\hline Herbivore identity & 2 & 0.985 & 0.49248 & 3.1364 & 0.04672 \\
\hline Receiver ID & 1 & 0.354 & 0.354 & 2.2545 & 0.13564 \\
\hline Congeneric vs. Heterogeneric & 1 & 0.1006 & 0.10063 & 0.6392 & 0.4254 \\
\hline Herbivore identity X Receiver & 2 & 0.0373 & 0.01865 & 0.1188 & 0.88812 \\
\hline Residuals & 130 & & & & \\
\hline \multicolumn{6}{|l|}{ Bud count 71} \\
\hline Herbivore identity & 2 & 0.021 & 0.01048 & 0.0486 & 0.952604 \\
\hline Receiver ID & 1 & 0.2862 & 0.28619 & 1.327 & 0.251751 \\
\hline Congeneric vs. Heterogeneric & 1 & 0.0192 & 0.0192 & 0.0883 & 0.766951 \\
\hline Herbivore identity $X$ Receiver & 2 & 2.2175 & 1.10874 & 5.141 & 0.007283 \\
\hline Residuals & 113 & & & & \\
\hline \multicolumn{6}{|l|}{ Flower count day 45} \\
\hline Herbivore identity & 2 & 34 & 17.0015 & 2.6168 & 0.07685 \\
\hline Receiver ID & 1 & 14.75 & 14.7543 & 2.271 & 0.13423 \\
\hline Congeneric vs. Heterogeneric & 1 & 0.47 & 0.4715 & 0.072 & 0.78881 \\
\hline Herbivore identity X Receiver & 2 & 10.51 & 5.2539 & 0.8087 & 0.44766 \\
\hline Residuals & 130 & & & & \\
\hline \multicolumn{6}{|l|}{ Flower count day 75} \\
\hline Herbivore identity & 2 & 46 & 23.016 & 0.8244 & 0.441 \\
\hline Receiver ID & 1 & 14.8 & 14.826 & 0.5311 & 0.4676 \\
\hline Congeneric vs. Heterogeneric & 1 & 33.9 & 33.937 & 1.2188 & 0.2719 \\
\hline Herbivore identity $X$ Receiver & 2 & 24.8 & 12.405 & 0.4443 & 0.6423 \\
\hline Residuals & 115 & & & & \\
\hline \multicolumn{6}{|l|}{ Flower count day 100} \\
\hline Herbivore identity & 2 & 8.23 & 4.1173 & 0.3399 & 0.7125 \\
\hline Receiver ID & 1 & 4.44 & 4.4396 & 0.3666 & 0.5461 \\
\hline Congeneric vs. Heterogeneric & 1 & 29.62 & 29.6187 & 2.476 & 0.1184 \\
\hline Herbivore identity X Receiver & 2 & 23.88 & 11.9385 & 0.9857 & 0.3764 \\
\hline Residuals & 111 & & & & \\
\hline \multicolumn{6}{|l|}{ Pod count day 45} \\
\hline Herbivore identity & 2 & 23.7 & 11.85 & 0.853 & 0.42847 \\
\hline Receiver ID & 1 & 18.45 & 18.452 & 1.3283 & 0.25121 \\
\hline Congeneric vs. Heterogeneric & 1 & 3.5 & 3.497 & 0.2504 & 0.61765 \\
\hline Herbivore identity $\mathrm{X}$ Receiver & 2 & 70.02 & 35.009 & 2.5201 & 0.08435 \\
\hline Residuals & 130 & & & & \\
\hline \multicolumn{6}{|l|}{ Pod count Day 71} \\
\hline Herbivore identity & 2 & 30.9 & 15.455 & 0.4783 & 0.621 \\
\hline Receiver ID & 1 & 23.7 & 23.651 & 0.732 & 0.394 \\
\hline Congeneric vs. Heterogeneric & 1 & 2.3 & 2.275 & 0.0699 & 0.792 \\
\hline Herbivore identity $X$ Receiver & 2 & 99.6 & 49.823 & 1.5421 & 0.2183 \\
\hline Residuals & 116 & & & & \\
\hline \multicolumn{6}{|l|}{ Pod count Day 101} \\
\hline Herbivore identity & 2 & 708.8 & 354.41 & 1.8236 & 0.116 \\
\hline Receiver ID & 1 & 94.3 & 94.32 & 1.0543 & 0.3066 \\
\hline Congeneric vs. Heterogeneric & 1 & 0.621 & 0.31048 & 1.051 & 0.3529 \\
\hline Herbivore identity $X$ Receiver & 2 & 210.8 & 105.4 & 1.0681 & 0.347 \\
\hline Residuals & 114 & & & & \\
\hline
\end{tabular}


Supplemental Table 2: Cultivar-specific growth effects for eavesdropping on HIPVs.

Undamaged neighboring plants (eavesdroppers) that were either Fordhook or

Henderson plants were exposed to either A.gemmatalis HIPVS, S.exigua HIPVs, or

undamaged controls.

\begin{tabular}{|c|c|c|c|c|c|c|c|c|}
\hline \multirow{17}{*}{ 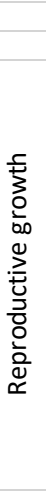 } & \multirow{3}{*}{$\begin{array}{l}\text { Response variable } \\
\text { Bud counts }\end{array}$} & \multirow{3}{*}{$\begin{array}{l}\text { Covariates } \\
\text { Herbivore Identity }\end{array}$} & \multicolumn{3}{|c|}{ Henderson } & \multicolumn{3}{|c|}{ Fordhook } \\
\hline & & & \multirow[t]{2}{*}{ DF } & F-value & \multirow{2}{*}{$\begin{array}{r}\text { p-value } \\
0.349\end{array}$} & DF & F-value & \multirow{2}{*}{$\begin{array}{r}\text { p-value } \\
0.097\end{array}$} \\
\hline & & & & 1.06546 & & 2 & 2.39435 & \\
\hline & & Time & 2 & 23.30061 & $<0.0001$ & 2 & 24.02743 & $<0.0001$ \\
\hline & & Treatment X Time & 4 & 1.14844 & 0.3393 & 4 & 0.7181 & 0.5817 \\
\hline & & Congeneric vs. Heterogeneric & 1 & 3.334 & 0.07133 & 1 & 0.4482 & 0.50495 \\
\hline & & Residuals & 86 & & & 90 & & \\
\hline & Flower counts & Herbivore Identity & 2 & 1.71911 & 0.1827 & 2 & 0.58581 & 0.5578 \\
\hline & & Time & 2 & 5.56752 & 0.0047 & 2 & 4.63057 & 0.011 \\
\hline & & Treatment X Time & 4 & 0.65149 & 0.6267 & 4 & 1.49563 & 0.2056 \\
\hline & & Congeneric vs. Heterogeneric & 1 & 1.7839 & 0.183704 & 1 & 5.6392 & 0.018678 \\
\hline & & Residuals & 150 & & & 171 & & \\
\hline & Pod counts & Herbivore Identity & 2 & 3.11302 & 0.053 & 2 & 0.32656 & 0.7223 \\
\hline & & Time & 2 & 13.35982 & $<0.0001$ & 2 & 11.76772 & $<0.0001$ \\
\hline & & Treatment X Time & 4 & 1.282 & 0.289 & 4 & 0.33864 & 0.8512 \\
\hline & & Congeneric vs. Heterogeneric & 1 & 0.2212 & 0.6402 & 1 & 1.4539 & 0.2312 \\
\hline & & Residuals & 51 & & & 87 & & \\
\hline \multirow{12}{*}{ 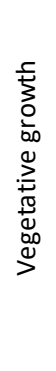 } & Leaf biomass & Herbivore Identity & 2 & 2.91 & 0.05906 & 2 & 1.7726 & 0.1751 \\
\hline & & Congeneric vs. Heterogeneric & 1 & 0.3903 & 0.5336 & 1 & 0.2844 & 0.595 \\
\hline & & Residuals & 101 & & & 101 & & \\
\hline & Stem biomass & Herbivore Identity & 2 & 0.2201 & 0.8028 & 2 & 1.3824 & 0.2557 \\
\hline & & Congeneric vs. Heterogeneric & 1 & 0.0741 & 0.786 & 1 & 0.1905 & 0.6635 \\
\hline & & Residuals & 101 & & & 101 & & \\
\hline & Root biomass & Herbivore Identity & 2 & 0.7033 & 0.498 & 2 & 2.9282 & 0.06047 \\
\hline & & Congeneric vs. Heterogeneric & 1 & 0.1213 & 0.7286 & 1 & 0.0383 & 0.8454 \\
\hline & & Residuals & 79 & & & 66 & & \\
\hline & Total biomass & Herbivore Identity & 2 & 0.5471 & 0.5803 & 2 & 1.6573 & 0.1958 \\
\hline & & Congeneric vs. Heterogeneric & 1 & & & 1 & 0.0338 & 0.8544 \\
\hline & & Residuals & 101 & & & 101 & & \\
\hline
\end{tabular}


Supplemental Table 3: Cultivar-specific growth effects for eavesdroppers on HIPVs at

each sampling date. Undamaged eavesdroppers were either exposed to A.gemmatalis

HIPVS, S.exigua HIPVs, or undamaged controls.

\begin{tabular}{|c|c|c|c|c|c|c|c|}
\hline & & \multicolumn{3}{|c|}{ Henderson } & \multicolumn{3}{|c|}{ Fordhook } \\
\hline \multicolumn{2}{|c|}{ Bud count Day 7} & DF & F-value & p-value & DF & F-value & p-value \\
\hline \multicolumn{2}{|c|}{ Herbivore identity } & 2 & 3.5773 & 0.03333 & 2 & 0.0753 & 0.92754 \\
\hline Emitter ID & & 1 & 0.1593 & 0.69101 & 1 & 3.3653 & 0.07171 \\
\hline \multicolumn{2}{|c|}{ Herbivore identity X Emitter ID } & 2 & 0.3761 & 0.68796 & 2 & 0.8381 & 0.43769 \\
\hline Residuals & & 68 & & & 58 & & \\
\hline \multirow{2}{*}{\multicolumn{8}{|c|}{ Bud count Day 42}} \\
\hline & & & & & & & \\
\hline \multicolumn{2}{|c|}{ Herbivore identity } & 2 & 1.4266 & 0.2492 & 2 & 1.2048 & 0.30563 \\
\hline Emitter ID & & 1 & 1.6177 & 0.209 & 1 & 1.4878 & 0.22648 \\
\hline \multicolumn{2}{|c|}{ Herbivore identity X Emitter ID } & 2 & 1.9857 & 0.1474 & 2 & 2.8989 & 0.06145 \\
\hline Residuals & & 53 & & & 73 & & \\
\hline \multicolumn{8}{|c|}{ Bud count 71} \\
\hline \multicolumn{2}{|c|}{ Herbivore identity } & 2 & 0.9907 & 0.3788 & 2 & 3.4947 & 0.03667 \\
\hline Emitter ID & & 1 & 0.0235 & 0.8787 & 1 & 0.074 & 0.78648 \\
\hline \multicolumn{2}{|c|}{ Herbivore identity X Emitter ID } & 2 & 1.0832 & 0.3466 & 2 & 0.076 & 0.92688 \\
\hline Residuals & & 48 & & & 60 & & \\
\hline \multicolumn{8}{|c|}{ Flower count day 45} \\
\hline \multicolumn{2}{|c|}{ Herbivore identity } & 2 & 1.9724 & 0.1494 & 2 & 1.0333 & 0.361 \\
\hline Emitter ID & & 1 & 0.0278 & 0.8683 & 1 & 1.7732 & 0.1871 \\
\hline \multicolumn{2}{|c|}{ Herbivore identity X Emitter ID } & 2 & 0.7047 & 0.4989 & 2 & 0.5313 & 0.5901 \\
\hline Residuals & & 52 & & & 73 & & \\
\hline \multicolumn{8}{|c|}{ Flower count day 75} \\
\hline \multicolumn{2}{|c|}{ Herbivore identity } & 2 & 0.6578 & 0.5225 & 2 & 3.5966 & 0.03319 \\
\hline Emitter ID & & 1 & 2.1054 & 0.15315 & 1 & 0.2641 & 0.60918 \\
\hline \multicolumn{2}{|c|}{ Herbivore identity X Emitter ID } & 2 & 3.7682 & 0.03004 & 2 & 4.2671 & 0.01843 \\
\hline Residuals & & 49 & & & 61 & & \\
\hline \multicolumn{8}{|c|}{ Flower count day 100} \\
\hline \multicolumn{2}{|c|}{ Herbivore identity } & 2 & 0.7688 & 0.4693 & 2 & 0.9852 & 0.3796 \\
\hline Emitter ID & & 1 & 0.0855 & 0.7713 & 1 & 2.7189 & 0.1047 \\
\hline \multicolumn{2}{|c|}{ Herbivore identity X Emitter ID } & 2 & 0.0784 & 0.9247 & 2 & 0.464 & 0.6311 \\
\hline Residuals & & 47 & & & 57 & & \\
\hline \multicolumn{8}{|c|}{ Pod count day 45} \\
\hline \multicolumn{2}{|c|}{ Herbivore identity } & 2 & 1.1381 & 0.3281 & 2 & 2.6079 & 0.08064 \\
\hline Emitter ID & & 1 & 1.1818 & 0.2819 & 1 & 0.0632 & 0.80226 \\
\hline Herbivore i & entity X Emitter ID & 2 & 2.1086 & 0.1315 & 2 & 0.623 & 0.53921 \\
\hline Residuals & & 53 & & & 72 & & \\
\hline Pod count $\mathrm{c}$ & y 71 & & & & & & \\
\hline Herbivore i & entity & 2 & 1.2473 & 0.29625 & 2 & 0.5928 & 0.5559 \\
\hline Emitter ID & & 1 & 0.0072 & 0.93268 & 1 & 0.0115 & 0.9151 \\
\hline Herbivore i & entity X Emitter ID & 2 & 2.9382 & 0.06235 & 2 & 1.8498 & 0.1658 \\
\hline Residuals & & 53 & & & 62 & & \\
\hline Pod count $c$ & y 101 & & & & & & \\
\hline Herbivore i & entity & 2 & 0.7712 & 0.4677 & 2 & 1.3327 & 0.2717 \\
\hline Emitter ID & & 1 & 0.0075 & 0.9315 & 1 & 1.2923 & 0.2603 \\
\hline Herbivore i & entity X Emitter ID & 2 & 0.2215 & 0.8021 & 2 & 0.5069 & 0.605 \\
\hline Residuals & & 52 & & & 58 & & \\
\hline
\end{tabular}




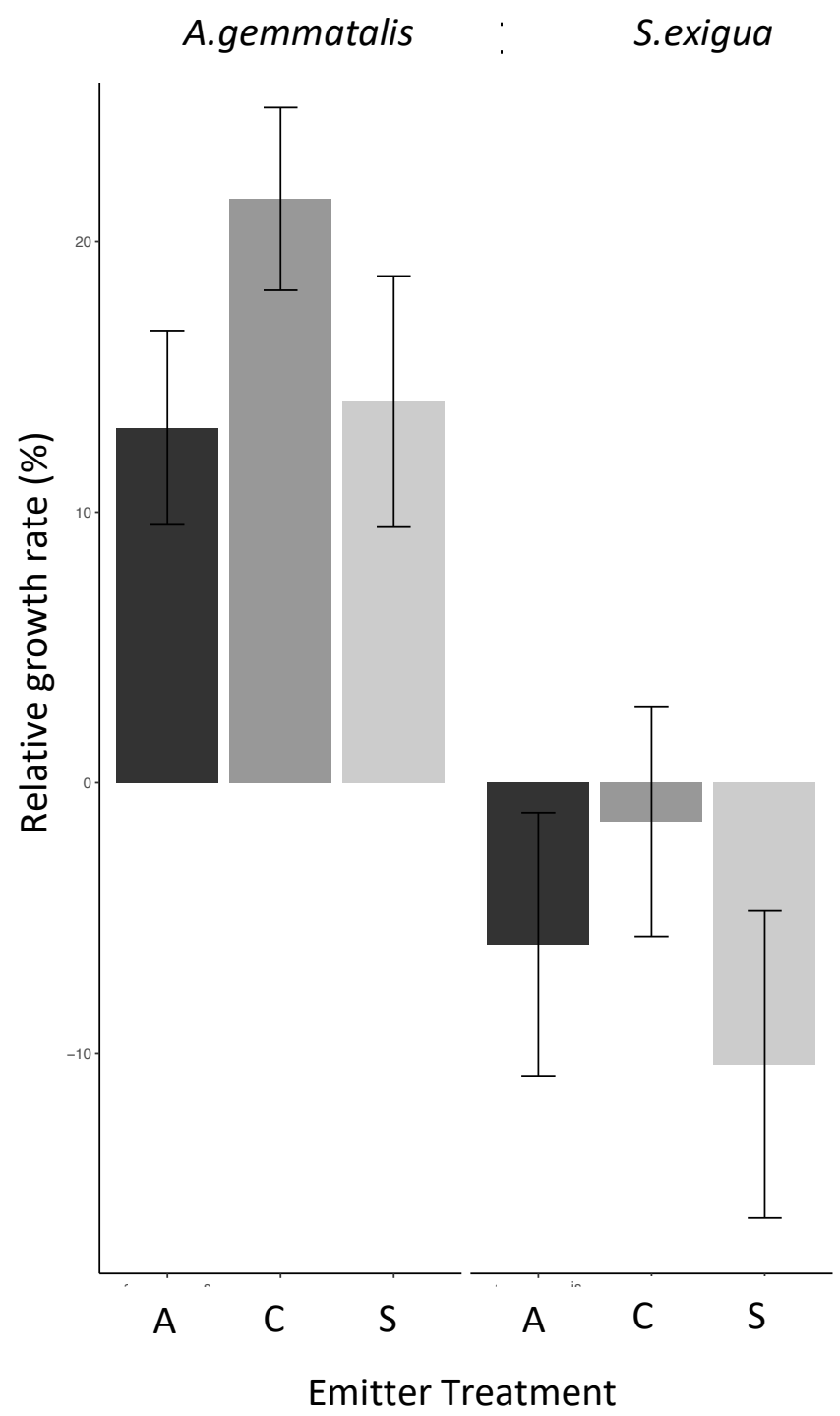

Supplemental Figure 1: Herbivore performance on neighboring eavesdroppers was unaffected by eavesdropping. Neighboring emitter plants were damaged by either A.gemmatalis or S.exigua herbivores or left as undamaged controls for 2 days before receiver plants were challenged. One mature trifoliate on each receiver plant was consequently exposed to either A.gemmatalis or S.exigua damage for 24 hours and weighed to assess relative growth rates. 


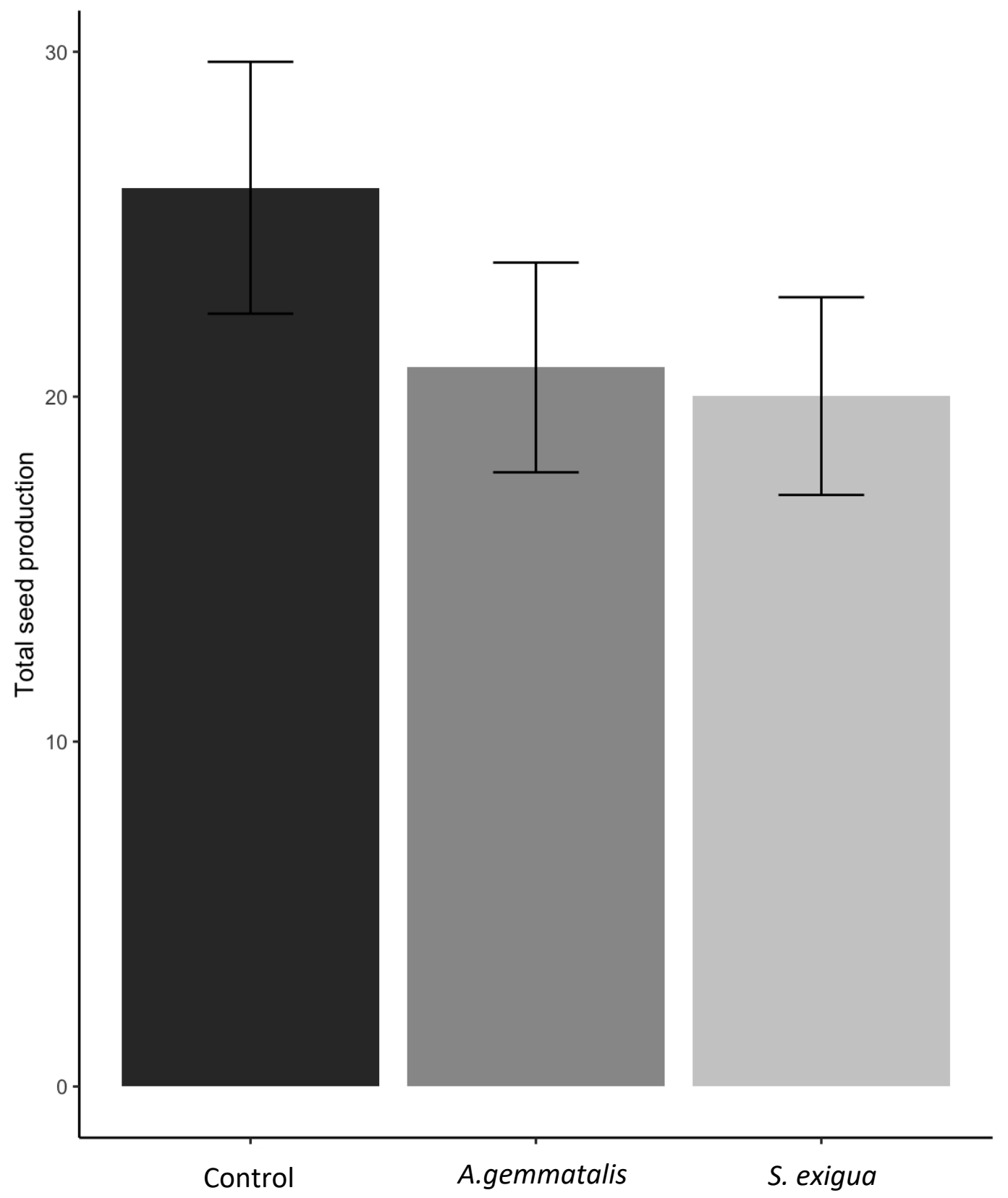

Supplemental Figure 2: Herbivore identity has no effect on seed production in eavesdropping plants. At the end of the field season, all fruits were harvested for seeds. Seeds were only counted if they had no visible herbivore damage or pathogen infection. 
Appendix III

Supplemental Data from Chapter 5

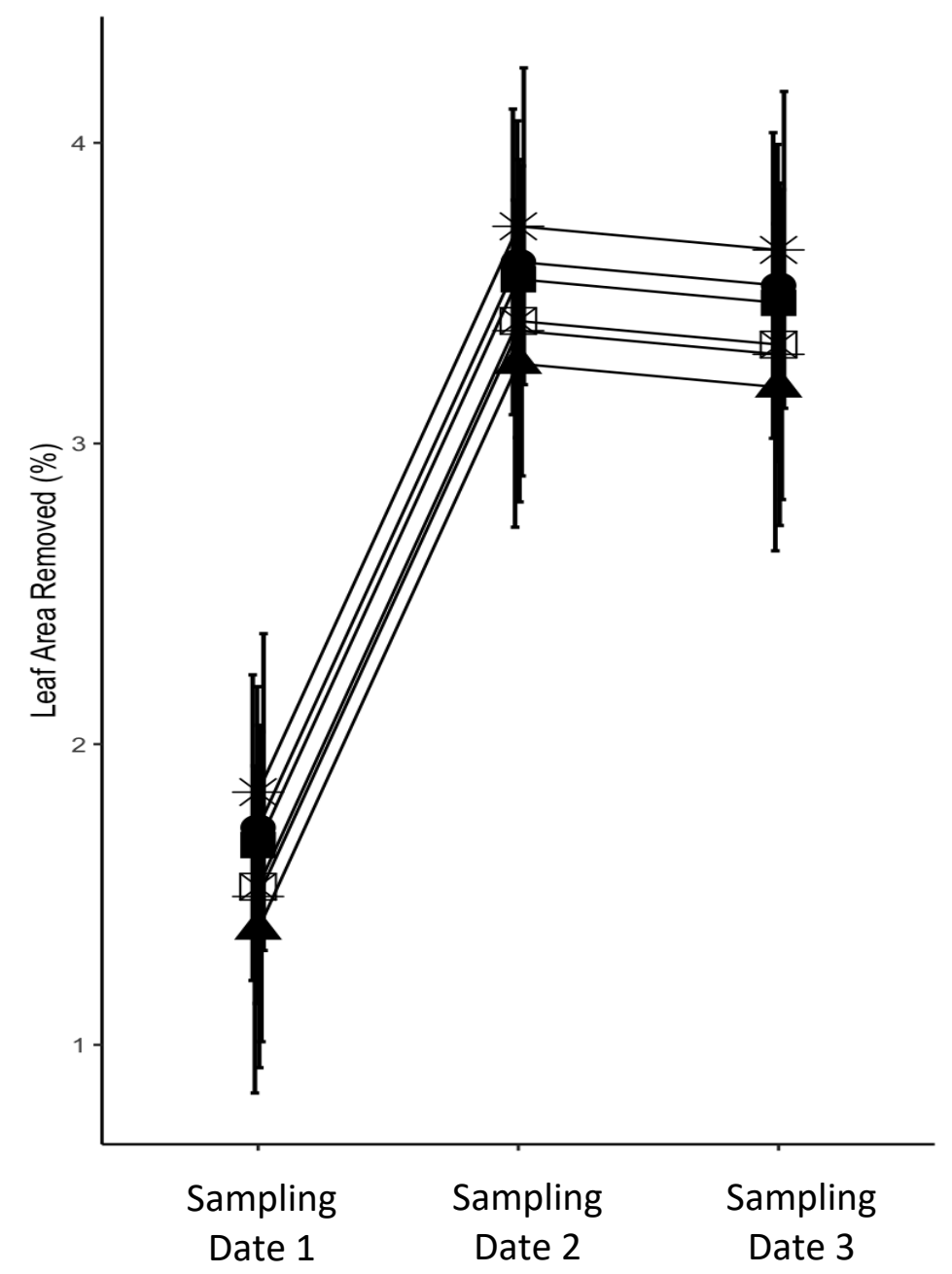

Parental Treatment

A.gemmatalisE

A.gemmatalisR

ControlE

I ControlR

$\bigotimes$ S.exiguaE

* s.exiguar

Supplemental Figure 1: Maternal effects do not impact in-field herbivore resistance via percent Leaf area removed (LAR). Values were analyzed via Two-factor ANOVA with maternal treatment and F1 simulated herbivore damage. 

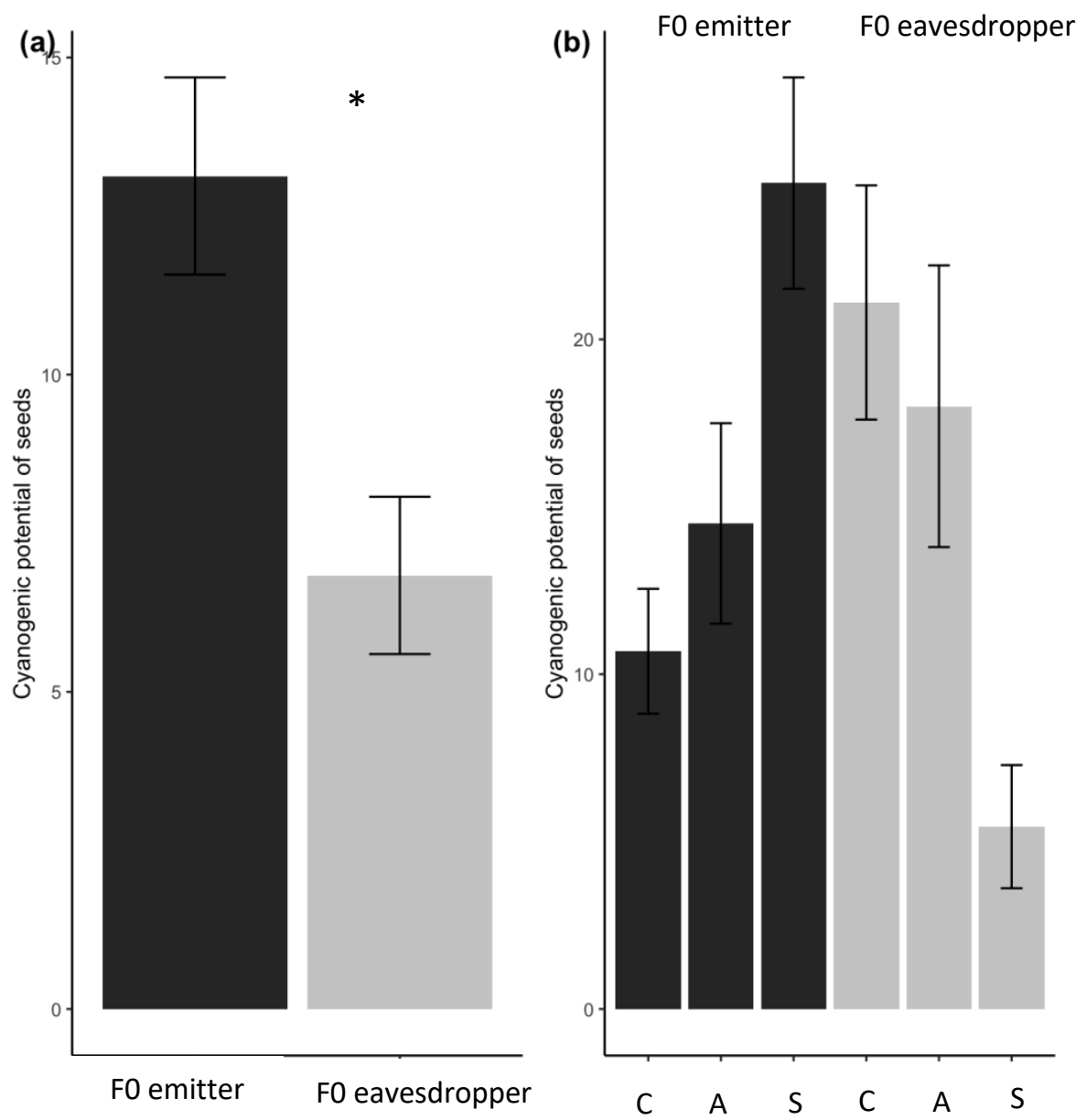

Supplemental Figure 2: Maternal effects of herbivory and HIPV-mediated eavesdropping on seed cyanide (CN) content in F0 emitters vs. receivers (a) and maternal treatment. $\mathrm{C}=$ control, $\mathrm{A}=$ A.gemmatalis treatment, and $\mathrm{S}=\mathrm{S}$. exigua treatment. F1 seeds were collected from parental plants at the end of the 2018 field season and quantified for differences in $\mathrm{CN}$ content. Asterisks $(*)$ indicate $\mathrm{p}$-values less than 0.05 after conducting a Tukey's posthoc tests. Samples sizes were relatively small $(N=10)$ and I was unable in increase the replicate size due to extenuating circumstances. 


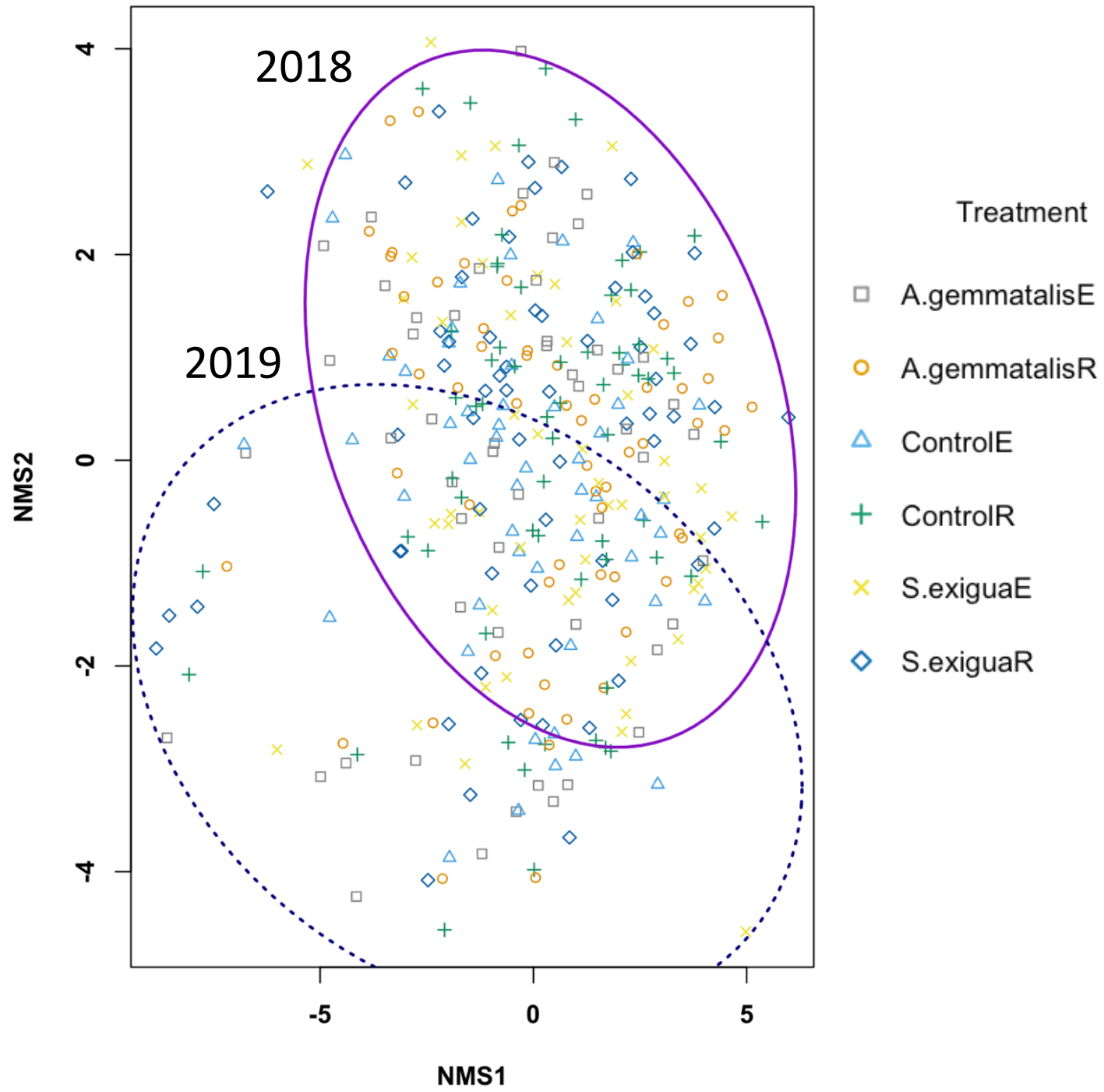

Supplemental Figure 3: Differences in overall biomass are significantly different based on growing season. MANOVA results for leaf, stem, root, and fruit biomass were conducted before visualization of differences via NMDS. 
Supplemental Table 1: Overall MANOVA results for differences in final biomass (Leaf, Stem, Root, and Fruit biomass) between F0 and F1 plants.

\begin{tabular}{|l|r|r|r|r|}
\hline Factor & df & Wilk's lambda & F & p-value \\
\hline Year & 1 & 0.3975 & 103.37 & $<\mathbf{0 . 0 0 0 1}$ \\
\hline F0_trtment & 5 & 0.9035 & 1.384 & 0.09859 \\
\hline F0_trtment x Year & 6 & 0.35117 & 13.217 & $<0.0001$ \\
\hline
\end{tabular}




\begin{tabular}{|c|c|c|c|c|}
\hline Responses & Variables & DF & F-value & p-value \\
\hline \multirow[t]{4}{*}{ Seed cyanide content } & F0 treatment & 5 & 9.4877 & 0.2414 \\
\hline & Seed ID & 71 & 1.4602 & 0.5893 \\
\hline & F0 emitter vs. Eavesdropper & 1 & 10.023 & 0.002225 \\
\hline & Residuals & 76 & & \\
\hline \multirow[t]{2}{*}{ LAR overtime } & F0 treatment & 5 & 0.1309 & 0.9853 \\
\hline & Time & 2 & 10.749 & $<0.0001$ \\
\hline
\end{tabular}


CURRICULUM VITAE

Grace Freundlich

Phone (Cell): (419) 543-2155

2301 S. Third Street Louisville, KY 40292

grace.freundlich@louisville.edu

\section{Education}

University of Louisville, KY

2016 - present

PhD Candidate in Ecology and Evolution

Dissertation Title: Costs and benefits of eavesdropping under field conditions

Dissertation Advisor: Dr. Sarah Emery

Dickinson College, PA

2010-2014

B.S. in Biology, Dickinson College

Minor: Art History; completed 11/13 courses required for the major

Overall GPA: 3.4/4.0

\section{Professional Positions:}

Graduate Research Assistant, University of Louisville

2016-present

Graduate Teaching Assistant, University of Louisville

2016-2017; 2019

NSF Graduate Research Assistant, University of Louisville

2017-2018

Research Assistant II, Ohio State OARDC

2014-2016

Undergraduate Research Assistant, Dickinson College

2013-2014 


\section{Publications:}

*denotes mentored undergraduate

Arnold T, Freundlich G, Weilnau T, Verdi A, Tibbetts IR (2014) Impacts of Groundwater Discharge at Myora Springs (North Stradbroke Island, Australia) on the Phenolic Metabolism of Eelgrass, Zostera muelleri, and Grazing by the Juvenile Rabbitfish, Siganus fuscescens. PLoS ONE 9(8): e104738.

Bissmeyer S*, Freundlich G, and Frost C (2018) The Influence of Dose of a Plant-Derived Volatile Cue on Arabidopsis thaliana Resistance against Insect Herbivores, Kentucky Journal of Undergraduate Scholarship: Vol. 2: Issue 1, Article 10.

\section{Publications in preparation (* denotes mentored undergraduate):}

Freundlich G. and Frost C (2019) Costs and benefits of eavesdropping on cis-3-hexenyl acetate on two plant species in a common garden. Biorxiv preprint https://www.biorxiv.org/content/10.1101/370692v2

Freundlich G, Greenberg L ${ }^{*}$, Bissmeyer S*, Nunamaker V*, and Frost CJ. cis-3-hexenyl acetate exposure differentially affects growth-defense tradeoffs.

Freundlich G, Pickett $\mathrm{C}^{*}$, Shields $\mathrm{M}^{*}$, Wong V*, and Frost CJ. Herbivore identity significantly impacts neighboring fitness effects in Phaeseolus lunatus under field conditions.

Freundlich G, Frost C, and Emery S. Transgenerational effects of eavesdropping are not observed in response to HIPVs under field conditinos 
Van Neerbos, Frank, Freundlich G, Yang C*, Curry K*, van Loon J, Eason R, and Pazouki L. Transgenerational herbivory impacts tri-trophic interactions in early plant development.

Awards:

Plant-Herbivore Interactions Gordon Research Seminar invited speaker $\quad 2019$

NSF GRFP Honorable mention $\quad 2017$

Kentucky Academy of Sciences Oral presentation in Ecology and Environmental

Science; Second place

Awarded Grants:

University of Louisville Dissertation Completion Grant \$5,000 2020

University of Louisville GNAS research award \$250 2019

Biology Graduate Student Association Research grant \$150 2019

University of Louisville A\&S travel award \$350 2019

$\begin{array}{ll}\text { University of Louisville GNAS travel grant \$350 } & 2019\end{array}$

UofL Biology graduate student presentation award \$1000 2018

University of Louisville A\&S creative grant \$500 2017

\section{Presentations:}

*denotes undergraduate co-presenter

Freundlich, G and Frost CJ, 2019 Is there a cost to "smelling" danger? cis-3-hexenyl acetate mediated defense priming is independent of fitness costs, Ecological Society of America, Louisville

Freundlich G and Frost CJ, 2019 The cost of "smelling" danger: source and duration of HIPVs differentially affect growth and reproductive costs in Phaseolus lunatus, Gordon Plant Herbivore Interactions, Oral presentation at seminar, poster at the conference, Ventura, CA 
Freundlich $\mathbf{G}$ and Frost CJ, 2018 Exposure to the green leaf volatile cis-3-hexenyl acetate differentially influences growth and fitness of Phaseoulus lunatus and Capsicum annuum under field conditions, Ecological Society of America, New Orleans, LA

Freundlich G and Frost CJ, 2018 The green leaf volatile cis-3-hexenyl acetate differentially influences fitness of field-grown Phaseoulus lunatus and Capsicum annuum, Midwestern Ecology and Evolution Conference, Kellogg Biological Field Station, $\mathrm{MI}$

Shields $\mathrm{M}^{*}$, Freundlich G, and Frost CJ, 2018 Determining the Cyanogenic Potential of Two Land Races of Lima Bean, Phaseolus lunatus, Kentucky Academy of Sciences, Berea College, KY

Freundlich G 2017 Differential fitness occurs in response to varying duration periods of the herbivore-associated cue, cis-3- hexenyl acetate, Kentucky Academy of Sciences, Berea College, KY

Freundlich G 2017 Species-Specific Responses to Cis-3 hexenyl acetate Result in Differential Fitness Costs within Phaseoulus lunatus and Capsicum annuum Oral Presentation; UofL Graduate Student Research Conference, University of Louisville

Freundlich G and Frost CJ 2017 Plant Derived Volatiles Alter Fitness in a Species-Specific Manner Oral Presentation, Kentucky Academy of Sciences, Murray State, KY

Freundlich G 2016 On High Alert: Differential Fitness Costs Upon Continuous Exposure to the Priming Stimulus Cis-3-Hexenyl Acetate Poster Presentation; Kentucky Academy of Science, University of Louisville, KY 


\section{Teaching Experience:}

Instructor of Record for Chemical Ecology 435/635

2019

Graduate Teaching Assistant at the University of Louisville

2016-2018

Teaching Assistant for Biology 121: This is Your Life

2014

Teaching Assistant for Biology 318: Animal Development

2013

\section{Undergraduate and post-bac mentees:}

Allie Peot: Investigating species-specific tradeoffs

2016-2017

Sarah Bissmeyer: Dose of a volatile cue effects on Priming

2016-2017

Liana Greenburg: Volatile duration effects on plant-pollinator interactions

2017

Gabe Joachim: Drought and salinity affect recovery in Mimosa pudica

2017

Carly Nunamaker: Volatile duration effects on growth-defense tradeoffs

2017

Connor Slone: Transgenerational effects of herbivory on germination

2017-2019

Vicki Wong: Herbivore identity affects growth-defense tradeoffs

2018

Courtney Pickett: Herbivore identity affects growth-defense tradeoffs.

2018

Maria Shields: Cyanogenic potential is affected by herbivore identity

2017- 2019

Myron Adams: F0 effects of herbivory on F1 growth and defense profiles

2019

Emma Isson: Parental herbivory affects defense induction in lima bean

2019

\section{Public Outreach and Departmental Service:}

Graduate student representative for Quantitative Ecologist search

2019-present

Community outreach at Blackacre Conservancy

2017-present

Biology Graduate Student Organization Graduate Committee chair

2019-present

Gallatin county scientific literacy outreach coordinator

2018

Kentucky Academy of Sciences Middle School Mentor

2018 
Biology Graduate Student Organization Vice President and Treasurer

Biology Graduate Student Organization Secretary

2017-2018

Scientific Outreach Field Organizer for Lexington Local Schools

2015

Professional Development:

University of Louisville Graduate Teaching Academy 1\&2

2019-present

University of Louisville Grant Writing Workshop

2017

\section{Professional Society memberships:}

Ecological Society of America

2018-present

Sigma Xi Honor Society

2018-present

Kentucky Academy of Sciences

2016-present 